\title{
Disconnections and Other Defects associated with Twin Interfaces
}

\author{
J. P. Hirth ${ }^{\mathrm{a}}$, J. Wang ${ }^{\mathrm{b}, *}$, C. N. Toméc \\ ${ }^{\text {a }}$ MPA-CINT, Los Alamos National Laboratory, Los Alamos, NM 87545, USA \\ ${ }^{\mathrm{b}}$ Mechanical and Materials Engineering, University of Nebraska-Lincoln, Lincoln, NE \\ 68588, USA \\ ${ }^{c}$ MST-8, Los Alamos National Laboratory, Los Alamos, NM 87545, USA \\ * Corresponding author: Jian Wang (jianwang@unl.edu)
}

\begin{abstract}
The general topological model for interfacial defects is reviewed and expanded, and the role of these defects in the coupled shear - migration of interfaces is explored. We focus on twinning in hexagonal metals for many defect examples. The definition of shuffles within the topological model is presented. The concept of partitioning of the rotational component of elastic distortions at a grain boundary or interphase interface has recently been elucidated. This work shows that rotational coherency has an important role in twinning. The role of disconnections in type II twins is presented.
\end{abstract}

Keywords: interfacial defects, twinning, hexagonal metals

\section{Index}

1. Introduction

2. Interface types and the Topological Model

2.1 Interface types

2.2 The Topological Model

2.3 The Gibbs interface

3. Interfacial Line-Defects

3.1 Defect character

3.2 Terrace dislocations and disconnections

3.3 Terrace disclinations

3.4 Junction defects

3.5 Multiple height twinning dislocations

4. Motion of unit disconnections

4.1 Preliminary remarks

4.2 Exchange cells 


\subsection{Effects associated with $p$}

4.4 Thermodynamic driving force

4.5 The P/B interface

4.6 Arrays and motion of disconnections

4.7 Combination of disconnections

5. Facets and disclinations

6. Twin nucleation

6.1 Homogeneous nucleation

6.2 Heterogeneous nucleation

7. Synchroshear

8. Interaction of matrix dislocation and twin terrace

9. Incoherent boundaries and the plane of shear

10. Twin intersections and duplex twinning

11. Type II twinning

12. Multiscale modelling of deformation twinning in $h c p$ metals

12.1 Plastic deformation in $h c p$

12.2 Modelling twinning in crystal plasticity models

12.3 Modelling nucleation and propagation of twins

12.4 Models incorporating statistical variation

13. Discussion

14. Summary

Acknowledgments

References

Appendix A. The Pond topological theory of bicrystal crystallography

Appendix B. Topological analysis of single defects

B.1 Burgers vector

B.2 Steps

B.3 Imperfect and domain defects

B.4 Shuffles

B.5 Other defects.

Appendix C. Twin notation

Appendix D. Arrays of defects

Appendix E. List of abbreviations 


\section{Introduction}

The principal objective of the present work is to discuss the structure of interfaces and mechanisms of interface motion within the Topological Model (TM) [1-5]. A list of such commonly used acronyms is given in Appendix E. The basis for the TM is the topological theory of that describes the crystallography of bicrystal interfaces in terms of dichromatic patterns $[1,2]$. Here, we do not emphasize formal crystallography, briefly reviewed in Appendix A, but emphasize physical interpretations in the TM, developed in terms of circuits in Appendix B. We concentrate on interfaces which exhibit terrace defect structures: coherent terraces have relatively low interfacial free energy, and any coherency strains arising are removed at long-range by superposed arrays of line-defects $[6,7]$. Under the constraint that coherency stresses are imposed on both crystals, the coherent terrace would be a singular interface corresponding to a cusp in a Gibbs-Wulff plot of interfacial free energy as a function of interface orientation. The presence of such a cusp is the key underlying criterion for the presence of terrace structures. The defects that relax the coherency strains are designated interfacial dislocations, or disconnections if they also exhibit step character [6,8]. Line defects with disclination character [9-13] can also be present, for example at junctions between interface facets [1]. The disclination structures, recently studied [14-16], have received little attention and are emphasized here. Interfacial dislocations have an important function in accommodating misfit at static interfaces, and in this role are typically referred to as "misfit" dislocations [17-18]. Disconnections can also fulfil this role. However, an additional function of disconnections is that motion along an interface produces both plastic deformation and interface migration simultaneously. The latter two functions are implicit in the mechanistic interpretation [19-22] of the Phenomenological Theory of Martensitic Crystallography (PTMC) [23-25], and the dual dislocation-step nature of the defects was qualitatively considered by several groups, as reviewed in [3]. The two functions were formally defined in the TM $[4,5]$, see in particular Appendix A. Consequently, the TM is key in explaining displacive phase transformations and coupled shear-migration at grain boundaries [26]. Twinning is an important example of coupled motion [27-31], and, in this article, for examples, we focus on twinning in hexagonal close-packed ( $h c p$ ) metals 
where twinning is a major deformation mode [32], although other interfaces are also mentioned. Additional extended twin defects with pure step character are also considered.

Twinning disconnections or twinning dislocations (TDs) and the role of shuffling in twinning are considered in the review by Christian and Mahajan [32]. The formal phenomenology of twins has also been reviewed recently in [33], as have the mechanisms of twinning in nanostructures [34]. With these as foundations, we present a formal definition of dislocations and disconnection in terms of the TM, including relaxation effects near the twin interfaces. An important distinction for twin is that, unlike more general martensitic transformations, there is no misfit on twin terrace planes. Therefore some structures, driven by relief of coherency, are not present for the twinning case.

The emphasis on observations is for deformation twins (generated by mechanical loading). However, the models and topography apply also for annealing twins. The distinction is the rate of growth, i.e., the velocity of TDs. For deformation twins, the growth rate is high and kink velocities on a TD line are large, athermal, and damping controlled. For annealing twins (induced by heat treatment, recrystallization), the growth rate is small, and kink velocities are small and thermally activated. There is a parallel for martensite, with fast, athermal growth as for steels, and slow thermally activated growth as for shape-memory alloys. The present work considers structural aspects of twins. The details for kink motion are considered briefly for disconnections in [3]. The details are directly analogous to those for the motion of kinks in dislocation glide, treated extensively in standard texts on dislocation theory.

In Section 2, distinctive types of terrace-defect interfaces are identified within the broader spectrum of interphase and grain boundaries. The TM, based on the dichromatic symmetry of bicrystals $[1,35]$, is introduced for cases of interest here. Commensurate/coherent dichromatic patterns (CDP) and complexes (CDC) are considered. An account of the character of interfacial defects follows in Section 3: here, we distinguish the categories of defects: dislocations, disconnections, disclinations and pure steps, identifying the types of interfaces in which they arise. Section 4 focuses on the character of active disconnections, particularly in twinning, and their role in twin growth, and introduces the exchange cell. Disconnection motion can be conservative or 
may require long-range diffusion. Short-range atomic motions, designated shuffles, which do not produce plastic strains, generally also accompany disconnection motion. In $h c p$ twinning, experimental evidence is accumulating showing that disconnection motion is important not only along coherent twin boundaries, but also in other coherent facets observed to interrupt coherent twins [36-37]. In section 5 we demonstrate that such facets can be produced on a twin boundary by a dual process. First, unit disconnections combine to form a large step. Second, the step rotates to form a coherent face with the concomitant formation of disclinations at facet-twin intersections [1]. We also show that the distortion fields of such junction disclinations can be locked when a twin meets its conjugate. In another mechanism, serrated facet configurations could result by operation of interfacial sources of unit disconnections [38]. Moreover, these mechanisms accommodate continued twin growth. As we describe in Section 6, facets can have a low free energy, and may play a significant role in twin nucleation. Section 7 describes synchroshear, produced by a special type of dislocation. Section 8 describes the interaction of extrinsic dislocations with twin boundaries, leading to cutting or dissociation in the boundary. Recent studies $[39,40]$ have shown that disconnections formed in such processes are significant sources of twin growth and interface multiplication mechanisms. Section 9 treats incoherent twin boundaries and boundaries orthogonal to the twinning direction and twin plane normal. Twin intersections and duplex twinning are considered in Section 10. Type II twinning is treated in Section 11. Section 12 deals with macroscopic effects: accommodation and constitutive behaviour. Discussion in Section 13 and a summary follow.

Throughout the text, we emphasize mechanisms of interface motion, and relegate crystallographic and other details to Appendices. The TD Burgers vectors and shear offsets are consistent with the descriptions in [32-34]. New descriptions of shuffling, disclinations, and type II twinning are provided.

\section{Interface types and the Topological Model}

2.1 Interface types 
We are interested in unique interfaces that can comprise a terrace plane. Hence we do not treat Vernier interfaces as can occur in liquid crystals or in most incoherent interfaces. The exception is a high-angle pure tilt wall. The broadest categorisation of interfaces entails two subcategories:

I. Interphase boundaries, where there is a transition of either the crystal structure or the composition, or both,

II. Grain boundaries, where only a change of crystal orientation occurs.

Terrace-defect interfaces arise in both categories. For example, in the first category, epitaxial interfaces [41] and martensitic interfaces [42] are important. In both cases, the terraces generally require biaxial strains in order to become commensurate. In the simplest case, there are matching primitive translation vectors (or crystallographic translation vectors) in two independent directions parallel to the terrace planes: this is the coherent or commensurate case. Otherwise, in the more general commensurate case, there is matching of integral multiples of primitive translation vectors, also known as domain epitaxy [43]. Figure 1(a) shows an example of misfit dislocations accommodating the coherency strains present at a semi-coherent hetero-epitaxial interface [44].

Recent studies of martensitic transformations show that these interfaces exhibit commensurate terraces [20-22, 45-48]. There is a transition of crystal structure across the interface, but not of composition. The TM demonstrates that such terraced interfaces, with coherency strains accommodated by arrays of dislocations and disconnections, can migrate without long-range diffusion [49]. An example is illustrated in Figure 1(b) [50]. In that work on zirconia, the offsets on the terrace were shown to also have dislocation character, i.e. they are TDs. Motion of such disconnections along the interface produces both the transformation deformation and the interface motion. Some diffusive transformations [51] are closely related to martensitic transformations. Here, the crystallographic forms of transformation products resemble martensites, but long-range diffusion also occurs in order to accommodate composition change. This is accounted for in the TM because disconnection motion conserves atomic sites, as in the martensite case, 
but, unlike the martensite case, these sites may become occupied by different chemical species during the transformation [3].
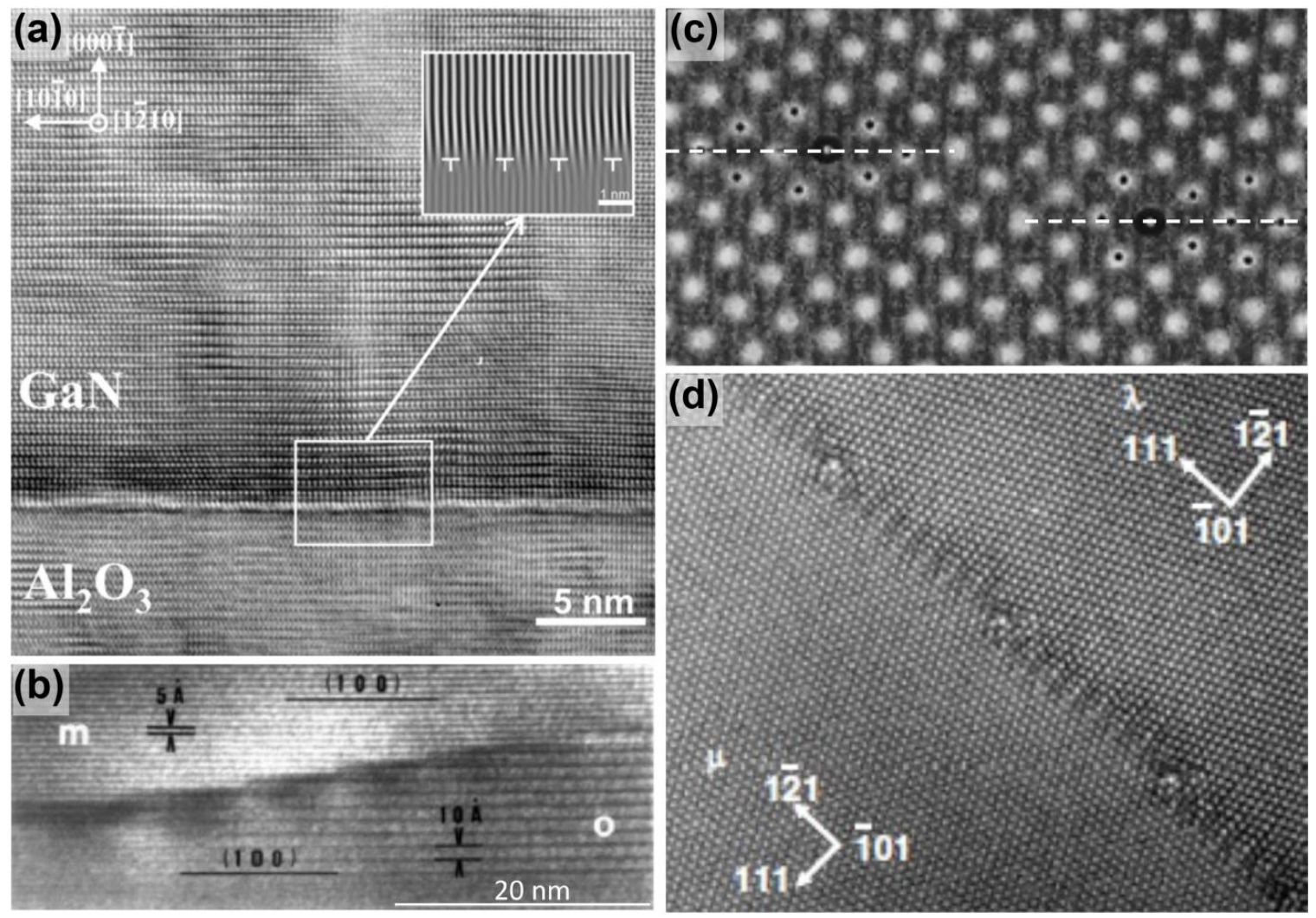

Figure 1. TEM observations of terrace-defect interfaces. (a) Semi-coherent hetero-epitaxial interface [46], (b) Bright field image of orthorhombic-monoclinic martensitic transformation interface in $\mathrm{ZrO}$, showing an array of disconnections [50]. (c) (1012)) twin containing a twinning disconnection in Ti, and (d) Bright field TEM image showing what the authors describe as "a $<110>/ 90^{\circ}$ boundary in Au showing a segment of coherent boundary with three superimposed disconnections" [53].

Historically, grain boundaries have often been modelled as arrays of dislocations [52]. The separation of individual dislocations in low-angle boundaries, or of disconnections on a terraced interface with a low angle of tilt are sufficient for regions of distorted single crystal to be discerned, as shown in Figure 1(c) as well as in the work on Au in Figure 1(d) [53]. Such regions can be regarded as naturally commensurate terraces. In the high-angle regime, the cores of the component dislocations overlap [54], and alternative descriptions of their structures are helpful. Thus, the structure of twin 
boundaries can be regarded as naturally coherent terraces: an example is illustrated in Figure 1(c). In the classical notation for the macroscopic crystallography of twins [32, 55], this is a (10 $\overline{1} 2)$ twin in $\mathrm{Ti}$, i.e., where the adjacent crystals are interrelated by a mirror plane parallel to the $\boldsymbol{K}_{1}$ plane and a diad axis parallel to the $\boldsymbol{\eta}_{1}$ twinning direction. Twins with rational values of $\boldsymbol{K}_{1}$ and $\boldsymbol{\eta}_{2}$ are designated Type I, and those with rational values of $\boldsymbol{K}_{2}$ and $\boldsymbol{\eta}_{1}$ are Type II: all four parameters have rational indices of the same variant for compound twins. The twinning elements $\boldsymbol{K}_{1}, \boldsymbol{K}_{2}, \boldsymbol{\eta}_{1}, \boldsymbol{\eta}_{2}$ define the macroscopic relationship between the crystals as summarized in Appendix C. These can also be represented additional microscopic parameters that characterise the interface structure. These can also be represented by symmetry operations, as summarised in Appendix A.

\subsection{The Topological Model}

The application of the TM to interphase interface and grain boundary structures is mentioned above. The TM is based on the dichromatic symmetry analysis of bicrystals $[1,35]$, summarized in Appendix A, wherein one crystal, conventionally the matrix, is regarded as white, designated $\lambda$, and the other, conventionally the product, as black, $\mu$. These designations are used interchangeably when we connect formally to the topological theory [1]. In some figures it is more convenient to use other colours for sites in the two crystals. Thus, for twins, the crystals are interrelated by colour-reversing mirrors and/or diads (also known as anti-mirrors and anti-diads [35]), while interphase interfaces cannot exhibit such symmetries [1]. On the other hand, both interphase interfaces and grain boundaries can exhibit common symmetry, for example where mirror planes in the two crystals are parallel.

In addition to real bicrystals, as revealed by transmission electron microscopy (TEM) or by atomistic simulation, four constructions are helpful for visualising crystallographic features such as facet orientations, Burgers vectors and disclination rotations: these are dichromatic patterns and complexes, ideal bicrystals and exchange cells (e-cells). The dichromatic constructions also serve as perfect reference lattices for circuit operations. Below, we outline these features for three interfaces that include 
aspects covered subsequently in some detail, providing a connection to much past work utilizing the TM.

The first interfaces of interest are (1012) twins in $h c p$ metals [38-40], such as the Ti boundary shown in Figure 1(c). For the present study of coherent terraces separated by defects, the important pattern is the commensurate dichromatic pattern (CDP), which has a corresponding commensurate dichromatic complex (CDC). Formally, the CDP is the union of the space groups of the $\lambda$ and $\mu$ lattices, relatively rotated so that there is a unique coincident plane, and is depicted graphically in Figure 2(a). The $\lambda$ lattice is represented by open symbols, and $\mu$ by filled ones, and their unit cells are indicated in the figure. The conventional notation in the TM is that the $y$-axis is normal to the interface and the $\mathrm{x}$-axis points toward the reader. The $\lambda$ and $\mu$ lattices are interrelated by the transformation matrix $(\boldsymbol{P}, \boldsymbol{p})$, where $\boldsymbol{P}$ represents reflection in the $\boldsymbol{K}_{1}$ plane, and $\boldsymbol{p}$ is a rigid displacement of the $\mu$ lattice with respect to that of $\lambda$. Then, the coordinate transformation of a $\mu$-vector, $\boldsymbol{w}(\mu)$, is $\boldsymbol{w}(\lambda)=\boldsymbol{P} \boldsymbol{w}(\mu)+\boldsymbol{p}$. We note that $\boldsymbol{P}$ can be alternatively expressed, e.g. by a rotation of $180^{\circ}$ about $\boldsymbol{\eta}_{1}$, or, as is particularly relevant in this work, by a rotation of $\theta$ about the projection direction of Figure 2(a), [1 $12 \overline{1} 0]$ : in the case of $\mathrm{Mg}$ for example, $\theta=87.3^{\circ}$. The aim of the topological theory [1] is to begin with constructions of highest symmetry and then consider successive symmetry breaking in creating real structures. Hence, we define the initial reference position of the lattices so that $\boldsymbol{p}=0$, meaning that the chosen origins of the $\lambda$ and $\mu$ lattices coincide along the coincident plane of Figure 2(a): for $h c p$, the convenient origins are centers of symmetry, designated COS sites. The lattice of COS sites alone comprises the CDP. For this choice, the CDP exhibits maximal point symmetry, $2 / \mathrm{mm}^{\prime} \mathrm{m}^{\prime}$ (the formal notation and procedure for characterizing symmetry is summarised in $[1,35])$. In Figure 2(a) the horizontal dashed line represents an anti-mirror planes, designated $m^{\prime}$. That is the plane would be a mirror plane $\mathrm{m}$ were $\mu$ and $\lambda$ the same colour but the plane becomes an anti-mirror plane when the colour distinction is imposed. In most prior work the focus was on determining $\boldsymbol{b}$ and the step height $h$. The CDP lattice need not be fixed on lattice sites: whether it is or not, it suffices to determine $\boldsymbol{b}$ and $h$. Here, we are interested in further details of the interface structure represented by the CDC. 

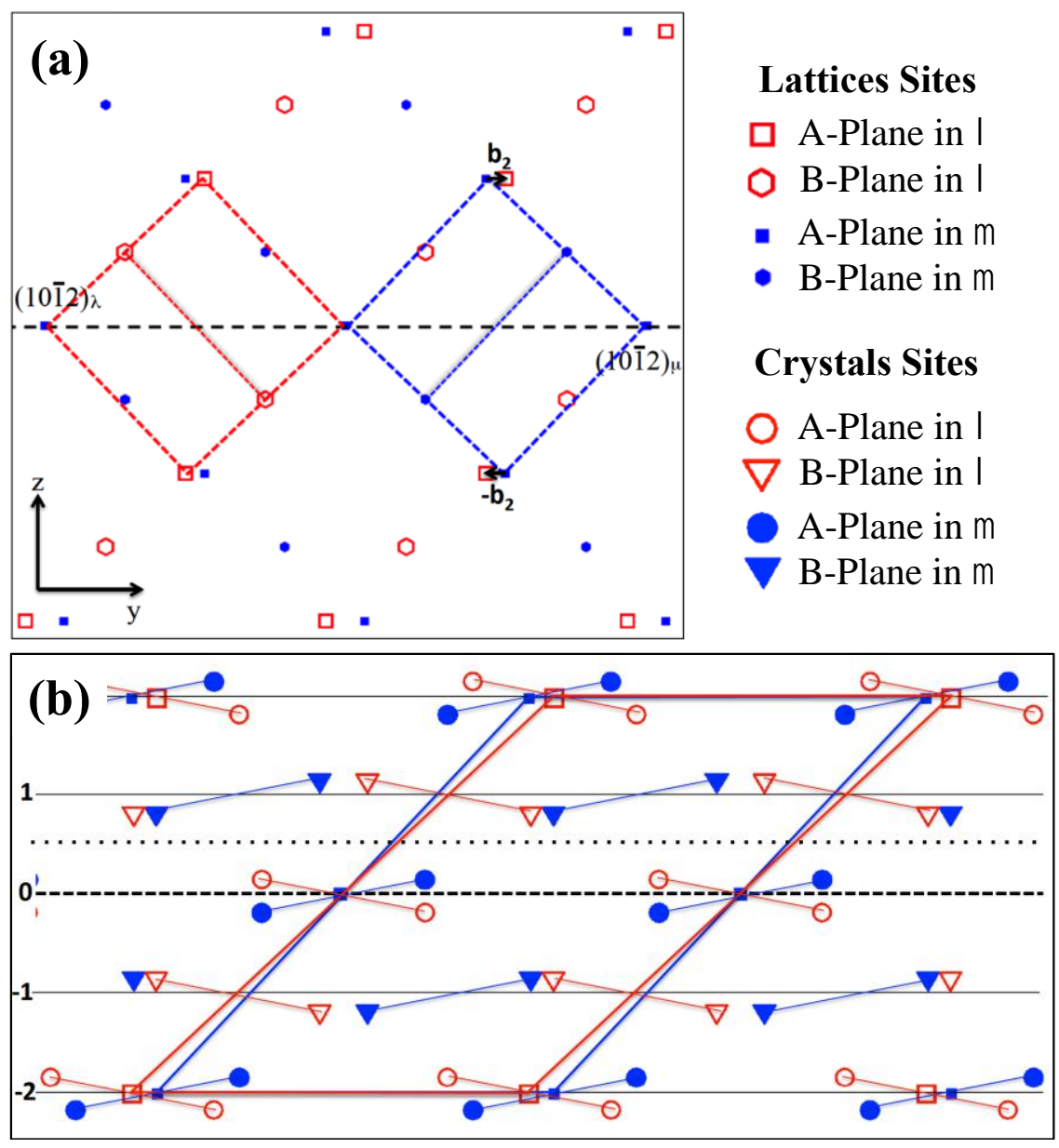

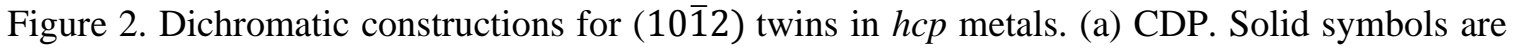
$\mu$ COS sites, open ones $\lambda$. (b) CDC. Basis pairs connected by thin dashed lines, dividing surface indicated by heavy dashed line, commensurate plane by dotted line.

The CDC is constructed, exhibiting all atomic sites of the $\lambda$ and $\mu$ crystals. With the atomic site origin, the CDP is identical to the CDC. The CDC is then a perfect reference lattice exhibiting high symmetry. The origin is selected as a coincident atom site, or, if there is a basis, the center-of-symmetry or COS site, in the $\lambda$ crystal adjoining the interface (Appendix B). Ideal reference bicrystals can be created from a CDC by selecting a notional dividing surface, as shown in Figure 2(b), and populating $\lambda$ atomic sites above this plane and $\mu$ sites below [1]. This surface identifies the interface (terrace) plane in the ideal bicrystal. In order to avoid an ambiguity, the product crystal plane adjoining the dividing surface is identified with the white crystal and the dividing plane is then selected as lying between this white plane and the adjoining black plane. The 
commensurate plane is usually selected as the origin for displacements in the mechanics description. It also represents the origin in the PTMC [23-25] and O-lattice [56, 57] theories for type I twinning and these models would also give correct transformation strains in the type I case. As discussed in detail in Sect. 4, there is also a translated commensurate dichromatic pattern, TCDC, with the black lattice shifted by a vector $\boldsymbol{p}$ relative to the white lattice in the ideal reference CDC. Finally, when there is a tiltcomponent of disconnections normal to the terrace plane there is a rotated pattern, RCDP [5]. Such patterns do not exist for TDs in type I twinning since $b_{z}$ is zero for all twin terraces, but do appear for type II twinning and, as shown subsequently, for facets on twin boundaries: only the TM treats the partitioning of rotation in such cases and the results differ from the other theories.

In an ideal Bilby bicrystal [5], all local relaxations and strains near the interface are suppressed: the two crystals have perfect lattice spacing right up to the dividing plane. Real bicrystals generally differ from ideal ones because two types of relaxation occur to reduce the interfacial free energy: first, additional rigid, relative displacement $\boldsymbol{p}$ of the two crystals away from the reference ideal bicrystal; and second, small adjustments of individual atomic positions in the vicinity of the interface, designated local relaxations. Both types of relaxation are relevant to various classes of interfacial defects, as is developed further in Section 3. Possibly, a multiplicity of distinct bicrystals with the same interface plane can exist, each with characteristic relative displacement, $\boldsymbol{p}_{\mathrm{i}}$ for the $i^{\text {th }}$ bicrystal. As mentioned in Appendix B, the untransformed matrix crystal, $\mu$, can be considered to have embedded points corresponding to $\lambda$ sites, equivalent to an embedded CDC. This representation is most useful in determining shuffle vectors, imperfect Burgers vectors, and Frank vectors for disclinations.

The real bicrystal is finally created by superposing any local nonlinear displacements that can include a relative shift $p_{z}$ of the two crystals. Local relaxations in a real bicrystal, suppressed in the ideal Bilby bicrystal, cannot be easily predicted and local displacements of individual atomic positions must be determined by TEM or by atomistic simulations. 
When considering the displacements, including shuffles, accompanying disconnection motion, a reduced portion of the CDC, defined as an exchange or e-cell, is useful. The e-cell has antecedent in the work of Bilby and Crocker [58]. A unit e-cell for the $(10 \overline{1} 2)$ twin is shown in Figure 3. Following the convention in Appendix B, the origin is selected as a $\lambda$ site on the last transformed $\lambda$ layer. The cell is defined by minimum possible translation vectors, $\mathbf{t}_{1}$ and $\mathbf{t}_{2}$ in the dividing surface and $\mathbf{t}_{3}$ inclined to it, superposed for $\mu$ and $\lambda$. It can be viewed as the minimum Voronoi cell that represents the transformation. More generally the e-cell can be larger in the $z$ direction for disconnections with larger step heights. When an ideal bicrystal like the one in Figure 3 is formed, the white crystal above the interface can be envisioned to contain fiducial embedded points corresponding to the black lattice in the CDC. Thus the $\mu$ portion of the e-cell can be considered to represent a superposition of both the CDC and the ideal Bilby bicrystal. The displacements from $\mu$ sites to $\lambda$ sites are identical in conformal mapping between the CDC and the e-cell. This concept is useful in defining shuffles. Also shown in Figure 3 is the usual $\mathbf{t}$ vector construction giving the Burgers vector. This construction is present in the e-cell when there are coincident $\lambda$ and $\mu$ sites there since the edges of the cell correspond to $\mathbf{t}$ vectors. If the two lattices are displaced at the origin, $\boldsymbol{b}$ is given by the difference in $\mathbf{t}$ vectors in the CDP.

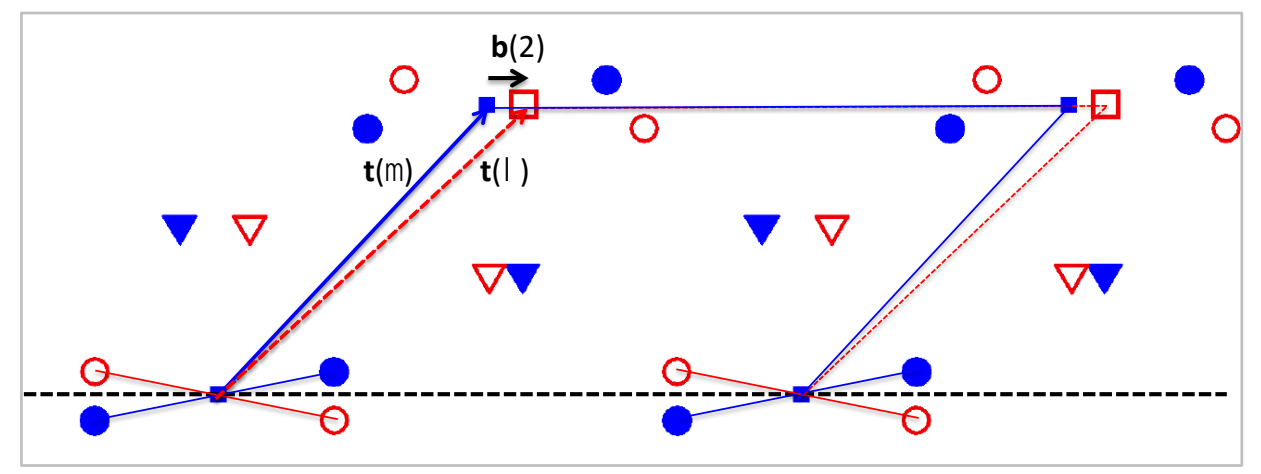

Figure 3. An e-cell corresponding to the CDC in Figure 2.

The second example is the (111) $f c c$ twin [32], a $\Sigma 3$ interface, useful in explaining type II twinning. For $f c c$ and most close-packed layer terraces, the interplanar spacing is large and first layer interactions dominate the interfacial energy. As a consequence any 
added relative shifts $p_{\mathrm{x}}$ or $p_{\mathrm{y}}$ in the $\mathrm{CDC}$, and consequently in the derived bicrystal, are negligible, and the ideal Bilby bicrystal and e-cell correspond to the real Bilby bicrystal. The CDP and the CDC are identical in this monatomic basis case, although the CDP lattice need not be fixed on atomic sites. The CDC and e-cell for a unit TD are given in Figures 4(a) and 4(c). Components of multiple height TDs, Sect. 3.6, can have out-of plane Burgers vectors as shown in Figures 4(b) and 4(d). We emphasize that these are reference crystals which suppress the local strains near the TDs in a real bicrystal. For less close-packed terraces, the interplanar spacing is relatively small and the minimum energy configuration is typically displaced from the coincident configuration. The real bicrystal is finally created by superposing any local nonlinear displacements that can include a relative shift $p_{z}$ of the two crystals.
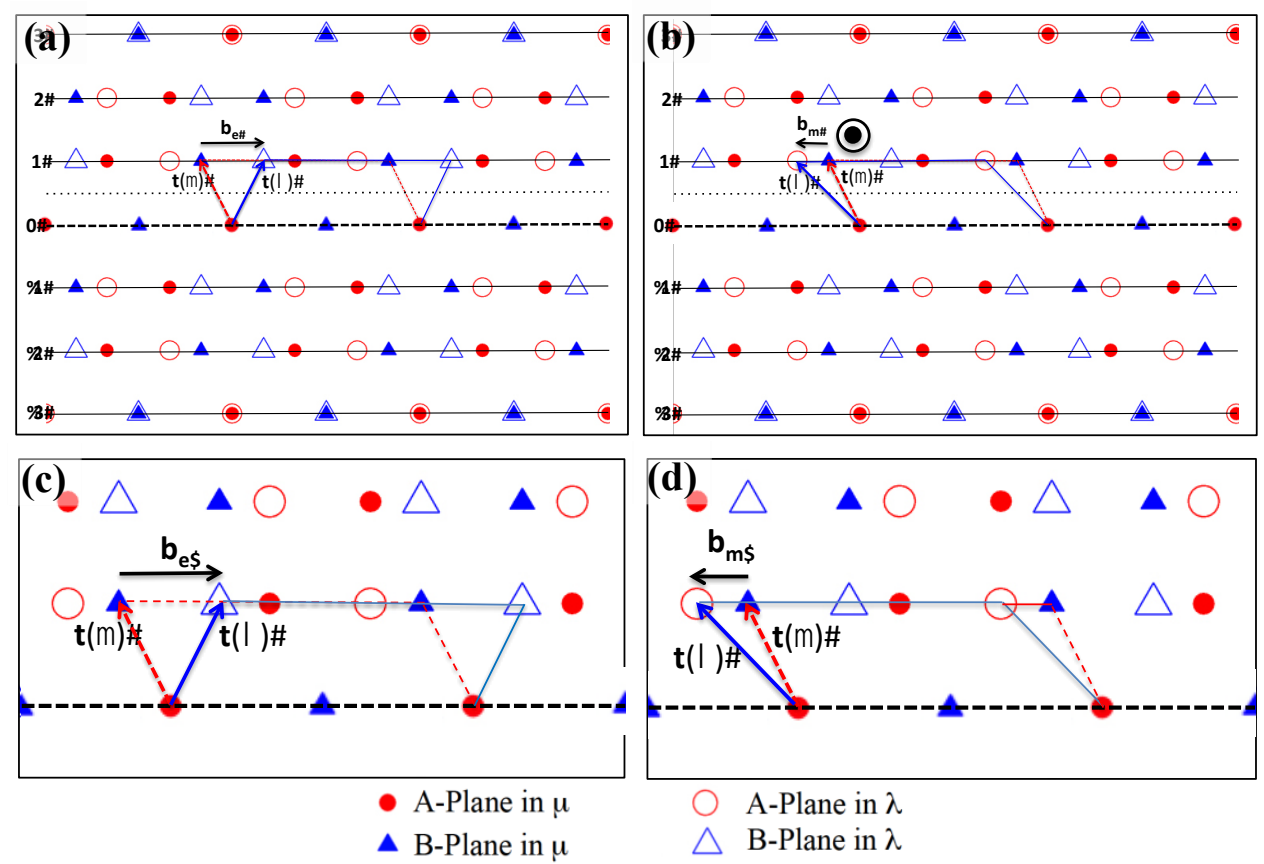

Figure 4. (a) CDC for $f c c$ (111) twins viewed along [1ํㅣㄹ. In-plane Burgers vector $\boldsymbol{b}_{\mathrm{e}}$ is shown, (b) CDC for mixed, out-of plane dislocation $\boldsymbol{b}_{\mathrm{m}}$. Respective e-cells shown in (c) and (d).

The third interface is the $f c c$ (301) twin interface for a $\Sigma=5$ CSL [56]. This is of interest in that, although the basis is monotonic, the unit disconnection can be multiple planes in height. A square lattice containing all atoms in Figure 5 comprises a DSC 
lattice, again showing equivalence with the O-lattice [56, 57] for this special case. As indicated, with the restriction to plane strain displacements (another possibility is treated later), there are two possible disconnections with $\boldsymbol{b}(2), h(2)$ and $\boldsymbol{b}(4), h(4)$ and two above with opposite displacements, related by the 2 -fold rotation axis $2 \mathrm{x}$. The minimum energy case is evidently $\boldsymbol{b}(2)$ and $h(2)$. The e-cell for $\boldsymbol{b}(2), h(2)$, is given in Figure 5 . The notation for step heights is presented in Appendix C. In other twins, larger step height disconnections can be favored by having a lower energy, small $\boldsymbol{b}$. An $h(4)$ defect of this type for a $\Sigma 11 \mathrm{fcc}$ twin is analysed in [59], and was observed in TEM [60].

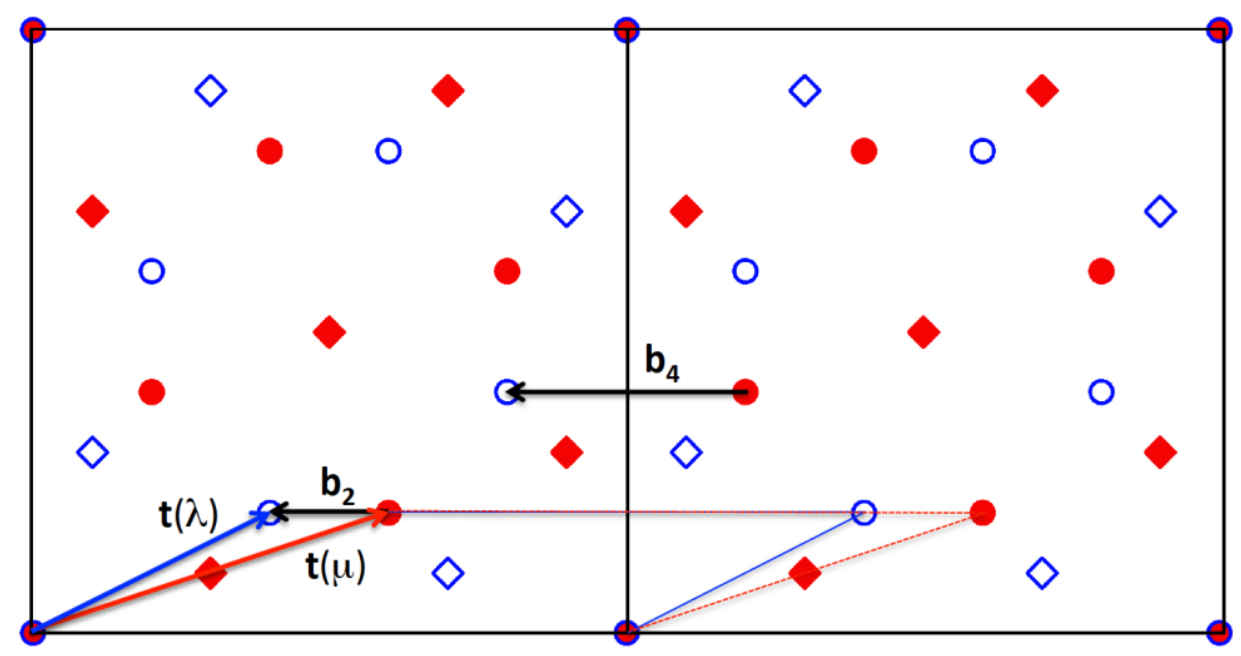

Figure 5. CDC for (301) twin in $f c c$ metals. An e-cell is outlined at bottom. Here y // [1 $\overline{3} 0]$ and $\mathrm{z} / /[301]$.

\subsection{The Gibbs interface}

There is a thermodynamic excess volume for all interfaces, although it may be negligibly small in some cases. In order to measure excess volume, one must define a Gibbs reference interface [61]. A way of doing this for a twin terrace, that works as well for free surfaces, is to define the Gibbs interface as one corresponding to the twin interface, shifted from the commensurate plane by $\pm d / 2$ in the $z$ direction, where $d$ is the appropriate spacing of the planes. For a phase boundary or for interfaces such as the prismatic-basal $(\mathrm{P} / \mathrm{B})$ boundary [37] with different $\mathrm{d}$ spacings normal to the terrace another procedure is needed. First, the Poisson strains are neglected since they are cancelled by misfit strains in an equilibrium boundary. Second, as illustrated in Figure 6, 
the spacing at the interface is $1 / 2\left(d_{\mu}+d_{\lambda}\right)$ and the interface is at the midpoint of this spacing.

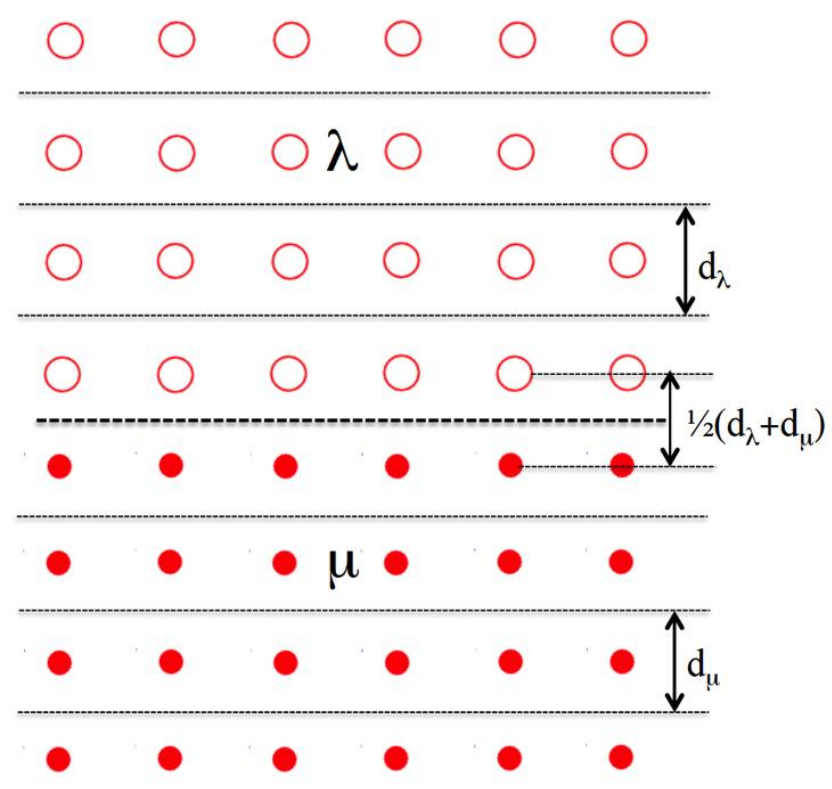

Figure 6. Gibbs reference interface.

Since $p_{\mathrm{z}}$ is normal to the terrace plane, and is the shift accompanying the transformation of a unit plane from white, $\lambda$, to black, $\mu$, it represents an excess volume $\delta^{e}$ per unit area of the terrace plane, relative to the Gibbs reference interface.

$$
\delta^{e}=p_{z}
$$

Two examples of excess volume are given in Figure 7 for $\mathrm{Cu}$ and $\mathrm{Nb}$, respectively. Density function theory (DFT) calculations, at $0 \mathrm{~K}$, of the twin boundary and intrinsic stacking fault free energy, $g_{\mathrm{t}}$ and $g_{\mathrm{f}}$, respectively, were performed as a function of $p_{\mathrm{z}}$. For the $f c c \mathrm{Cu}$ twin, $g_{\mathrm{t}}=19.2 \mathrm{~mJ} / \mathrm{m}^{2}$ at a value of $p_{\mathrm{z}}=\delta^{\mathrm{e}}=0.02 \mathrm{~nm}$. For the fault, $g_{\mathrm{f}}=40 \mathrm{~mJ} / \mathrm{m}^{2}$ at a value of of $p_{\mathrm{z}}=\delta^{\mathrm{e}}=0.03 \mathrm{~nm}$. The free energies are in good agreement with typical experimental results, summarized in Appendix 2 in [62]. For bcc $\mathrm{Nb}, g_{\mathrm{t}}=277 \mathrm{~mJ} / \mathrm{m}^{2}$ at a value of $p_{\mathrm{z}}=\delta^{\mathrm{e}}=0.04 \mathrm{~nm}$. Measurements from TEM give $\delta_{\mathrm{e}}=$ $0.02 \mathrm{~nm}$ for a twin boundary in Al [63]. Thus all examples have a positive excess volume, usually significant enough to be detectable with advanced electron microscopy techniques. 
These relaxations, $p_{z}=\delta^{\mathrm{e}}$ are localized at the interface [64]. They produce no long-range distortions. Therefore, they appear in real bicrystals, the final stage in the TM analysis, but do not appear at any intermediate stage, represented by the patterns, the complexes, the ideal bicrystal or the e-cell.

For other interfaces, such as the $\mathrm{P} / \mathrm{B}$ interface in $h c p$, the origin for the e-cell generally differs from that in Figure 6. Thus, while $\delta_{\mathrm{e}}$ contributes to $p_{\mathrm{z}}$, there are other contributions to the latter as well, and the former is best considered as a separate thermodynamic property.

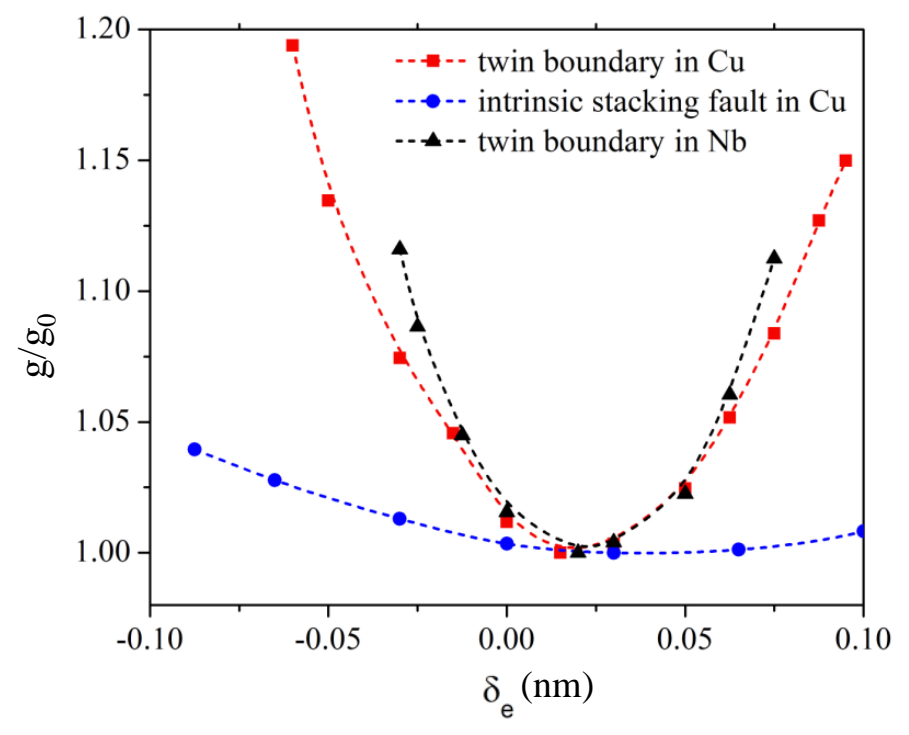

Figure 7. Variation of the normalized interface energy with the shift vector $\delta_{\mathrm{e}}$, the normalization factor $\mathrm{g}_{0}$ is $19.2 \mathrm{~mJ} / \mathrm{m}^{2}, 40 \mathrm{~mJ} / \mathrm{m}^{2}$, and $277 \mathrm{~mJ} / \mathrm{m}^{2}$ for a twin boundary and an intrinsic stacking fault in $\mathrm{Cu}$ and a twin boundary in $\mathrm{Nb}$, respectively.

\section{Interfacial Line-Defects}

\subsection{Defect character}

Our objective in this section is to illustrate the topological character of defects that arise in interfaces based on terrace structures. We concentrate on defects relevant to interface motion, and these exhibit dislocation, disclination, or step character, or some combination thereof. Defects can either separate terrace segments with the same normal, 
$\boldsymbol{n}$, or facets with different normals. We refer to the former case as terrace defects, and the latter as junction defects. Terrace defects are discussed first and then junction defects.

\subsection{Terrace dislocations and disconnections}

There are three categories of terrace dislocations/disconnections: perfect, domain and imperfect defects, as defined separately below.

\subsubsection{Perfect defects}

The formation of a perfect defect is illustrated schematically in Appendix A, Figure A-1b: the white and black crystals are depicted with appropriately prepared surfaces, each one exhibiting a step, where the step risers correspond to white and black translation vectors $\boldsymbol{t}(\lambda)$ and $\boldsymbol{t}(\mu)$. As described in Appendix A, the Burgers vector of the disconnection is

$$
\boldsymbol{b}=\boldsymbol{t}(\lambda)-\boldsymbol{t}(\mu)
$$

i.e., the difference between $\boldsymbol{t}$ vectors expressed in the white crystal coordinate frame. This vector is independent of any rigid shift $\boldsymbol{p}$ of $\mu$ relative to $\lambda$ because the terraces bounding perfect defects are identical, i.e., they are both characterised by the same rigid displacement, $\boldsymbol{p}$. The step height $h$ of the disconnection is relevant to the orientation of the interface and the calculation of the long-range diffusive flux accompanying defect motion [49]. For a given terrace there may be a multiplicity of admissible disconnections, and, for ease of reference, we represent a particular defect using the abbreviated notation $\boldsymbol{b}(m, q), h(m, q)$, where $m$ and $q$ identify the integer number of atomic planes in the notional surface steps, when the step heights differ for $\mu$ and $\lambda$ and $\boldsymbol{b}(m), h(m)$ when the heights are the same as for TDs. Thus the heights are given by

$$
\begin{aligned}
& h=h(m, q), \quad h(\lambda) \neq h(\mu) \\
& h=h(m), \quad h(\lambda)=h(\mu) \\
& m=\boldsymbol{n} \cdot \boldsymbol{t}(\lambda)_{\boldsymbol{i}} / d(\lambda), q=\boldsymbol{n} \cdot \boldsymbol{t}(\mu)_{j} / d(\mu)
\end{aligned}
$$


Translation vectors, $\boldsymbol{t}(\lambda)$ and $\boldsymbol{t}(\mu)$, define the $\lambda$ and $\mu$ lattices respectively, and their union. Here, $d_{\lambda}$ and $d_{\mu}$ are the spacings of atomic planes parallel to the terrace in the white and black crystals respectively. The length of $h$ is selected as the smaller of $h(\lambda)$ or $h(\mu)$. The infinity of translation vectors, $\boldsymbol{t}(\lambda)_{i}$ and $\boldsymbol{t}(\mu)_{j}$, define the $\lambda$ and $\mu$ lattices respectively, and their union defines the CDP: an examples of a CDP for two hexagonal rotated by $87.3^{\circ}$ about a common [ $\left.\overline{1} 2 \overline{1} 0\right]$ direction, creating the CDP for (1012) twinning in $\mathrm{Mg}$ with $c / a=1.623$ is shown in Figure 2(a). The utility of such CDPs is that the parameters $\boldsymbol{b}(m)$ and $h(m)$ for admissible perfect defects are depicted graphically for the particular case: they can be "read" from the CDP. Corresponding simulated terraces separated by a twinning disconnection (TD) are shown in Figure 8 and a TEM image of such a TD in Ti is shown in Figure 1(d). In this case, the terrace is naturally coherent and any local distortions are so small that the terrace is essentially equivalent to that in an ideal reference bicrystal. In this case, the (1012) terrace is naturally commensurate.

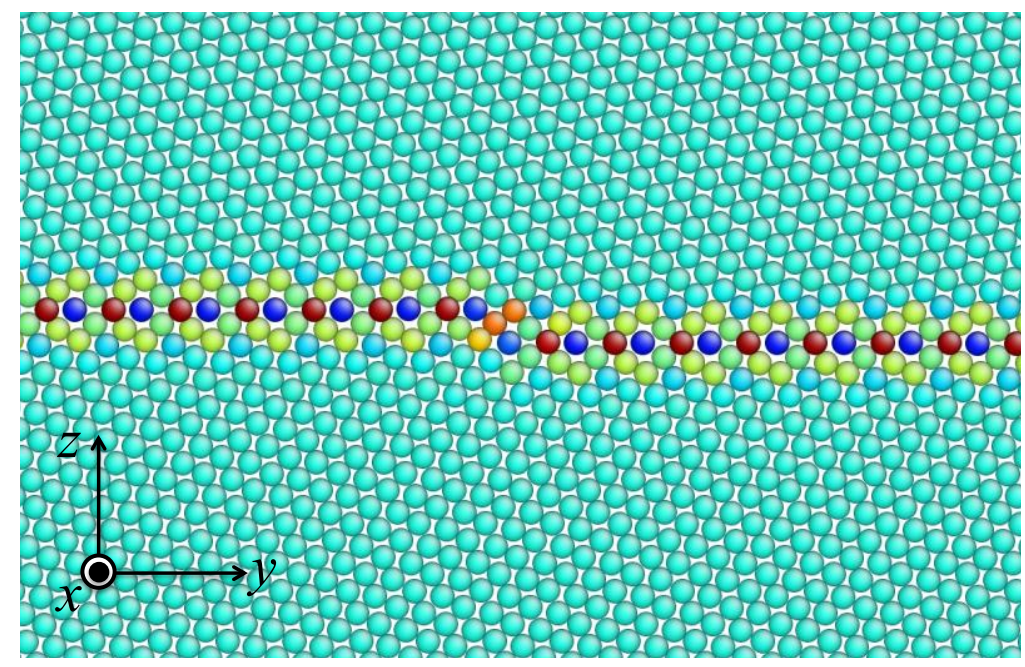

Figure 8. Simulated twin plane/terrace for a (101) 2 twin in Mg, present work. An $h(2)$ disconnection is present. Atoms are coloured according to their excess energy. Here $\mathrm{x}$ is along [1릴, $\mathrm{y}$ is along the twinning shear, and $\mathrm{z}$ is along the normal of the twin plane (101̄2).

Subcategories of the ubiquitous, perfect interfacial defects are defined in a manner reflecting their functions, as follows: 
(a) Twinning disconnections (TDs) are unit disconnections responsible for twinning. They move along the twin terrace conservatively: the disconnection $h(2)$, indicated in Figures 2 and 8, is an example. For TDs, conservative motion follows since the atomic densities are the same for a given $m=q$, fulfilling the conservation condition [37].

(b) Crystal dislocations originate in one of the crystals and reach the interface by motion on a glide plane inclined to the interface. For brevity, we designate these as slant dislocations. Here, either $m$ or $q$ equals zero, and $\boldsymbol{b}$ correspondingly equals $\boldsymbol{t}(\lambda)$ or $\boldsymbol{t}(\mu)$ when referred to the crystal from which the defect emanated. Dislocations could move by climb to join the interface, but this process is usually negligible for the deformation twinning case and is not considered here.

(c) Misfit dislocations are crystal dislocations where there is a component of $\boldsymbol{b}$ is parallel to the terrace. (It follows that both $m$ and $q$ equal zero). The case of $\boldsymbol{b}$ parallel to the interface is the most efficient arrangement for accommodation of misfit. For the large bicrystal, homogeneous, isotropic elastic case, the misfit strains are equally partitioned $[17,65,66]$. With anisotropic or inhomogeneous elasticity, or with unequal layer thicknesses, partitioning occurs, but the strains are no longer equal in the two crystals [65-67].

(d) Tilt dislocations arise in symmetrical tilt boundaries for example, and can be formed by the reaction of symmetrical pairs of black and white slant dislocations. In that case, the tilt boundary can be regarded as having equal numbers of slant dislocations from each crystal or as grain boundary dislocations (gbds) with their Burgers vectors $\boldsymbol{b}_{g}=1 / 2[\boldsymbol{t}(\lambda)+\boldsymbol{t}(\mu)]$ perpendicular to the interface. The magnitudes are equal to $2 b \cos (\varphi / 2)$, where $b$ is the magnitude of the crystal dislocation Burgers vector, and $\varphi$ is the angle of misorientation [18]. Since $m=-q$ for such defects, their overlap step height is zero. The rotation associated with the tilt wall must be equally partitioned to the two crystals in the homogeneous, isotropic case [53,67].

(e) Pure steps have no dislocation character. Pure steps can only arise when a CDP exhibits coincident vectors, $\boldsymbol{t}(\lambda)_{i}=\boldsymbol{t}(\mu)_{j}$, inclined to the terrace. This is true for a 
disconnection with $h(3)$ for a (111) $f c c$ twin but not for unit disconnections (those with minimum possible step height $h(1)$. Pure steps are more common in $f c c$ crystals where CSLs are defined as coincident lattices. More generally, for cases where $h$ is very large, Sect. 3.5, misfit dislocations can arise on the step riser so that, overall, $\boldsymbol{b}$ can be very small, even though a pure step may be impossible to achieve.

\subsubsection{Domain defects}

Domain defects do not arise as frequently as perfect ones, but are important in particular instances. Their topological origin is described formally in [1]. Domain defects separate energetically and structurally degenerate terraces, interrelated by the orthogonal part of a symmetry operation, $\boldsymbol{W}$. This symmetry is common to the two lattices in the $\mathrm{CDP}$, but is not present when all the atomic sites are considered, i.e. in the corresponding CDC.

Domain defects, where the terraces adjoining the defect are energetically degenerate but rotated with respect to one another, are relatively rare in twinning. Experimental observations of domain defects with dislocation character are reported in [1]. They are most commonly formed as a consequence of local reactions arising at an intersection of an extrinsic dislocation with a terrace. An example of such domain dislocations and disconnections has been studied in detail in $\{\overline{2} 11\}$ twin boundaries in $\mathrm{Al}$ [69-71].

Domain defects without dislocation character can also arise in the final step of converting a Bilby ideal bicrystal to a real bicrystal. Here, local relaxation at the interface, rather than a rigid-body displacement, breaks either coincident or antisymmetry. Such interfacial domain lines separate degenerate terrace segments with structures interrelated by the broken symmetry. This is analogous to domain lines that occur by virtue of symmetry breaking reconstructions on crystal surfaces [1, 2]. A possible observation of an interfacial domain line has been reported in [69].

\subsubsection{Imperfect defects}


Imperfect defects separate terrace segments with the same normal, $\boldsymbol{n}$, but with distinct structures, and are characterised by Eq. (A3). Partial dislocations bounding a stacking fault in $f c c$ are a simple example. However, if the distinct structures have sufficiently different free energies, spontaneous formation of a fault bounded by imperfect defects is most unlikely. When they do form it is probable that they arise from the dissociation of an extrinsic dislocation at an interface, where the gain in elastic energy offsets the fault energy. An example of an imperfect dislocation in Ge has been studied experimentally by Bacmann et al. [72]; Here, there was no step character. One with step character has been observed in $\mathrm{GaN}$ [73].

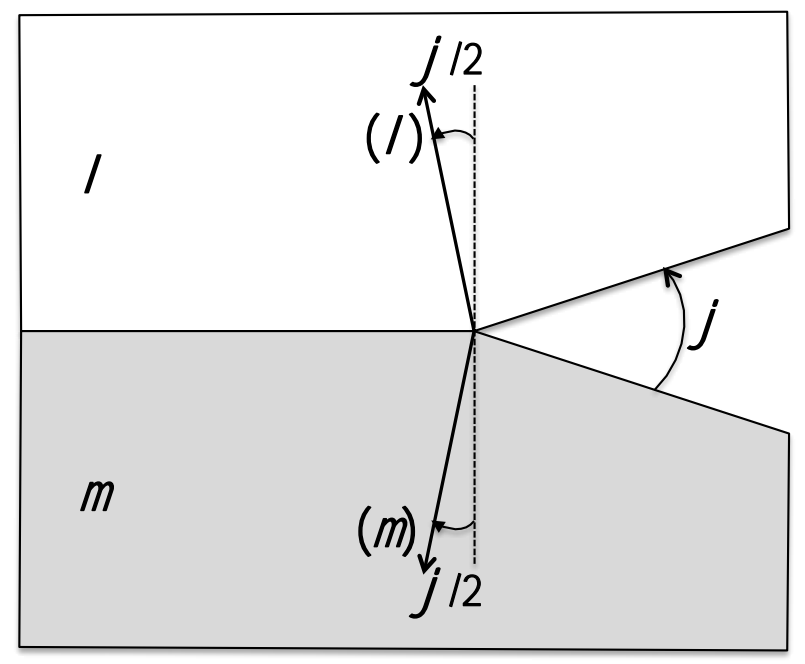

Figure 9. Volterra representation of a disclination. This can be mapped into a CDC, where the displacements and rotations associated with closing the gap to create the disclination can be mapped.

\subsection{Terrace disclinations}

We introduce this discussion by briefly reviewing the properties of and conventions for disclnations in single crystals. Perfect disclinations in the bulk of a single crystal are characterised by a symmetry operation which is a proper rotation, $\mathfrak{w}_{\boldsymbol{i}}$. However, since the smallest admissible angle is $\varphi=60^{\circ}$, such defects are improbable in large crystals of stiff materials [12]. They can form in animate and inanimate organic compounds [13] and in magnetic or elecrostatic domain lattices [74], all of which have soft elastic constants. Imperfect disclinations, with smaller rotation angles, can form in 
harder materials, and terminate a "fault plane", such as a discontinuous tilt or twist wall, or a twin boundary: all disclinations treated here are imperfect. Thus, an imperfect disclination is characterised by a non-symmetry operation, yielding a rotation matrix, and rotation by an angle $\varphi$ about a specified axis. Hence, one can envision the rotation in terms of the matrix $R$ or the rotation angle $\varphi$. The representation in a CDP is shown in Figure 9. The rotation is part of the elastic distortion associated with the disclination, and, just as for a tilt wall, must be partitioned to the two crystals as shown in Figure 9.

Based on the Seitz convention [75] a formal analysis of $\boldsymbol{R}$ in the topological theory is given in [76]. Disclinations are not topologically distinct from dislocations in the sense that they can be modelled as a terminating wall of dislocations [62]: for consistency with the RH/FS convention for defining the sense of Burgers vectors, the disclination line direction, $\boldsymbol{\xi}$, is toward the viewer in Figure 9, i.e. $\boldsymbol{R}$ is a right-handed rotation relative to $\xi$.

The stress field of perfect and imperfect disclinations can be described conveniently in terms of a topologically equivalent dislocation array $[62,77]$. Thus, for an isolated imperfect disclination passing through the origin, lying along $x$, and where the fault plane is the $x z$ plane, the shear stress varies as

$$
\tau_{y z}=\frac{\mu_{M} b y z}{2 \pi(1-v) D\left(y^{2}+z^{2}\right)}
$$

and other stress components vary similarly [77]. Here, $\mu_{M}$ is the shear modulus (subscript $\mathrm{M}$ to distinguish this parameter from the bicrystal designation $\mu$ ), and the component dislocations have Burgers vectors with magnitude, $b$, and spacing $D$ : these dislocations either can be discrete, as in a low-angle boundary, or $b / D$ may represent the Burgers vector density of an array with infinitesimal Burgers vectors [28]. Equation (5) shows that the long-range stress field is equivalent to that of a super-dislocation with "Burgers vector" $y b / D=2 y \sin (\varphi / 2)$. The super-dislocation therefore has a stress field divergent in y. For most cases of interest, the disclinations are best envisioned in the CDP, which is the procedure used here. 
Of more interest for interface defects is the disclination dipole. The long-range field of a disclination dipole is equivalent to that of a dislocation $[78,79]$. This can be proved by the topological theory, but also follows directly from the discontinuous tilt wall analog. For a terminated discontinuous tilt wall of length $d$ in the y direction, the dipole stress field is given by [62],

$$
\tau_{y z}=\frac{\mu_{M} b}{2 \pi(1-v) D}\left[\frac{y^{2}}{\left(y^{2}+z^{2}\right)}-\frac{y^{2}}{\left(y^{2}+(z+d)^{2}\right)}+\frac{1}{2} \ln \frac{\left(y^{2}+(z+d)^{2}\right)}{\left(y^{2}+z^{2}\right)}\right] .
$$

In contrast to the single disclination, the long-range field scales logarithmically with $d$. As seen in Eq. (6) the ratio $b / D$ scales with the stress and strain field of the dipole. If one divides $\mathrm{b}$ into smaller portions, $\mathrm{D}$ is divided by the same factor. Hence the long-range stress, Eq. (6), is independent of the division. This proves that the discontinuous tilt wall dipole and the disclination (with infinitesimal dislocations) have the same long-range field. Equation (6) is valid for both unit dislocations/disconnections and larger facets. For unit defects, the short-range distortion field differs from that of a dislocation [80].
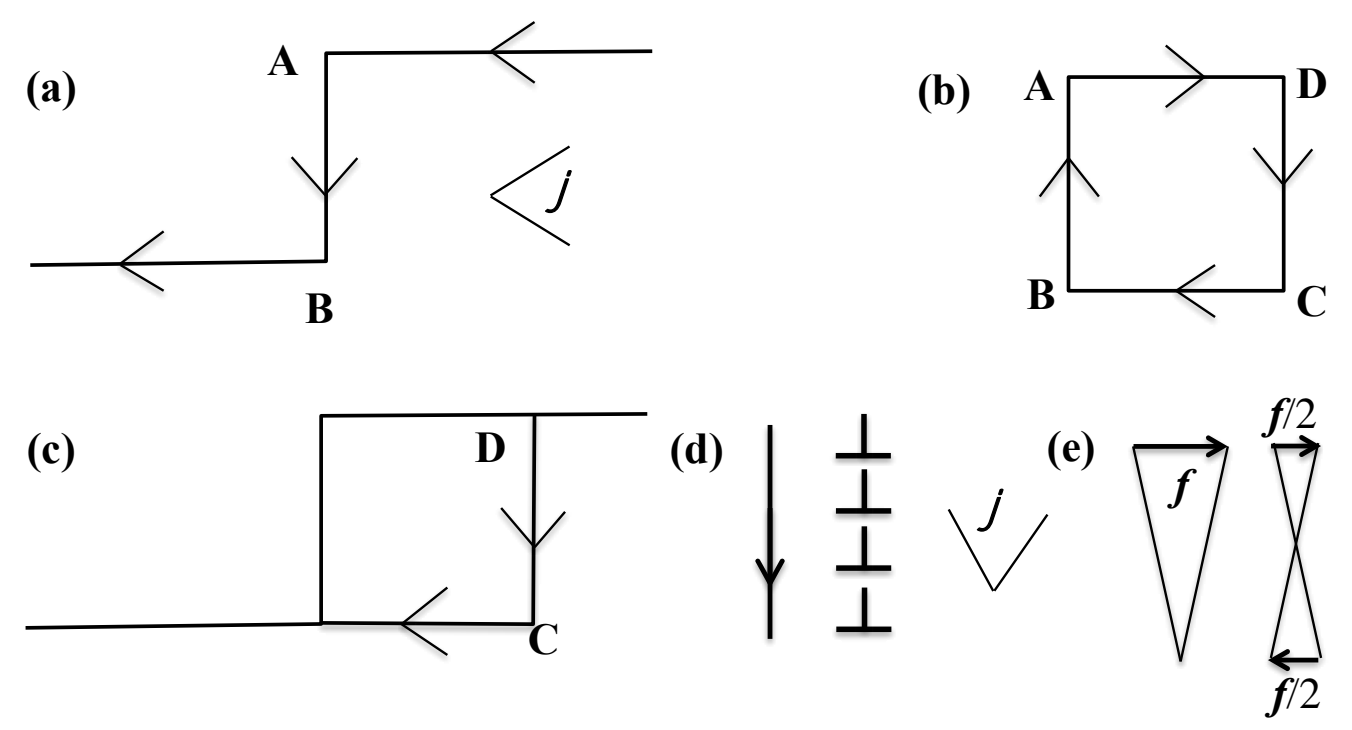

Figure 10. (a) Step represented as a disclination dipole with characteristic angle $\varphi$. (b) Disclination quadrupole. (c) Superposition of (a) and (b) producing the advance of the step.

\subsection{Junction defects}

\subsubsection{Junction line disclinations}


The long-range field is equivalent to that produced by a dislocation content with a total Burgers vector equal to $f$, known as the Frank vector [81], represented in Figure 10. We see that

$$
f / 2 d=\sin (\varphi / 2)=b / 2 D
$$

connecting the two approaches. Formally, the vectors $\boldsymbol{f}$ and $\boldsymbol{d}$ and the rotation $\boldsymbol{R}$ are defined by mapping into the appropriate (rotated) RCDP or RCDC $[5,76]$. That is, $\boldsymbol{f}$ is a grain boundary dislocation Burgers vector, normal to the interface, and in the RCDC is the vector sum of two $t$ vectors, Figure 10, again demonstrating the importance of partitioning of the rotation. For unit step heights the Frank vector $\boldsymbol{f}$ is better regarded as a discrete dislocation (the sum of the infinitesimal dislocations in the step), since any smearing is in a nonlinear core regime. For most cases of interest, the disclinations are best envisioned in the CDP, which is the procedure used here. Any corrections associated with basis vectors can be superposed as needed.

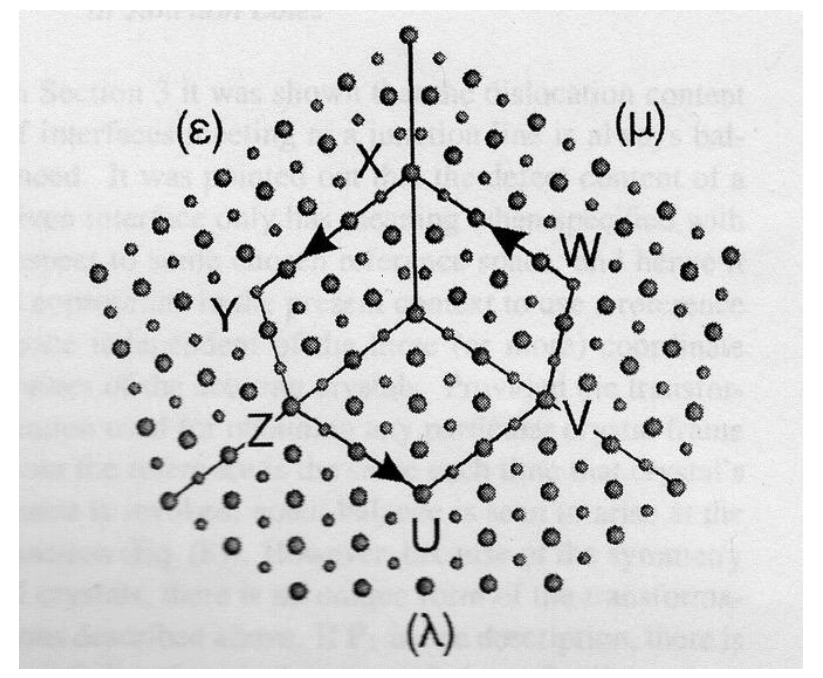

Figure 11. Three-fold junction line [82].

We are concerned primarily with disclinations delineating the junction of two degenerate or distinct terraces. However, more than two interfaces might meet at a junction line: an important example is a triple junction line in a polycrystal [82], as depicted in Figure 11. We designate the three grains, white, $(\lambda)$, black, $(\mu)$, and red $(\epsilon)$, and the net rotational matrix as $\boldsymbol{R}=\boldsymbol{R}_{\epsilon \lambda} \boldsymbol{R}_{\mu \epsilon} \boldsymbol{R}_{\lambda \mu}$. The junction line is free of long-range 
stress if $\mathbf{R}=\boldsymbol{I}$, but otherwise exhibits defect character [1]. Equivalently, the condition for a perfect, stress free junction can be expressed as the sum

$$
\phi_{\epsilon \lambda}+\phi_{\mu \epsilon}+\phi_{\lambda \mu}=0
$$

This rule for an equilibrium junction free of long-range stresses as a continuity theorem is analogous to that for dislocation Burgers vectors meeting at a node [62]. A well-known example of a multi-junction line is that exhibited at the junction of five-fold twinning in small $f c c$ composites, Figure 12 [83], which exhibits a field equivalent to a $7.35^{\circ}$ disclination. TEM observations of junction disclinations in $\mathrm{YBa}_{2} \mathrm{Cu}_{3} \mathrm{O}_{7}, \mathrm{TiH}: \alpha-\mathrm{Ti}$ and NiBe: $\alpha-N i$ have been analysed by Dimitrakopulos et al. [84]. Figure 13 is an image of a disclination dipole at junctions of a tetragonal $\mathrm{TiH}$ precipitate in a matrix of hcp Ti [85]. Here, the imperfect disclinations accommodate rotations of $\pm 30^{\circ}$ between degenerate interfaces, and, because of its small size, virtually all the rotational distortion is partitioned to the precipitate. Nevertheless, the distortions the distortions are so severe that the dipole is unstable and was observed in the TEM to transform into a tilt boundary separating the two arms of the precipitate [85].

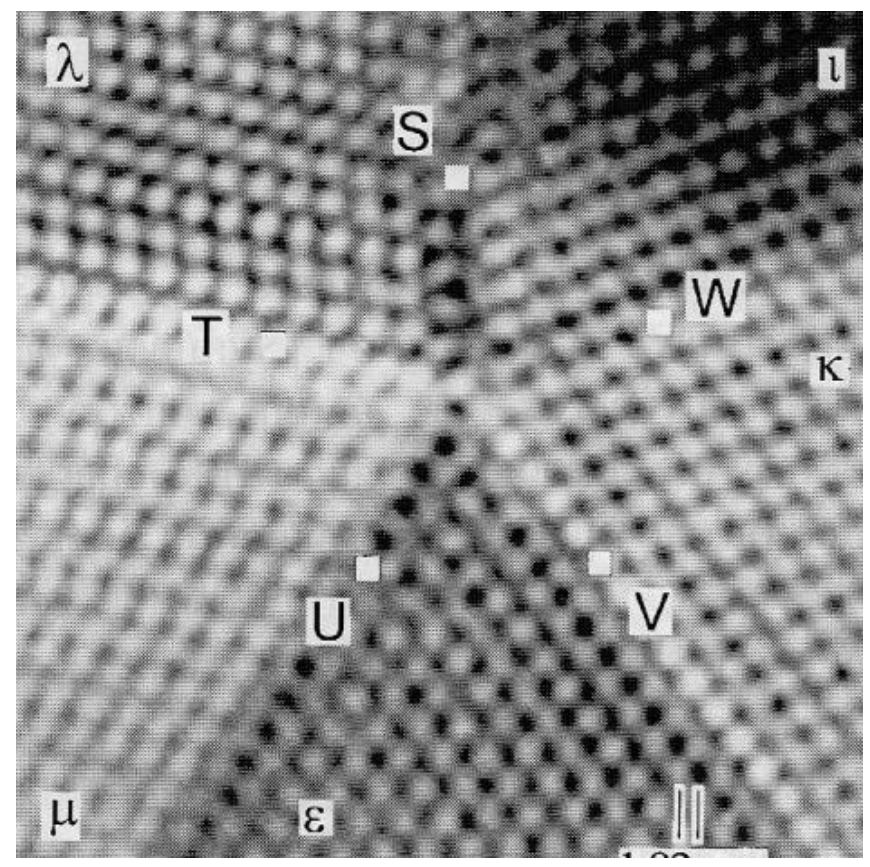

Figure 12. TEM image of 5-fold twinning in Si [83], producing a five-fold junction line. 


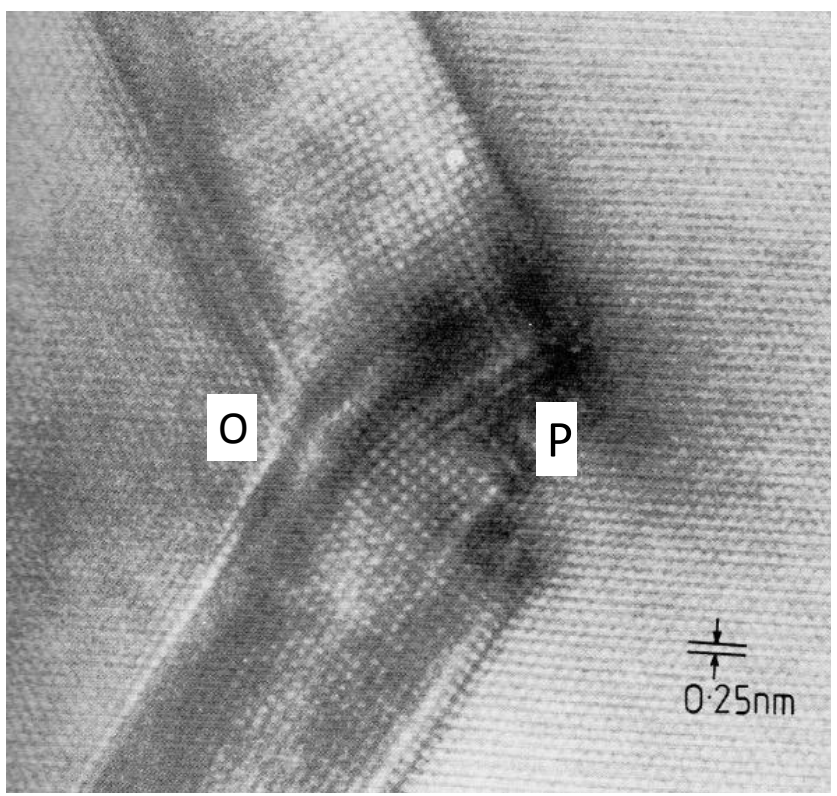

Figure 13. Disclination dipole at junctions of a tetragonal TiH precipitate in a matrix of $h c p$ Ti [85].

Finally, we consider pure steps or the step portions of dislocations, which can also be regarded as perfect dislocation multipoles. This has the important consequence of physically representing the local rotation (at the e-cell level) produced by step motion. Figure 10(a) shows a step interpreted as an array of disclinations. Here, the misorientation across the step-riser, $\varphi$, is the same as that across the terraces, $\varphi$, so no discontinuities arise at the lower or upper junctions and there is no long-range distortion field: Eq. (8) is satisfied. However, each terrace, if isolated, would comprise a disclination and the step riser a disclination dipole. Both coherent interfaces are characterized by the same rotation. This concept is useful in modeling the motion of the step, where one can imagine that disclination dipoles delineate the two junction lines. In Figure 10(a) the junction lines are at A and B. In Figure 10(b) the lines are at A, B, C and D and comprise a disclination quadrupole. Superposition of Figures 10(a) and 10(b) advances the step to Figure 10(c), the new position CD. Hence, the disconnection can be considered to contain a disclination quadrupole that produces a pure rotational reorientation as the disconnection moves [86]. With regard to the presence of net disclination character, this association of step motion and rotation is true whether or not the step interface is degenerate with the terraces. In either case, junction line dislocations 
are present as discussed next. In the discrete dislocation analog, the absence of longrange stresses is known for so-called L-junctions [87].

Thus, the separation of the step portion of a disconnection, and the dislocation portion can have significance other than for the analysis of the motion in e-cells. The two separated portions have differing physical significance, step motion producing pure rotation and dislocation motion producing distortion (shear plus rotation).

If the step riser (facet) is characterized by a different rotation angle $\varphi+\varphi^{0}$ relative to the terrace $\varphi^{0}$ then there would be a net stress field. The field is that of a superposed disclination dipole, Figure 10(d), with a stress field associated with the difference in angles, $\varphi$. The tilt wall equivalent is also shown. Either representation produces a local stress field given by Eq. (6) [62]. Significantly, the stresses of Eq. (6) diverge at both ends of the facet (at $y=0$, and either $z=0$ or $z=-d$ in coordinates fixed on the facet). As a consequence the rotation must be partitioned as indicated in Figure 10(e): to our knowledge this necessity has not been previously made explicit. The partitioned disclination is effectively two disclinations with the same $\varphi$ meeting at a coincident midpoint that is stress free by condition (8). Additionally, the Frank vector is partitioned into two stress sources with vectors $\pm f / 2$ at the singularities. This partitioning is not considered in the extensive gauge theory of disclinations.

\subsubsection{Junction line dislocations}

These dislocations delineate junctions separating either distinct or degenerate terraces. Consider first the junction between two terraces with distinct structures, designated $\alpha$ and $\beta$ : their normals are $\boldsymbol{n}_{\alpha}$ and $\boldsymbol{n}_{\beta}$, and the relative displacements are $\boldsymbol{p}_{\alpha}$ and $\boldsymbol{p}_{\beta}$ respectively. If this difference is accommodated, a dislocation with Burgers vector

$$
\boldsymbol{b}_{\alpha \beta}=\boldsymbol{p}_{\alpha}-\boldsymbol{p}_{\beta}
$$

must be present. In addition there would be a shuffle with vector

$$
\boldsymbol{s}_{\alpha \beta}=\left(\mathbf{p}_{\alpha}+\mathbf{p}_{\beta}\right) / 2
$$


A similar situation arises for two degenerate terraces, except that the normals and relative displacements are interrelated by some coincident or anti-symmetry element.

Junction dislocations have been observed. A well-known example is the stair-rod dislocation at the junction of intrinsic stacking-faults in $f c c$ crystals [62]. More familiarly the stair-rod vector, $\boldsymbol{b}_{a b}$, is denoted $\boldsymbol{\delta} \boldsymbol{\gamma}$ using the Thompson tetrahedron [62]. Junction dislocations separating degenerate pairs of $\{211\}$ terraces of faceted annealing twin boundaries have been studied experimentally using TEM in Al [68] and Si [88, 89]. Their Burgers vectors were found to be consistent with the treatment presented here, and the magnitudes $\left|\boldsymbol{b}_{\alpha \beta}\right|$ were determined to be $0.39 a$ and $0.49 a$ for Al and Si respectively, where $a$ is the relevant lattice parameter. Dislocations demarcating $\{211\} /\{171\}$ terrace junctions, i.e. junctions of distinct terraces, were found to have $\left|\boldsymbol{b}_{\alpha \beta}\right|=0.18 a$ in $\mathrm{Al}$ [68].

\subsection{Multiple height TDs}

The TDs discussed to this point are mainly unit TDs of a given type, defined by the shortest perfect disconnection translation vector pair, $\boldsymbol{t}_{\lambda}$ and $\boldsymbol{t}_{\mu}$; unit indicating perfect and with a specified minimum length Burgers vector. Multiple defects with large step heights can form by the combination of the unit TDs. Such defects are described in a general way in [51, 90-94]. Indeed, the tip of a deformation twin or facet can be relaxed by emissary dislocations [95-97], resulting in the equivalent of a multistep. Moreover, as for $f c c$ (111) twins, the same twin can be formed by different twinning dislocations. In the Thompson notation these are $\mathbf{\delta A}, \boldsymbol{\delta} \mathbf{B}$, and $\delta \mathbf{C}$ on the (d) plane. The total Burgers vector is now

$$
\boldsymbol{b}_{\mathrm{Tot}}=\sum_{j} n_{j} \boldsymbol{b}_{j}
$$

Here $j$ is the number of different disconnection vectors (three for $f c c$ ) and $\boldsymbol{b}_{\mathrm{j}}$ is the $j$ th Burgers vector. The total step height $H$ is given by a similar sum. In terms of the Bilby representation, where the dislocations are smeared into a continuous infinitesimal array of strength $\boldsymbol{B}$ per unit step height, this total vector also $\boldsymbol{B} H$. A simple example of this is the partial bounding an extrinsic stacking fault in $f c c$ (see Fig. 10-8 in [62]). The unit dislocations might be $\boldsymbol{b}(1)=\mathbf{B} \boldsymbol{\delta}$ and $\boldsymbol{b}(2)=\mathbf{C} \boldsymbol{\delta}$, but the total long range field of the combined defect is that of a disconnection with $h(1)$ and $\boldsymbol{b}(1)=\boldsymbol{\delta} \mathbf{A}$. As shown in Figure 
14(a), the displacement at the (1) level is that of a Burgers vector $\delta \mathbf{A} / \mathbf{2}$ and a shuffle $\mathbf{C B} / 2$, while that at the (0) level is that of a Burgers vector $\delta \mathbf{A} / \mathbf{2}$ and a shuffle $\mathbf{B C} / 2$. The same shear-shuffle situation can arise at twin boundaries. Two coplanar vectors, as in Figure 3, suffice for Eq. 11 to give any direction for $\eta_{1}$, evidently making the mechanistic interpretation difficult and perhaps having a role in distinguishing type I and type II

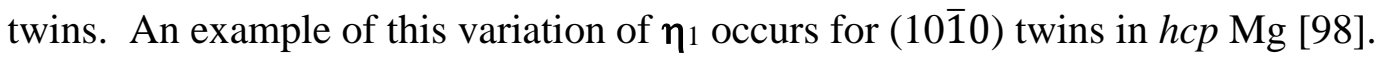

A pure step with $h(3)$ is similarly equivalent to the superposition of three dislocations with Burgers vectors $\delta \mathbf{A}, \delta \mathbf{B}$, and $\delta \mathbf{C}$, summing to give zero Burgers vector [99] and three glide-shuffle vectors $\boldsymbol{s}(1)=\delta \mathbf{A}, \boldsymbol{s}(2)=\delta \mathbf{B}$, and $\boldsymbol{s}(3)=\delta \mathbf{C}$, Figure 14(b). Both of these have been observed in a dynamic HRTEM study of $\mathrm{Cu}$ [94]. Indeed there is no Peach-Koehler force on the step, but it moves because the associated rotation results in a decrease in strain energy. As an alternative to the preceding glide-shuffle, the $h(3)$ TD could move by pure shuffle (see Fig. 23-5 in [62]). However the HRTEM observations rule this out. The $f c c$ case differs from many twin cases in that the successive dislocations have $\mathrm{z}$ components. The strain field is not plane strain. The pure step $h(6)$ in Sect. 3.2.1 is the analog in $h c p$, and also would involve dislocations with $\mathrm{z}$ components. In other cases there can be no pure steps. An example is the $h c p(10 \overline{1} 2)$ twin [100], where the TD Burgers vector is such that there is a residual $\boldsymbol{b}$ no matter what the step height is: in other words there is no O-lattice. Nevertheless, the ratio $b / H$ can be so small that the defect is tantamount to a pure step. An analogous situation exists for large growth ledges in phase transformations [93].

These examples illustrate the dual nature of disconnections. Locally, they can be regarded as the superposition of single defects. However globally they correspond to net Burgers vectors accompanied by shuffles, and produce fields given by the net defect. Large step height disconnections, some corresponding to zonal dislocations, have been observed and/or found in simulations for twins in many metals [59, 62, 101-103]. 

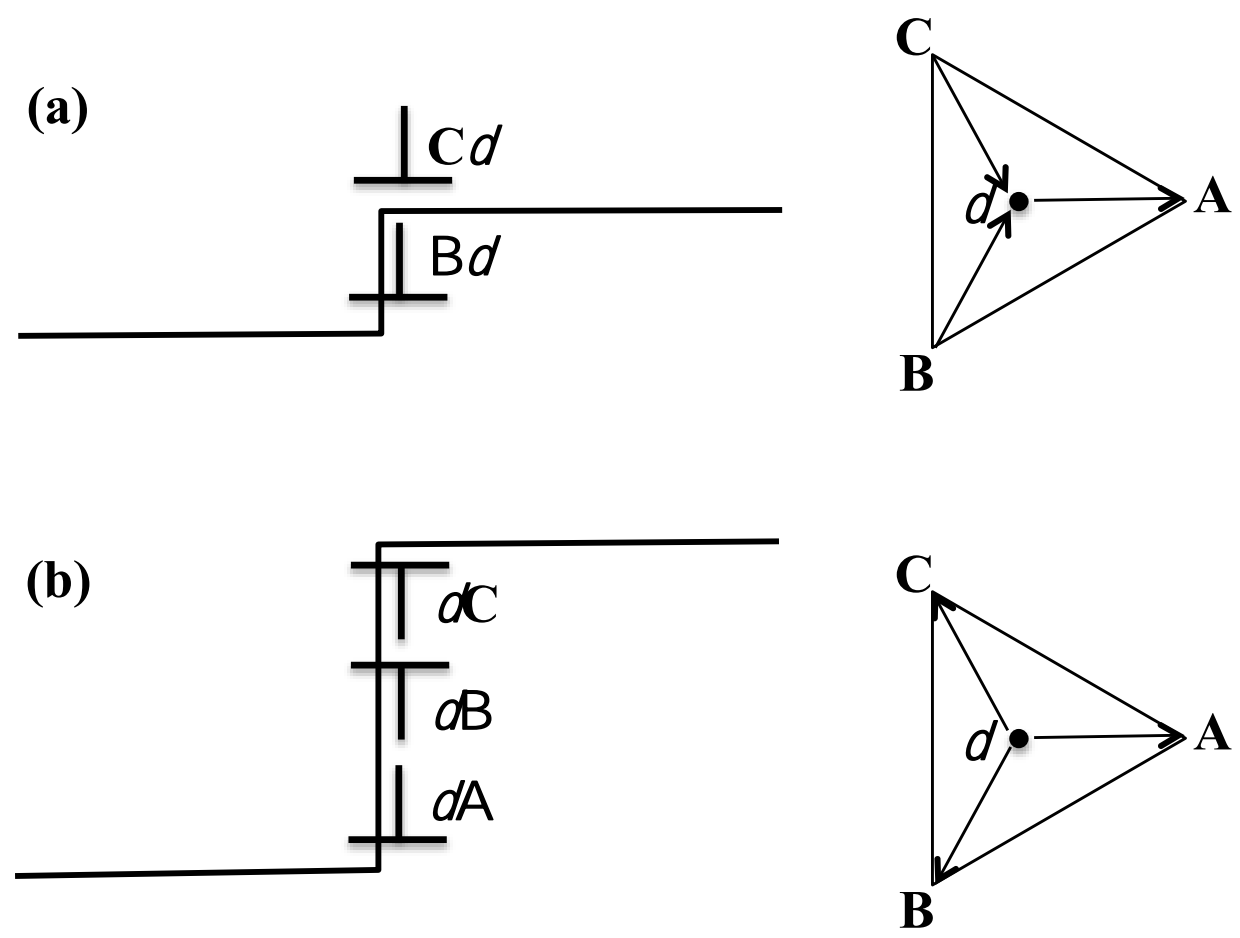

Figure 14. (a) Partial bounding an extrinsic fault in $f c c$. (b) Pure $h(3)$ step in $f c c$.

\section{Motion of unit disconnections}

\subsection{Preliminary remarks}

In this section we discuss the motion of unit disconnections and pure steps. Different aspects of such motion are treated as case studies. These include e-cells that reveal shuffles that entail exchanges of basis pairs, shown for $\boldsymbol{b}(2,2)$ perfect disconnections in $h c p(10 \overline{1} 2)$ twins; types of disconnections, and SDCs in coherent $h c p$ $\mathrm{P} / \mathrm{B}$ interfaces; and observations of disconnection interactions at a P/B boundary.

\subsection{Exchange cells.}

Exchange cells (e-cells) as in Figure 3 are defined in the CDC for a particular unit disconnection [104]. In the $\lambda$ crystal, this cell is defined by the two shortest coincident translation vectors in the coherent terrace plane, together with, for perfect defects, the out-of-plane translation vector, $\boldsymbol{t}(\lambda)$ : similarly, for the $\mu$ crystal, the third vector is $\boldsymbol{t}(\mu)$. For imperfect disconnections, the out-of-plane vectors are $\boldsymbol{w}(\lambda)$ and $\boldsymbol{w}(\mu)$. E-cells 
contain $k$ atoms, where the integer $k$ is the product of the number of planes, $m$ or $q$, and the basis number. This number is 4 for the e-cell in Figure 3 and 1 for the e-cell in Figure 4, and 3 for the e-cell in Figure 5. The cells have physical implications additional to defining $\boldsymbol{b}$ and $h$. For a-unit disconnection advancing by kink-pair nucleation and lateral motion, the cell represents the repeat increment of transformation. For disconnections advancing uniformly under damping control the $y$ and $z$ components define a repeat increment of periodic length along the line of the defect.

In examining e-cells and deciding on Burgers vectors and shuffle vectors, there are some qualitative guidelines, suggested in earlier work [32, 58, 105-109]. The criteria are

-minimum possible $\boldsymbol{b}$ to minimize elastic energy and the Peierls barrier.

-minimum shuffle lengths to minimize activation energy and possible entropic contributions.

When the same displacement could be accomplished by a shuffle or a dislocation, the former would be favored from the elastic energy criterion, but a pair of dislocations could be favored because only half as many "bonds" are distorted by the displacement. Hence there is a trade-off.

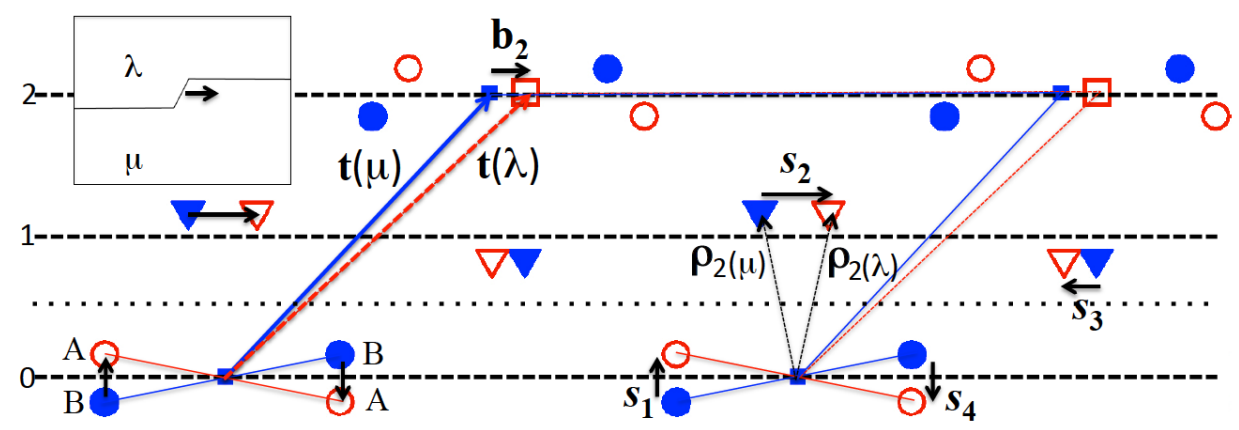

Figure 15. E-cell revealing displacements accompanying TD motion at a (10 $\overline{1} 2)$ terrace. Displacements in layers 0 and 1 are shuffle vectors $\boldsymbol{s}$. 
The (1012) e-cell in Figure 3 can be used to represent shuffles and other displacements accompanying the motion of a disconnection, as illustrated in Figure 15. There, the e-cell corners are fixed on center-of-symmetry (COS) sites. In some cases it is clearer to fix the corners on coincident atom sites. As shown in the box the twin $\lambda$ is propagating downward by the motion of a TD with a + step to the right. Hence, with the convention (B1) in Appendix B, the dividing surface is between planes 1 and 2. The step height is $h(2)$ and $\boldsymbol{b}$ from Eq. (2) is as shown. The Burgers vector is, approximately, $\boldsymbol{b} \cong$ $1 / 12$ [10 111$]$. In the transformation of the cell, the accompanying displacements can be envisioned as being mapped from the CDC to the ideal bicrystal. A simulation for $\mathrm{Zr}$ [100] indicates that $\boldsymbol{p}$ is zero for this twin so the position vectors, shown for one pair of atoms in Figure 15 as dashed lines, are $\boldsymbol{u}^{0}$ as defined in Appendix B. Then, the shuffle vectors are given, in accord with Eq. (B4), by

$$
\boldsymbol{s}=\boldsymbol{u}^{\mathbf{0}}(\lambda)-\boldsymbol{u}^{\mathbf{0}}(\mu)
$$

The lower index on $\boldsymbol{u}$ corresponds to the number of the shuffle. The shuffle vectors according with Eq. (13) are shown in the figure.

The depiction in Figure 15 can be thought of as the ideal bicrystal with embedded markers as mentioned previously. In relaxing to the real bicrystal, added transient displacements $\boldsymbol{u}^{\prime}$ appear near the interface and are revealed in atomistic simulations. In that sense, the e-cell can be imagined to correspond to superposing cells of $\mu$ atoms and $\lambda$ atom, both far from the interface where local strains are negligible [105]. One could then simulate the transition by moving an interface from such a $\mu$ cell to such a $\lambda$ cell by successive disconnection motion. This process was observed in dynamic simulations in Zr [105], where a many-body empirical potential [110] was employed. The simulations allowed detailed trajectories to be followed. In the region near the interface, the cells entered the interfacial distortion field, and underwent large displacements. That is, local displacements, including a Gibbs excess volume per unit area $\delta_{e}$, with magnitude $\sim 0.01 \mathrm{~nm}$, are actually distributed over a finite region near the interface. The observed atom movements verified a specific shuffle mechanism for the first time: the shuffles were those depicted in Figure 15. 
The shuffles in plane 0 are general shuffles, while those in plane 1 are glide shuffles [62]. The basis pairs are labeled A (left) and B (right) in Figure 10 and can be viewed as superposed lattices of an A set and a B set. All perfect Burgers vectors correspond to A-A or B-B vectors.

In order to conserve atom sites, the two shifts must balance, condition (14):

- for inter-basis exchanges, the A-B and B-A exchanges must be numerically equal.

Shuffle vectors need not be translation vectors in the CDC and need not be A-A or B-B vectors. The shuffles $\boldsymbol{s}_{1}$ and $\boldsymbol{s}_{4}$ are B-B and A-A shuffles, respectively, of the same basis pair. If the A-A type shuffles were selected on level 1, the shuffle vectors would be large. Instead, the observed, shorter A-B, $\boldsymbol{s}_{3}$, and B-A, $\boldsymbol{s}_{2}$, shuffle vectors are favored by criterion (12). These A-B shifts are called swaps in [105].

Analogous cells have been suggested earlier [58, 105]. However, in those models, the Burgers vector is implicitly smeared into a continuous distribution over the cell in those models. Thus, the assignment of discrete physical values for the displacement accompanying dislocation motion cannot be done precisely in the earlier models.

Motion of the $h(2)$ dislocations in this case are favored by tensile loading [111]. Shear in the opposite sense with TDs of opposite sign $\boldsymbol{b}$ entails a large barrier because of the associated high energy fault $[14,103]$ and is never observed.

\subsection{Effects associated with $p$}

There can be large shifts $\boldsymbol{p}$ related to relative atom positions in the CDC or e-cell, and small, often vestigial, shifts associated with nonlinear relaxations at the interface. In general, the description of likely shuffles and other vectors can be determined by e-cells where the small nonlinear shifts are neglected. The latter then can be added at the end of the analysis if significant. The (301) $f c c$ twin illustrates the effects of large shifts $\boldsymbol{p}$. The

highest point symmetry e-cell, determined as in Figure 5, is shown as Figure 16(a), and displays the Burgers vector $\boldsymbol{b}(2)=\boldsymbol{b}$. The conventional in-plane shuffle vector is shown as $\boldsymbol{s}^{\prime}{ }_{1}$. However, this vector is large, $0.89 a_{0}$, where $a_{0}$ is the [100] unit cell dimension. Hence, it is energetically favorable, in accord with criterion (12), for the shuffle vectors 
to entail out-of-plane components, shown as $\boldsymbol{s}_{1}$ and $\boldsymbol{s}_{2}$. The atoms in the plane offset by $1 / 2[00 \overline{1}]$ are shown as triangles. The magnitude of these shorter vectors is $0.57 a_{0}$. The shuffles are equal and opposite as indicated: they are glide shuffles and can be regarded as being composed of a screw dislocation dipole. At an initially planar (001) free surface or fiducial marker, the occurrence of these shuffles would be manifested by a surface rumpling of rows of atoms.
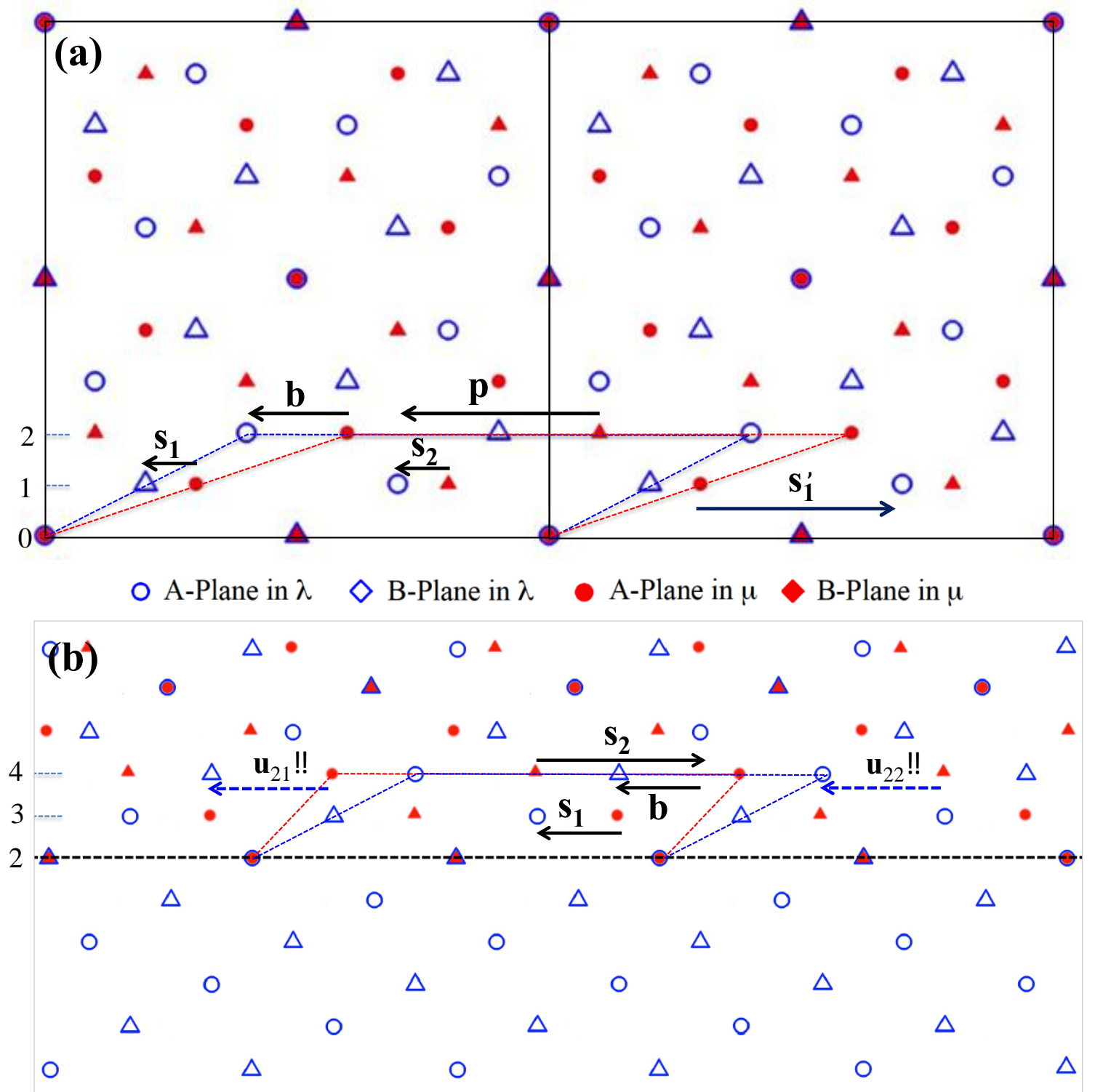

Figure 16. Two unit cells of the CDC for a $\Sigma 5$ twin in $f c c$. Unit cell dimensions are 1/2[031] in the y direction and $1 / 2[0 \overline{1} 3]$ in the $\mathrm{z}$ direction. Here $\boldsymbol{b}(2)=\boldsymbol{b}=-1 / 10[0 \overline{3} \overline{1}]$ and $h(2)=$

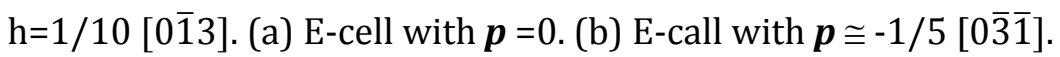


These twins were studied in an atomistic simulation of $\mathrm{Al}$ [70,71]. These revealed that the CDC was shifted by $\boldsymbol{p} \cong-1 / 5[0 \overline{3} \overline{1}]$, as shown in Figure 16(a). In general $\boldsymbol{p}$ need not be rational, but the preference for symmetry in crystals. The Bloch theorem suggests that a rational vector, or one near it, is not unlikely. The essential reason for this shift is the close approach of second nearest-neighbour atoms across the interface in Figure 16(a). This $\boldsymbol{p}$ vector is nearly a DSC vector [56] and shifts the structure of plane 0 so that is the same as that in plane 2, i.e., that shown as the new composition plane 2 ' in Figure 16(b). Indeed, the ideal bicrystals in Figure 16 correspond to Figures 4(b) and 4(e) in $[70,71]$, the predecessor of the topological theory [1]. The Burgers vector is unchanged, but there is now a different shuffle vector, $\boldsymbol{s}_{1}$ which is given by the extension of Eq. (13).

$$
\boldsymbol{s}=\boldsymbol{u}(\lambda)-u(\mu)=\boldsymbol{u}^{\mathbf{0}}(\lambda)-\boldsymbol{u}^{0}(\mu)-\boldsymbol{p}
$$

The shift results in shorter lengths for these vectors, so the out-of-plane shuffles are no longer favored. Thus, not only is the terrace lower in energy for the shifted TD, but the activation energy for motion should be less according to criterion (12). This example shows the importance of rigid displacements in establishing the probable shuffle vectors for a given type of disconnection. Also shown in Figure 16(b) are possible synchroshear displacements (see Sect. 7). The overall displacements are less for synchroshear because of the large vector $\boldsymbol{s}_{2}$ for the conventional defect. Other disconnections can be more stable in the shifted structure. For Figure 16(b), criterion 12 shows that $h(1)$ and $h(4)$ defects are more likely while $h(3)$ are less so. For larger $\boldsymbol{p}$ disconnections with opposite sign could be more stable, i.e., those represented in Figure 16(a) with step heights $h(6)$ or larger. This diagram shows the value of the dual CDC/ideal bicrystal representation in the displaced e-cell, emphasized in Figure 16(b) by the absence of solid atoms in the transformed twin, but indicated by the embedded $\lambda$ sites in the matrix. The displaced ecell makes it easier to deduce possible alternate defects. The trend is consistent with the procedure of the topological theory [1]: begin with the highest symmetry CDP, then the highest symmetry CDC, Figure 16(a), then operations that successively reduce symmetry, here Figure 16(b). 


\subsection{Thermodynamic driving force}

For perfect dislocation motion the dominant thermodynamic force is the PeachKoehler force. For TD motion, stresses tend to be large and a second term, quadratic in stress becomes important. Together the force in the $\mathrm{y}$ direction per unit length in the $\mathrm{x}$ direction, acting on a defect transforming from $\mu$ to $\lambda$ is

$$
\mathrm{F} / \mathrm{L}=\boldsymbol{\sigma} \bullet \mathbf{b} \times \boldsymbol{\xi}+[E(\mu) V(\mu)-E(\lambda) V(\lambda)] \boldsymbol{j}
$$

Here $E$ is the strain energy per unit length and $V$ is the unit e-cell volume per atomic length in the $x$ direction. When the disconnection has a $\boldsymbol{b}$ component, the first term is generally larger. However, the second term can be important, and is the only term present for a pure step. In general, the difference in strain energy can arise from the partitioning of coherency strain to thinner crystals or anisotropically softer crystals: this could occur for P/B interfaces (those between prism and basal planes), for example. For twin interfaces, there is no coherency strain, but a subtler effect can arise. If the principal stress coordinates are inclined to the twin plane in an anisotropic elastic model, the stresses in coordinates fixed on the twin plane will differ for the two crystals and a driving force will arise. Motion of pure steps caused by such an effect has been observed using in situ HRTEM studies of $\mathrm{Cu}$ [97, 112].

When rotational partitioning is present, as for the $\mathrm{P} / \mathrm{B}$ case, the coordinates in Eq. (16) are the rotated coordinates of Figure D1 and the pertinent Burgers vector is the GBD vector $\boldsymbol{b}_{\mathrm{g}}$ of the rotated RCDC.

\subsection{The $\mathrm{P} / \mathrm{B}$ interface}

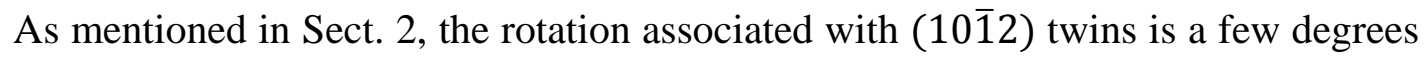
about $[\overline{1} 2 \overline{1} 0], 3.7^{\circ}$ for Mg, Fig. 17. This leaves the (0001) basal plane of one crystal rotated with respect to the $(1 \overline{1} 00)$ prism plane of the other by $87.3^{\circ}$. If two $h c p$ crystals are rotated by $\theta=90^{\circ}$ about [1 $\left.2 \overline{1} 0\right]$, the (0001) basal plane of one crystal becomes parallel to the $(1 \overline{1} 00)$ prism plane of the other, the P/B interface [37]. Thus, the misorientation differs only slightly from that of the twin. With an ideal c/a ratio, this plane would be coherent without misfit. For actual c/a ratios, coherency is created parallel to $(10 \overline{1} 0)_{\lambda} /(0001)_{\mu}$ planes when the $\lambda$ lattice undergoes homogeneous linear expansion, and the $\mu$ contracts. A HRTEM view of a P/B interface, in $\mathrm{Mg}$, containing a 
disconnection is shown in Figure 18 [113]. There have been similar observations for $h c p$ metals, e.g. $[114,115]$. P/B interfaces have been observed widely, and TDs have been discussed in these works $[15,16,36-38,113-117]$. They differ from the present work in the details of CDCs, TDCs, and e-cells, and in the analysis of components of displacement vectors. The ideas in $[116,117]$ are qualitatively correct and represent the first description of $\mathrm{P} / \mathrm{B}$ defects in terms of disconnections. However, the natural dichromatic pattern is used instead of the CDP, so Burgers vectors are not precise.

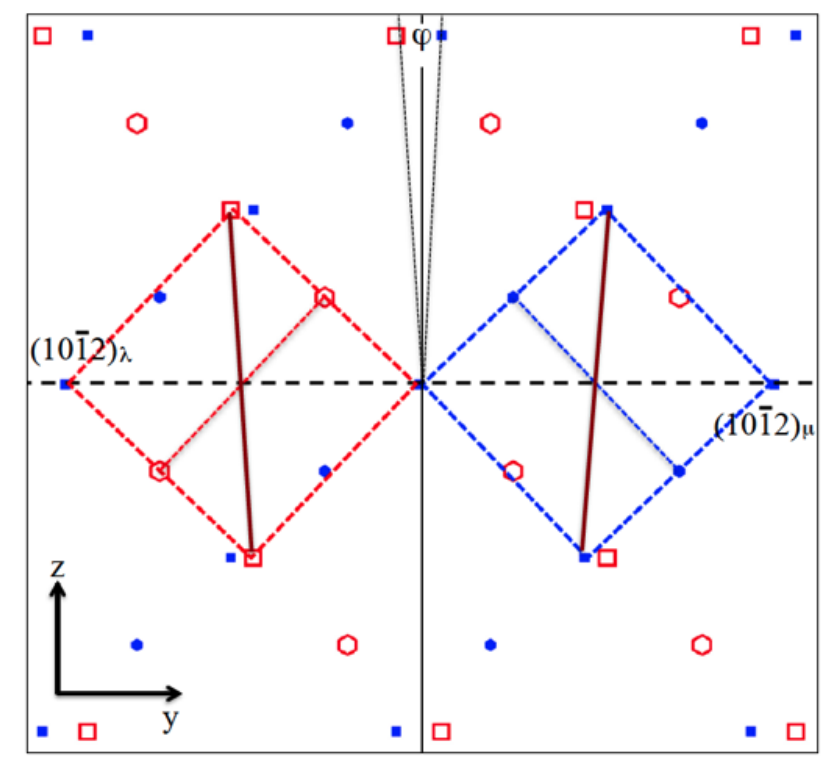

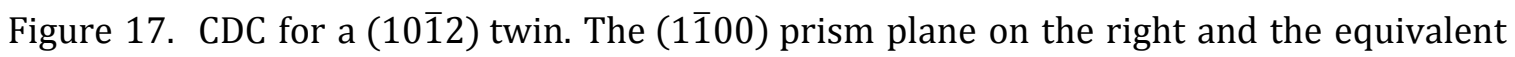
(1100) on the left are shown as solid lines. Both transposed to the center with rotation angle $\varphi$ shown.

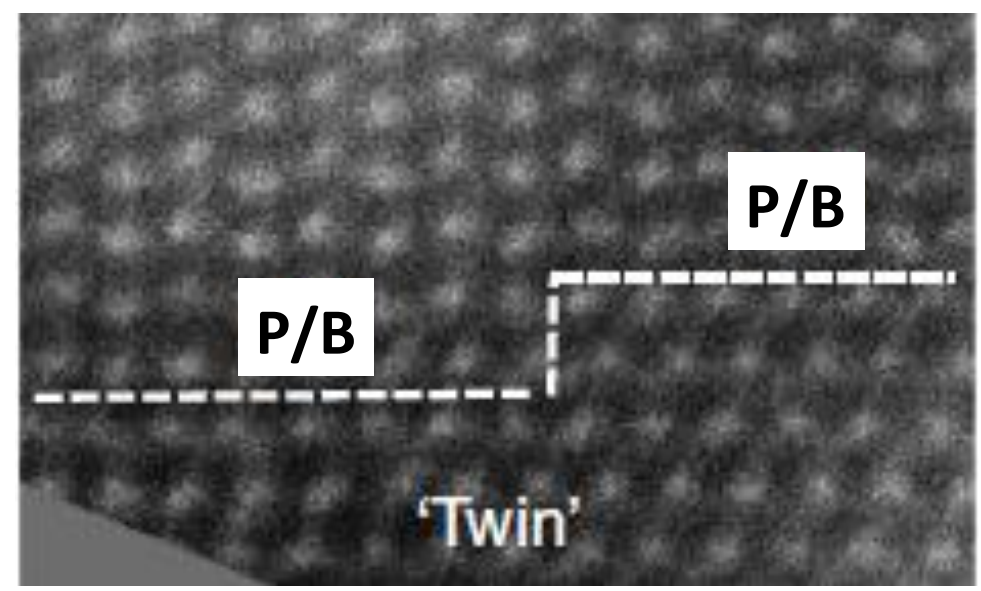

Figure 18. HRTEM image of a disconnection at a P/B interface, $\mathrm{P}$ above and $\mathrm{B}$ below [113]. 


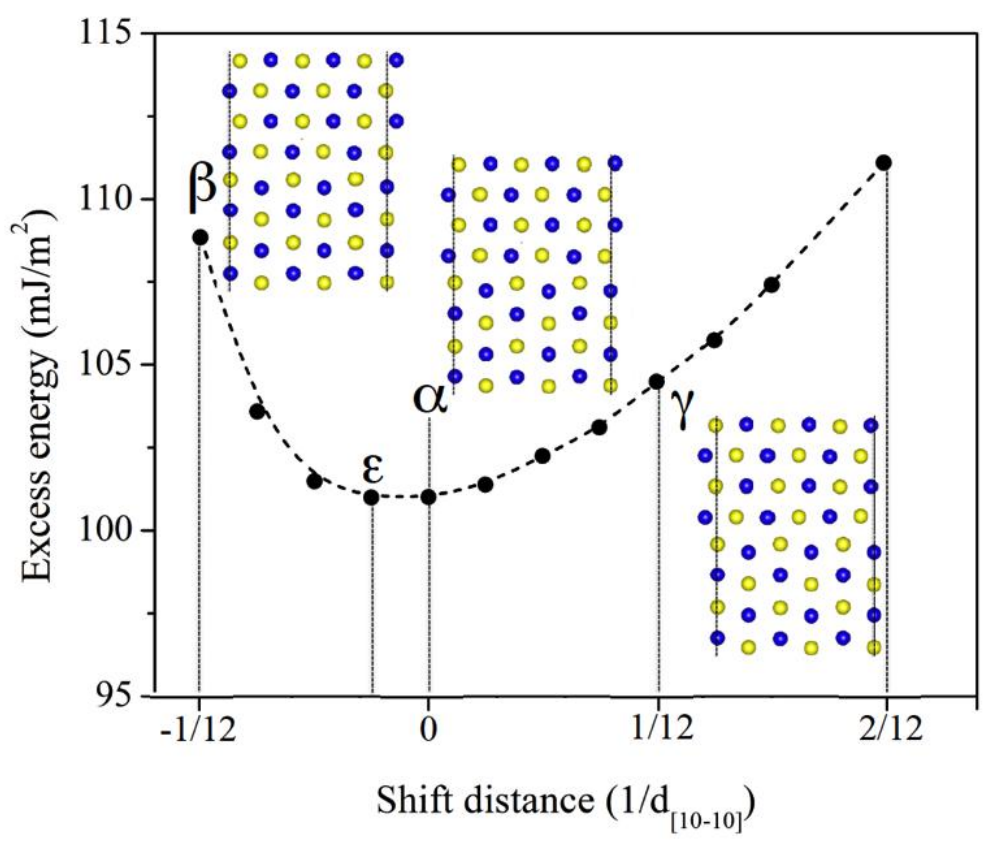

Figure 19. P/B interfacial energy in $\mathrm{Mg}$ as a function of the shift vector $\boldsymbol{p}$ defined in Fig.20.

We have performed first-principle DFT (VASP) calculations giving the interface energy as a function of the shift vector shown in Figure 19. The corresponding alignment $\varepsilon$ at the lowest energy is that shown in Figure 8, close to the $\alpha$ structure in Figure 20(a). The $\alpha$ structure in Figure 20(a) has COS sites in $\lambda$ (square) aligned with the midpoint of an atom pair aligned in the z-direction in a (0001) plane, also shown as a square. With an ideal c/a ration the separation of both pairs would be identical. The maximum symmetry structure, $\gamma$, would have COS sites aligned in the z-direction, so formally the $\alpha$ structure would be transposed by $\boldsymbol{p}$ relative to $\gamma$ and the corresponding complex would be a transposed TCDC. However, the $\alpha$ structure has an anti-mirror glide plane normal to $y$ as is evident from the vertical planes demarcated in Figure 20, and this symmetry leads to the characterization described next.

The e-cell in Figure 20(a) shows that the likely disconnection has $h(2)$ and $\boldsymbol{b}(2)=$ $\boldsymbol{b}=\left[\begin{array}{lll}0 & 0 & b_{z}\end{array}\right]$, with four atoms per unit e-cell. For clarity the e-cell is fixed on coincident atom sites. The next shortest vectors would have added in-terrace-plane components $1 / 3[10 \overline{1} 0]$ or $1 / 3[11 \overline{2} 0]$, both with substantial elastic energies, so that both are unlikely. The e-cell shows that the interface is moved downward by either a disconnection with a 
step moving to the right or one with a step moving left. The rest of the transformation entails four relatively short shuffle vectors. The energy minimum and the excess volume $\delta_{\mathrm{e}}$, Table1, are roughly consistent with bond stretching effects.

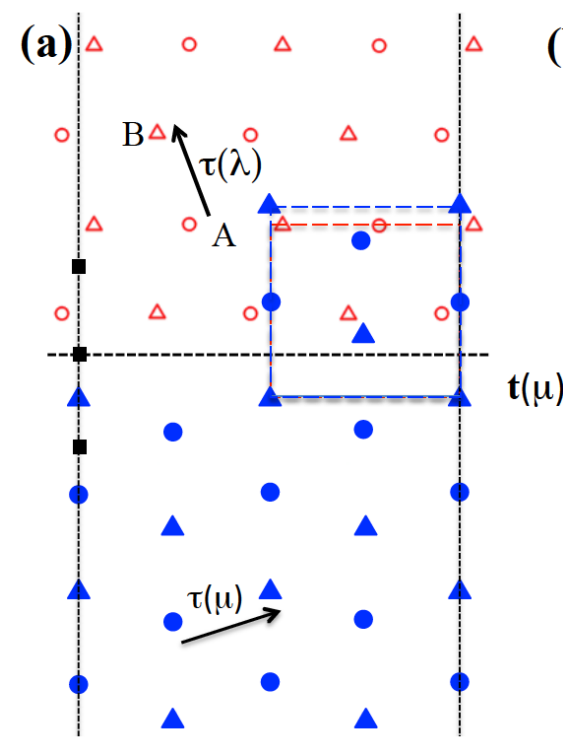

(b)

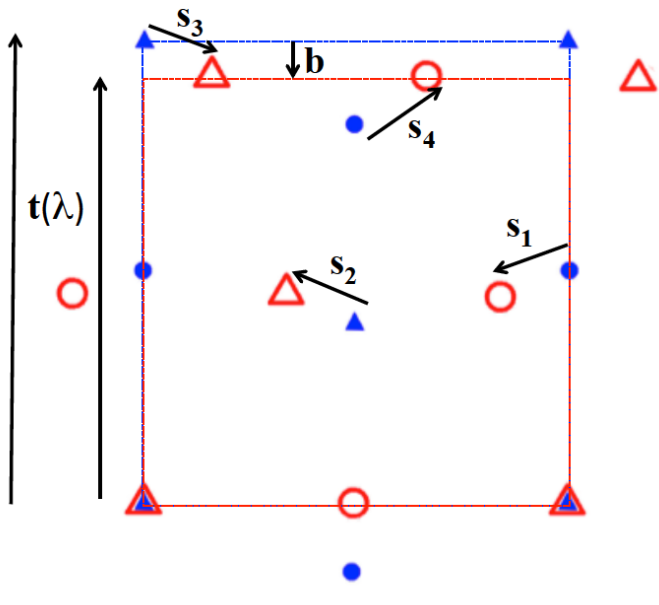

Figure 20. (a) Ideal bicrystal for a P/B interface in the $\alpha$ structure, with the commensurate plane, dotted, corresponding to $(10 \overline{1} 2)_{\lambda} /(1 \overline{1} 00]_{\mu}$. Interface shown by dashed line. A COS site in $\lambda$ and an intermediate site in $\mu$ are shown as squares. The vectors, $\tau$, connecting A-B basis pairs, are denoted. The E-cell with the origin transposed to an atom position in $\mu$ is shown by solid lines. (b) E-cell with displacements shown. The origin $\boldsymbol{p}=0$ for the shift vector $\boldsymbol{p}$ used in Fig. 19 is defined with the $\alpha$ structure as a reference.

Table 1. Values of excess volume for P/B interfaces in several metals.

\begin{tabular}{|c|c|c|c|c|c|c|}
\hline Metal & $\mathrm{Cd}$ & $\mathrm{Zn}$ & $\mathrm{Mg}$ & $\mathrm{Zr}$ & $\mathrm{Ti}$ & $\mathrm{Be}$ \\
\hline $\mathrm{P}_{\mathrm{z}}$ or $\delta_{\mathrm{e}}(\mathrm{nm})$ & -0.003 & -0.008 & 0.002 & -0.004 & -0.001 & 0.005 \\
\hline
\end{tabular}

Simulations reveal that $h(1)$ TDs can be present. The e-cell for such a defect is shown in Figure 21, including views in both the $x$ and $z$ directions.

The terrace that is created is degenerate with the original terrace but rotated by $\pi$ about the $\mathrm{z}$ axis. Hence the vectors defining the Burgers vectors are $\boldsymbol{w}$ vectors, Appendix 
$\mathrm{B}$, and the defect is a domain disconnection. A succeeding domain disconnection would have the same $\mathrm{z}$ components of displacement but opposite sign y components, Figure 20(a), and would restore the original terrace, Hence the pair of domain disconnections are equivalent to one perfect disconnection.
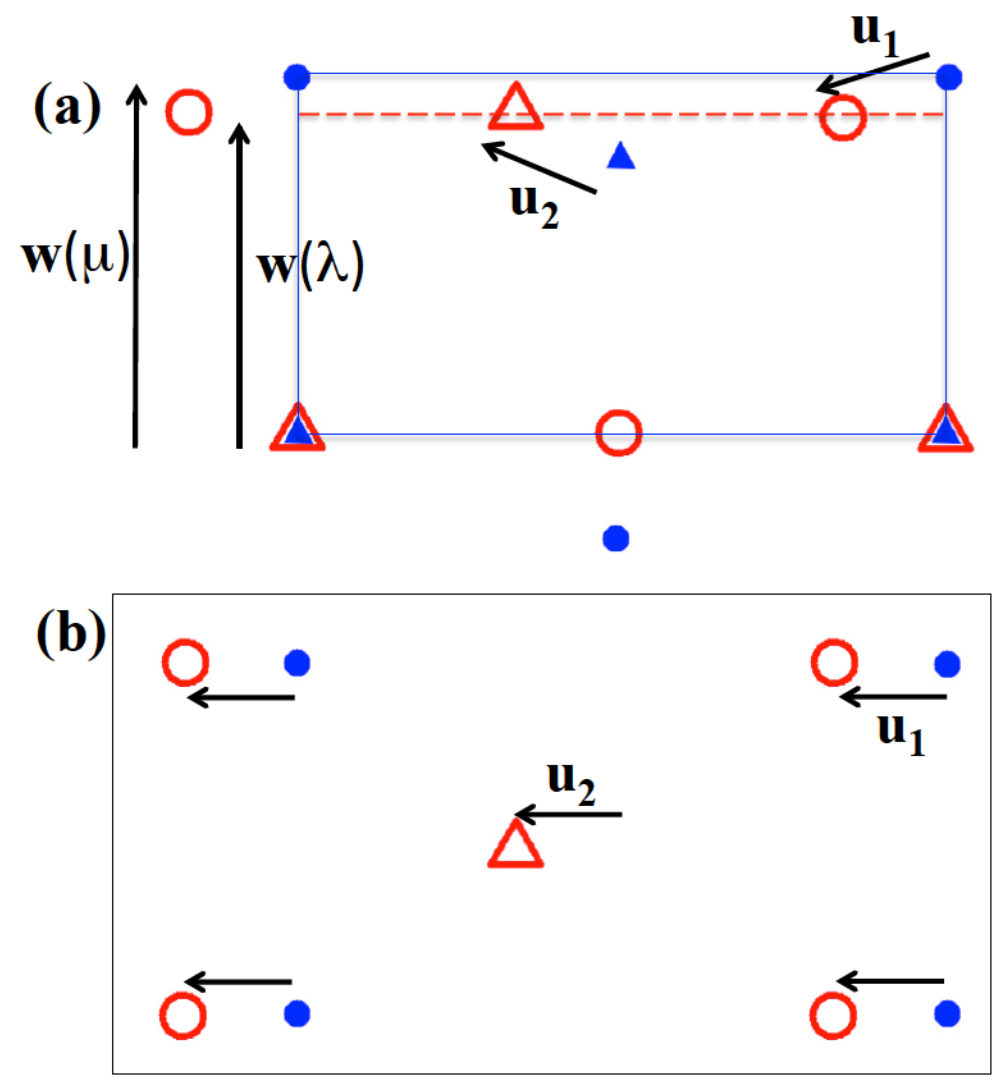

Figure 21. E-cell for $h(1)$ domain disconnection.(a) View normal to $x$. (b) View normal to z.

There are two possible structures for the domain disconnections, with different interpretations of the $\boldsymbol{u}$ vectors in Figure 21. In the first, the displacements other than the $b_{z}$ components are pure shuffle vectors and $\boldsymbol{u}_{1}$ and $\boldsymbol{u}_{2}$ become $\boldsymbol{s}_{1}$ and $\boldsymbol{s}_{2}$, respectively. In the second $\boldsymbol{u}_{2}$ remains $\boldsymbol{s}_{2}$, but the $y$ component $\boldsymbol{u}_{2}$ also becomes a dislocation component $\boldsymbol{b}_{\mathrm{y}}$. Thus the dislocation $\boldsymbol{b}_{2}$ has both a $z$ component $\boldsymbol{b}_{\mathrm{z}}$ and a glide component $\boldsymbol{b}_{\mathrm{y}}$. For this case $\boldsymbol{b}_{\mathrm{y}}=\boldsymbol{\tau}(\lambda) / 2$, where $\tau(\lambda)$ is the vector connecting a basis pair in $\lambda$, Figure 20(a). In either case the result of the pair of domain defects is the same. Which of these is preferred would depend on the trade-off in criterion (12) and could only be resolved by a dynamic atomistic simulation. 
Results of simulations of $\mathrm{Zr}$ [105] using a semi-empirical potential [110] indicate, in contrast to $\mathrm{Mg}$, that the $\gamma$ structure has minimum energy. The density functional theory (DFT) method used for our work on $\mathrm{Mg}, \mathrm{Ti}$, and $\mathrm{Zr}$ here is a first-principles method [118] and should generally be more accurate. Yet, the energy differences in Figure 19 are small, so possibly the real equilibrium structure could be somewhere in the range between $\beta$ and $\gamma$.

Motion of these $\mathrm{P} / \mathrm{B}$ disconnections along the interface causes a volume change, as reflected by the finite component of their Burgers vector normal to the interface, $b_{z}$, Figure 18. Nevertheless, the motion of these P/B defects remains conservative, as can be confirmed by substitution into the equation $[6,49]$ for the diffusive flux accompanying defect motion. Because the mechanisms entail motion on unique terraces, the $\mathrm{z}$ displacements are special in a sense. Burgers vector components $b_{z}$ are spontaneous and require no activation: they arise from the change in $\mathrm{d}$-spacing of the white-black planes. Shuffle components $s_{\mathrm{z}}$ are associated with similar shifts across the interface and again occur spontaneously. Hence, one could in principle suppress the $\mathrm{z}$ displacements, carry out the transformation and then superpose the $z$-displacements.

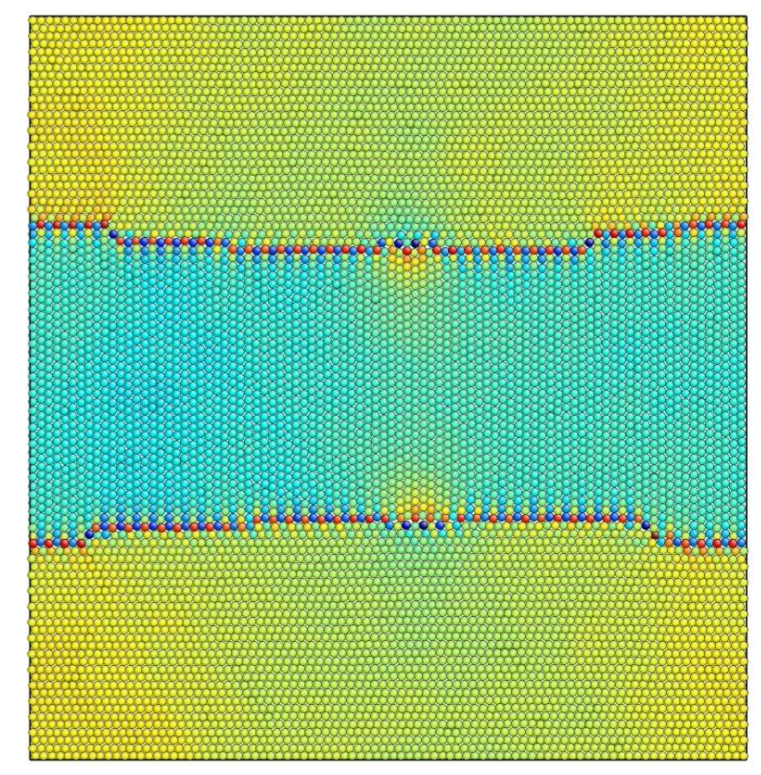

Figure 22. Disconnections nucleated at the interface of a thin film with a P-oriented interface. Atoms are coloured according to their excess energy. 
The P/B interface provides an interesting example of Eq. (16). Figure 22 shows, for $\mathrm{Mg}$, a thin layer with a P oriented interface in a matrix of B orientation. There is no first order Peach-Koehler force on interface disconnections. Yet, the coherency strain is localized in the thin film because of the stiffness of the matrix. Hence there is a driving force associated with the second term in Eq. (16). Shrinkage of the film reduces the total strain energy. As a consequence disconnections spontaneously nucleate at the interface and cause the thin layer to shrink.
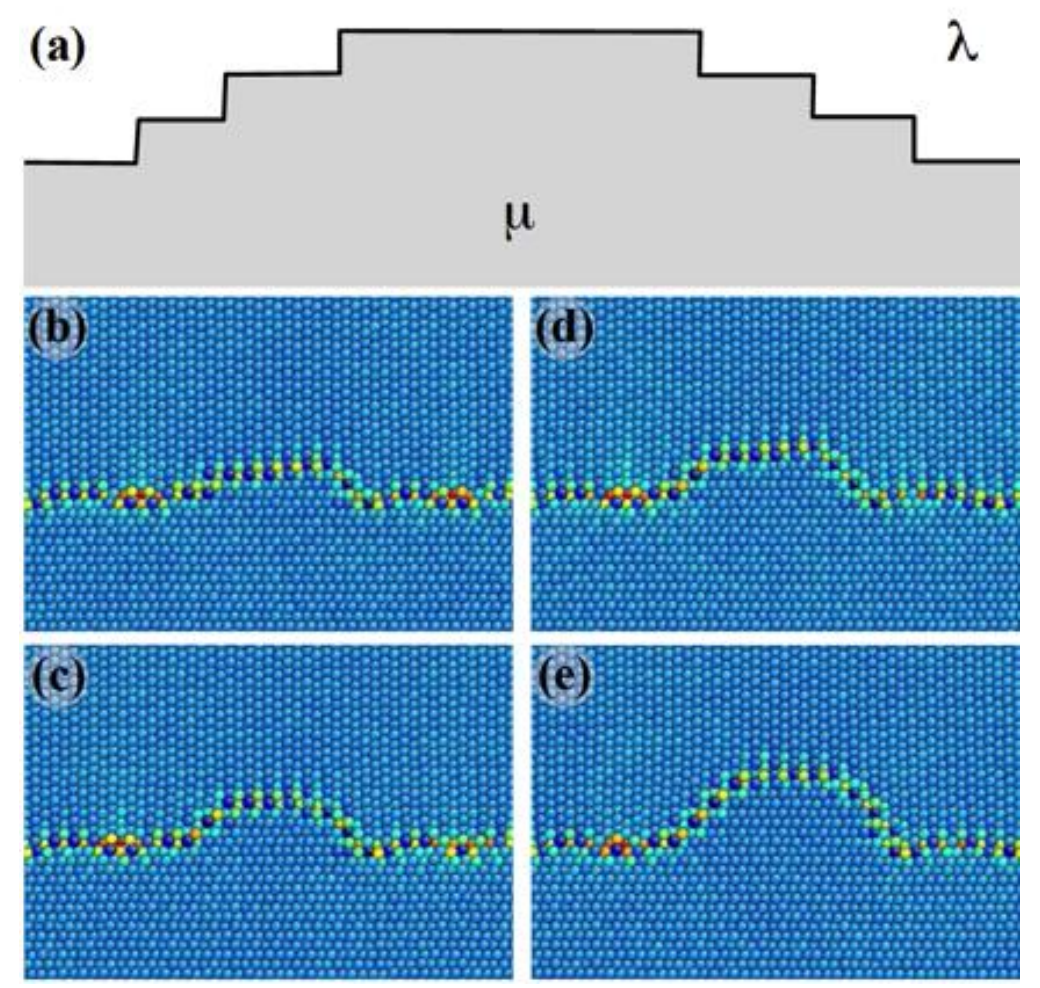

Figure 23. (a) Repeated nucleation of $h(2)$ disconnection pairs at a thin layer interface, prism top, basal bottom [113]. Atoms are coloured according to their excess energy.

\subsection{Arrays and motion of disconnections}

In this section, we present results from molecular dynamics simulations of $h c p \mathrm{Mg}$. Figure 23 shows the $\mathrm{P} / \mathrm{B}$ interface of a thin layer of $\lambda$, with the prism interface, in $\mu$. The stiffness of the $\mu$ forces all of the coherency strain into the $\lambda$. At $10 \mathrm{~K}, h(2)$ disconnection pairs nucleated successively driven by the coherency strain. This effect also occurred under a compressive stress of $1.5 \mathrm{GPa}$ normal to the interface $[37,113]$, with the 
successive pair nucleation shown in Figure 23. In this case the Poisson stress $\sigma_{y y}$ differs in the two crystals because of differing anisotropic elastic constants. In both cases, there is no Peach-Koehler force since $\boldsymbol{b}$ has no y component. The second term in Eq. (16) is present and large because of the strain partitioning. Processes of this type occur in the annealing of nanotwins in $\mathrm{Cu}$, where thin twin layers are selectively removed [112]. They could occur in general for annealing twins, but, because of the typically small driving force, heterogeneous nucleation is more likely [119].
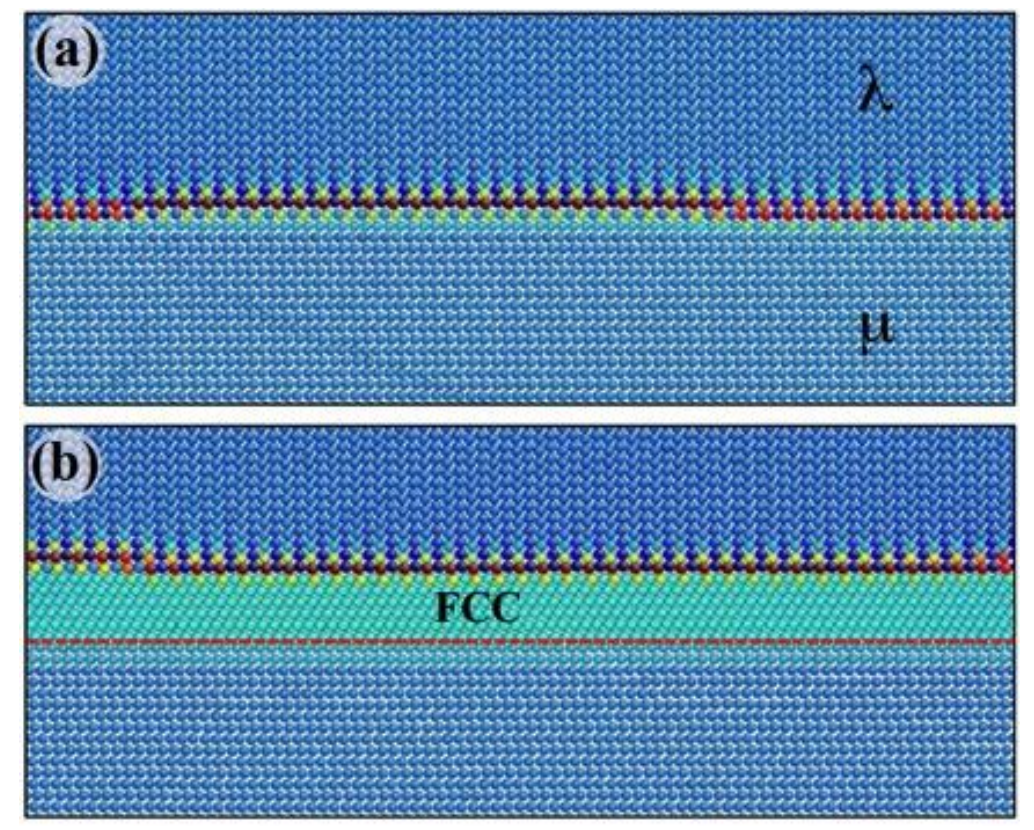

Figure 24. Nucleation of $h(1)$ disconnection pairs, producing a $f_{c c}$ layer. Prism top and basal bottom. Atoms are coloured according to their excess energy.

Figure 24 represents an interface of the same kind under an applied shear stress $\sigma_{y z}$ $=150 \mathrm{MPa}$. In this case the first term in Eq. (16) is present because the $h(1)$ disconnections have a $b_{y}$ component. Consequently the first disconnection nucleates as shown in Figure 24(a). The nucleation of a second pair with the opposite sign $\boldsymbol{b}$ as in Figure 20 cannot occur because the force on the pair would oppose nucleation. Instead, another pair with the same $b_{\mathrm{y}}$ forms, leaving a fault relative to $h c p$. Yet, this fault locally produces a $f c c$ layer. This is possible because of the typically low free energy difference between $h c p$ and $f c c$ phases. Repeated nucleation produces the $f c c$ layer shown in Figure 24(b). The presence of a shear stress does not help P/B propagation. This is obvious for 
$h(2)$ disconnections. For $h(1)$ disconnections with $\boldsymbol{b}$ character, the stress would aid alternate disconnection motion of half the defects but hinder motion for the other half because of the domain line character. Molecular dynamics simulations of motion on a $\mathrm{P} / \mathrm{B}$ interface in $\mathrm{Mg}$ showed that motion was produced by $h(2)$ disconnections [39]. Simulations in $\mathrm{Zr}$ using a semi-empirical potential found motion to be caused by $h(1)$ defects [40]. The consequence of no aid by an applied shear stress applies to both cases. The DFT method should be more accurate, but the issue about the general step height of $\mathrm{P} / \mathrm{B}$ defects remains an open question.

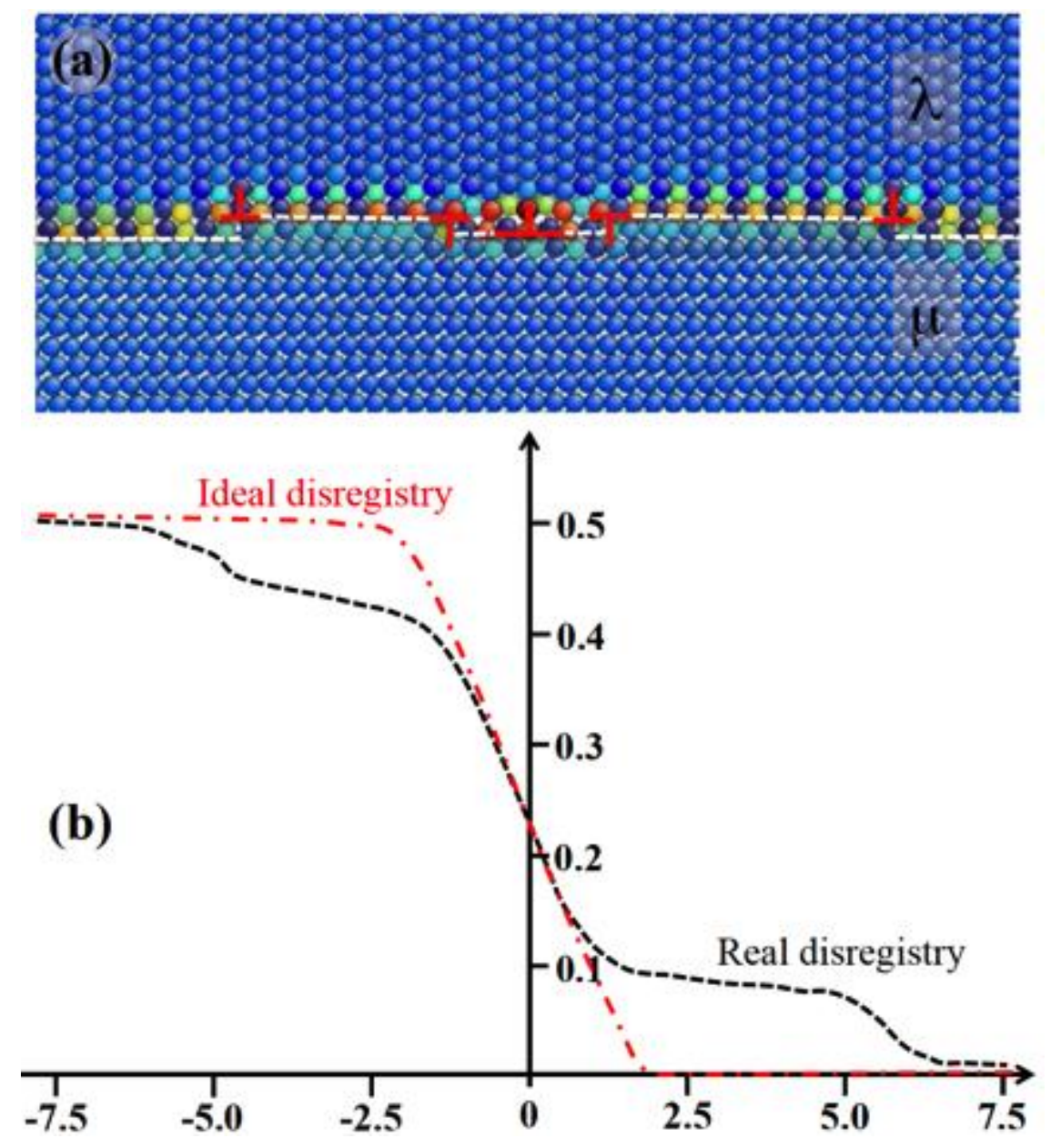

Figure 25. A [0001] $]_{\mu}$ misfit dislocation, with two $h(1)$ disconnection pairs. Atoms are coloured according to their excess energy.

The misfit strain is relatively large, 0.065 for $\mathrm{Mg}$. Strain relief can be provided by misfit edge dislocations [0001] $\mu$ or by mixed $1 / 3[2 \overline{1} \overline{1} 0] \lambda$ dislocations. An example of the 
former is shown in Figure 25(a). The local strain field is so large that two $h(1)$ disconnection pairs nucleated to spread the local misfit strain, indicated by the spreading of disregistry. In Figure 25(b). We anticipated that this defect might split into two synchroshear $1 / 2[0001] \mu$ defects [120], but the disregistry shows that this is not the case.

While the array of misfit dislocation in Figure 25 is a likely outcome of standard nucleation processes, an alternate relaxed interface has been found in a simulation of $\mathrm{Mg}$ [117]. There, misfit was achieved by a regular array of misfit dislocations of two-types with associated relaxations like those in Figure 25. Indeed, a larger step height, alternating up-down set of facets, would lower coherency strain and could be stabilized. In the serrated region the coherency stress would be the average of that in the two crystals and, depending on their relative fractions, could be zero.

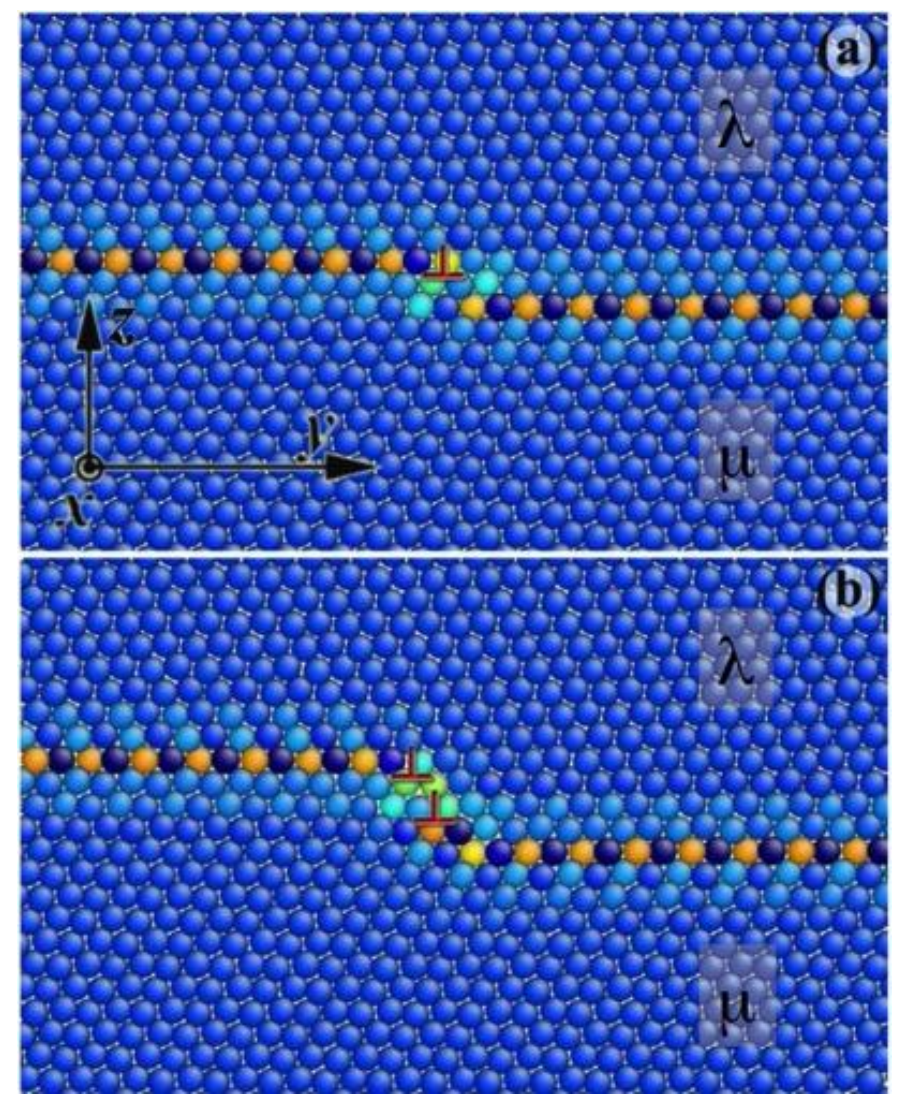

Figure 26. (a) Mixed $h(2)$ disconnection and (b) Edge $h(4)$ disconnection on (1011) twin boundary. Atoms are coloured according to their excess energy. 


\subsection{Combination of disconnections}

Twinning in the hcp pyramidal systems $(\overline{1011)}$ and $(\overline{1013})$ is of interest because it entails two $h(2)$ disconnections with equal and opposite screw components and an $h(4)$ disconnection $[14,38,98,111,121-123]$. We discuss the (1011) twin, which has characteristics analogous to the (1013) twin. The two types of defects are shown in Figure 26. Simulations using both semi-empirical potentials [14, 38, 98, 120] and DFT methods [118] as well as experimental observations [1,14] agree with the early postulate of Westlake [122]. The mobility of the $h(2)$ disconnections is much greater than the $h(4)$ disconnection, but the line energy of the latter is lower. The lower energy arises because the $h(4)$ disconnection is the superposition of two $h(2)$ disconnections whose screw components cancel, leading to a blower elastic energy. If only one $h(2)$ TD with a unique screw component was active, the $\eta_{1}$ direction would differ by $14^{\circ}$ from the observed $\eta_{1} \|[\overline{1} 01 \overline{2}]$, which would be consistent with the edge $\boldsymbol{b}$ of the $h(2)$ disconnection. Thus, the view that emerges $[98,123]$ is that $h(2)$ disconnections with alternating screw components nucleate in an alternating sequence. Such nucleation would also be favored by cancellation of screw components at the TD source. These higher mobility TDs propagate the twin. Once the TDs come to rest, however, they combine to form the lower energy $h(4)$ defects.

Opposite from the (1012) twin, the (1011) and (1013) twins are favored by compressive loading. Motion of the TDs in the opposite direction for the latter pair is very unfavorable, analogous to the situation for the (1012) twin.

\section{Facets and disclinations}

There are now many observations of P/B facets with lengths up to $2-5 \mathrm{~nm}[15,16$, 36-38]. These have been treated in simulations and mechanisms indicated where the facets themselves move by disconnection transit [15]. Molecular dynamic simulations $[39,40,120]$ have verified that P/B facets move by such a mechanism. Facets have been associated qualitatively with disclinations [116], but the details of the model only approximate the present analysis.

Mechanistically, single disconnections with the same sign $b$ and $h$ move in the same direction, and when a single defect is slowed by local impediments, other defects 
accumulate locally. While the defects repel with large separations, there is a short-range attractive interaction between them [92, 93]. Simulations indicate that accumulations into a tilt-configuration facet should occur up to two or three nanometers [93, 94], including the facet at the tip of a lenticular shaped twin tip [124, 125]. In cases such as a $\mathrm{P} / \mathrm{B}$ facet on a (1012) terrace, the formation of a coherent, low-interfacial energy, facet structure provides additional driving force for faceting and even larger facets can be favored. We select this $\mathrm{P} / \mathrm{B}$ facet in $\mathrm{Mg}$ as an example.

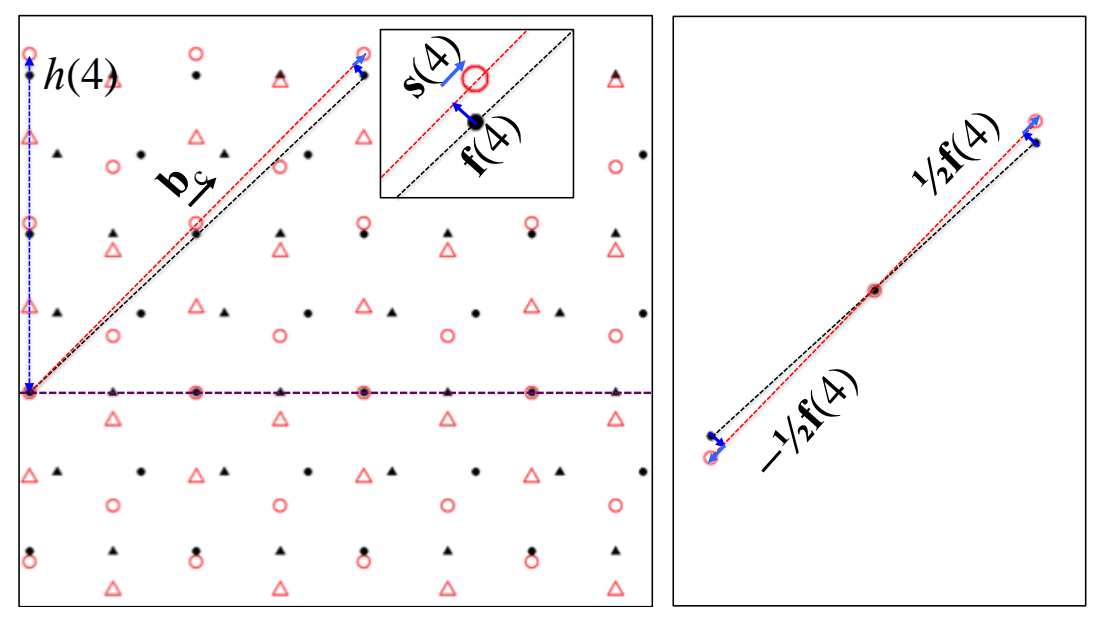

Figure 27. Facets on a $h c p(10 \overline{1} 2)$ twin plane in $\mathrm{Mg}$, described as an $h(4,4)$ disclination, Partitioning of $\boldsymbol{f}$ shown at side.

The $\mathrm{CDC}$ for this case is that for the $\mathrm{P} / \mathrm{B}, \alpha$ coincident reference structure. As discussed in Sect. 3.3.4, the P/B facet has disclination dipole character with an associated rotation angle of $\Delta \varphi=3.7^{\circ}$ for $\mathrm{Mg}$. A facet with the $\mathrm{P} / \mathrm{B}$ structure is analyzed as a disclination with $h(4,4)$ in Figure 27. The total displacement for step, $\boldsymbol{u}=\boldsymbol{u}(4,4)$, is shown and its three components are shown in the inset. The disclination dipole Frank vector $f$ [81] produces a local stress field given by Eq. (6) [62]. Significantly, the stresses of Eq. (6) diverge at both ends of the facet (at $y=0$, and $z=0$ or $-d$ in coordinates fixed on the facet). As a consequence the rotation must be partitioned as indicated in Figure 27: to our knowledge this necessity has not been previously made explicit. The partitioned disclination is effectively two disclinations with the same $\Delta \varphi$ meeting at a coincident midpoint that is stress free by condition (8). Additionally, the Frank vector is partitioned 
into two stress sources with vectors $\pm f / 2$ at the singularities in accord with Figure 10 . The remaining terms are a shuffle $s(4)$ and coherency stresses $b_{c}$ associated with the removal of the misfit caused by $s(4)$. If the structure were instead $\beta$ or $\gamma$, a shift by $\boldsymbol{p}$ would occur and another added shuffle $s$ would be present. If a down step occurred, it would unlikely be a reentrant version of Figure 27 because of the added interface. Instead a step slanted in the other sense would be more likely. The defect would have different vector properties except for $b$.
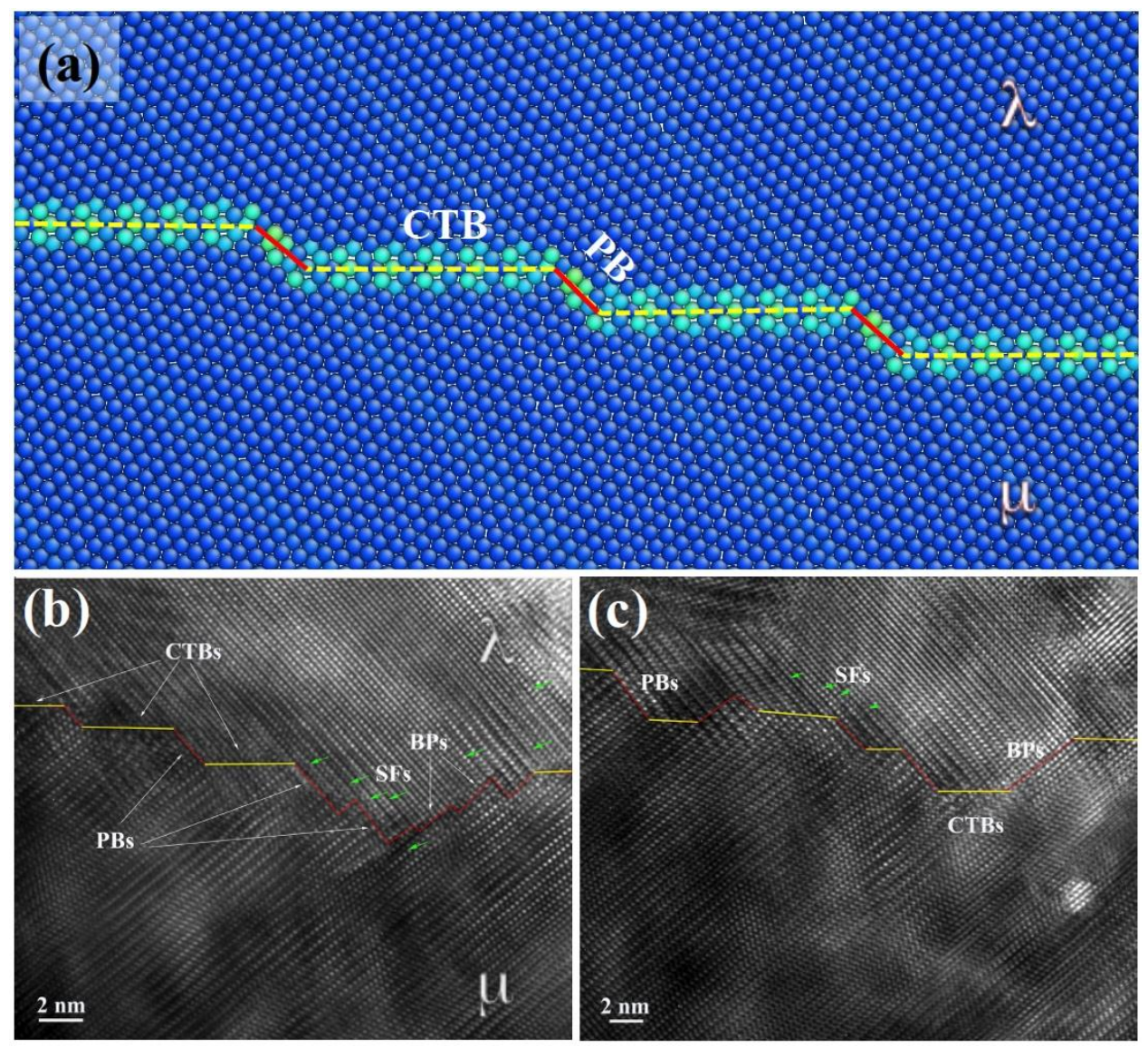

Figure 28. (a) Simulated coherent (1012) twin plane with PB facets. (b) and (c) HRTEM images show PB facets with different heights in Co [115]. Atoms are coloured according to their excess energy.

Figures 28 and 29 show facets in $\mathrm{Co}$ and $\mathrm{Cu}$, respectively [112, 115, 126]. We see that facets can correspond to incoherent twin boundaries. In special cases they could correspond to coherent twin boundaries, usually when the twin boundary terminates at 
two free surfaces. Structures like that in Figure 29(a) are observed for annealing twins at orders of magnitude larger size scales.
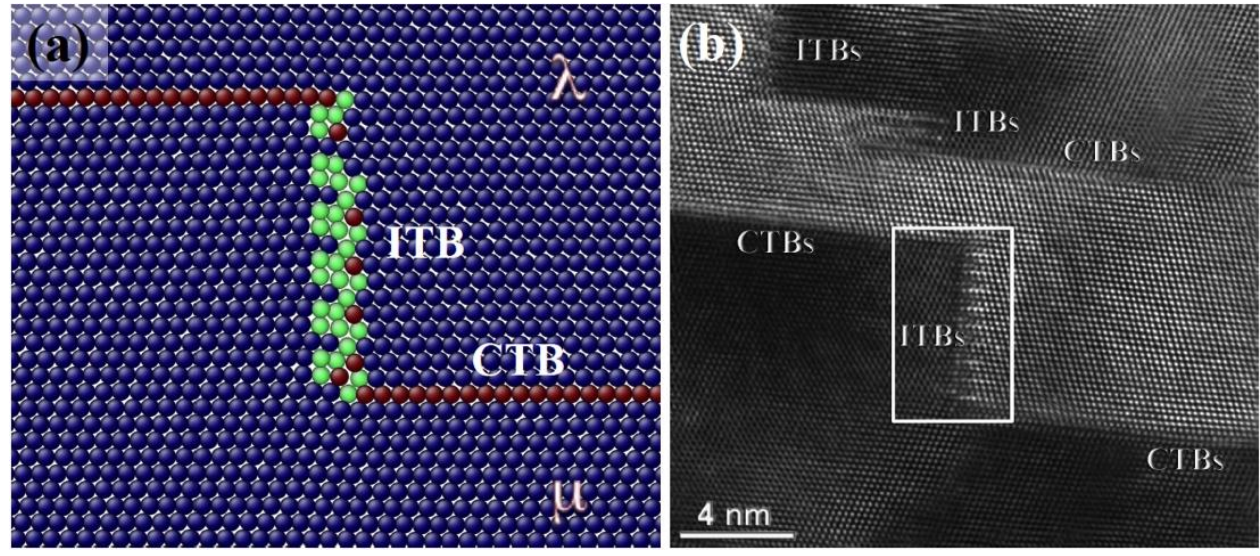

Figure 29. Incoherent $\{112\}$ facets on a $\Sigma 3$ coherent twin boundary in $f c c \mathrm{Cu}$ [112]. Atoms are coloured according to common-neighbour analysis.

The riser of a unit disconnection could be considered to have disclination character. However, the entire array is in an atomistic range where smearing the discrete dislocation is unphysical. It is better just to assign a net $b$ to the unit disconnection. If the step were slanted the effects in Figure 22 would be described as a spacing defect, which has a weak elastic field [86].

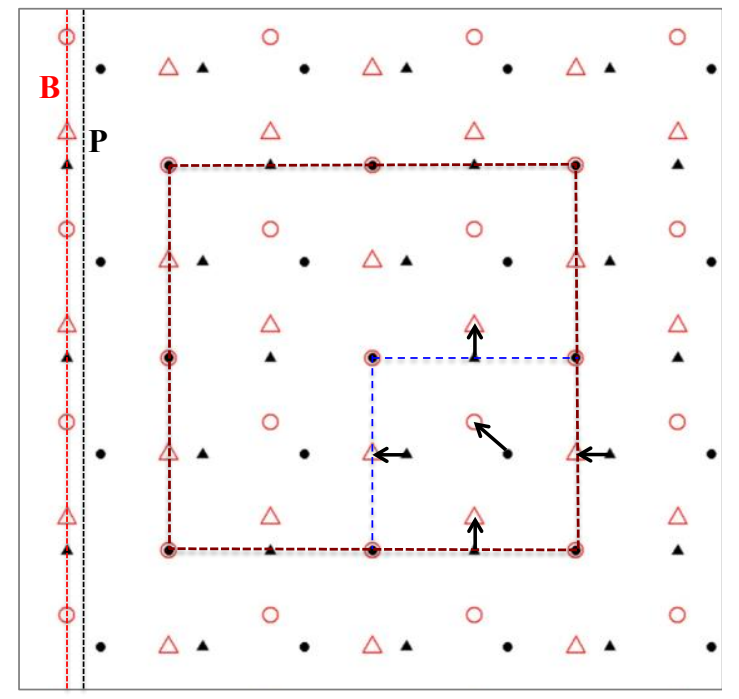

Figure 30. Nucleus with interfaces in $\beta$ structure. Pure shuffle vectors that create the nucleus are shown. 


\section{Twin nucleation}

\subsection{Homogeneous nucleation}

Figure 30 shows a doubly coherent $\mathrm{CDC}$ for $h c p$ with $\mathrm{P} / \mathrm{B}, \boldsymbol{\beta}$ structure into atomic coincidence. The configuration indicates a possible homogeneous nucleus for a twin. The CDC shows the general shuffle vectors for the creation of a $\lambda$ twin in a (black) $\mu$ matrix. Following standard nucleation theory, the nucleus is expected to be coherent $[127,128]$. This is also reflected by the critical thickness concept which states that below a critical size in the nm regime, misfit dislocations are unstable [48, 129-131]. The top and bottom interfaces are $\mathrm{B} / \mathrm{P}$ and the lateral interfaces are $\mathrm{P} / \mathrm{B}$ in the $\mu \rightarrow \lambda$ sense.

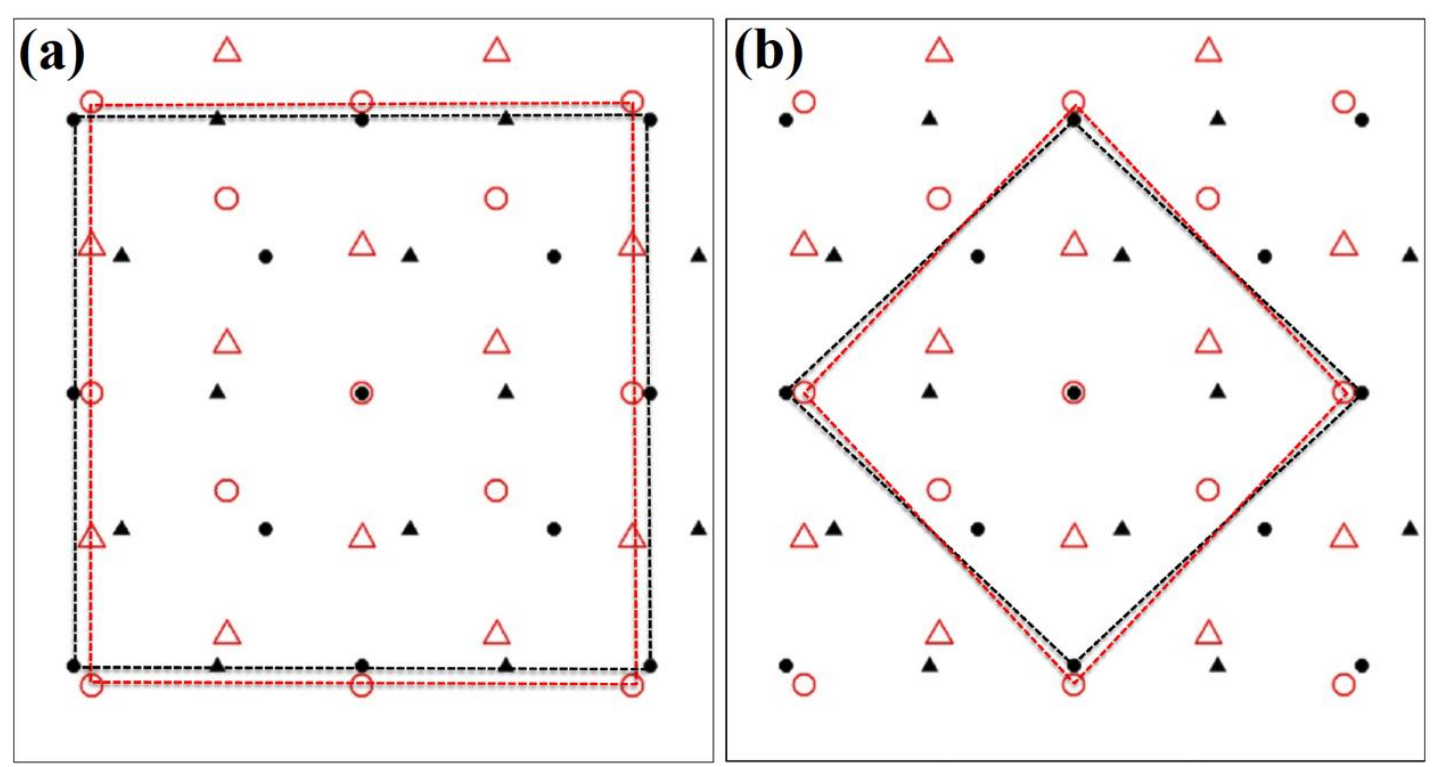

Figure 31. Two illustrations of the strains needed to create the nucleus. (a) Compression $\varepsilon_{z z}$ and extension $\varepsilon_{y y}$. (b) Shear $\varepsilon_{y z}$ in coordinates rotated be $\pi / 4$.

Figure 31 illustrates two ways of depicting the elastic displacements to create the nucleus from an unstrained matrix. The misfit strains, confined to the nucleus by the compliance of the matrix, are compressive in the $\mathrm{z}$-direction and tensile in the y-direction as shown in Figure 31(a). Interestingly, there would be no mismatch if the c/a ratio were ideal. The displacements can also be viewed as a shear diagram as in Figure 31(b). The latter clearly shows that the rotations at the corners (facet junctions) are in opposite senses. There are large stresses associated with this incompatibility. There will also be 
smaller strains associated with the differences in $\boldsymbol{p}$ between the interfaces, Eq. (9), because the actual structure is $\beta$, shifted from $\alpha$ by $\boldsymbol{p}$, Therefore, there would be junction dislocations at either end of the steps with equal and opposite Burger vectors given by Eq. (9).

The growth of such a nucleus, as simulated for $\mathrm{Mg}$, showed that the nucleus grew by $h(2)$ disconnection pair nucleation and lateral propagation, Figure 32(a). The nucleation of a disconnection pair is analogous the nucleation of a kink-pair on a dislocation traversing a Peierls barrier. Details of kink processes are beyond the scope of this work. Above a size of $3 \mathrm{~nm}$, TDs accumulate at corners and convert to $45^{\circ},(10 \overline{1} 2)$ facets, Figure 32(b). These still have junction defects at corners, the same as in Figure 27. Above $4 \mathrm{~nm}$, stress relieving disconnections and misfit dislocations form, and remain at the interface stresses become minimal, and the twin assumes a less regular shape, Figure 32(c).

There is a special category of homogeneous nucleation entailing coincidental overlapping of wide stacking fault ribbons in low stacking-fault-energy materials. This has been observed in nanocrystals $[132,133]$.
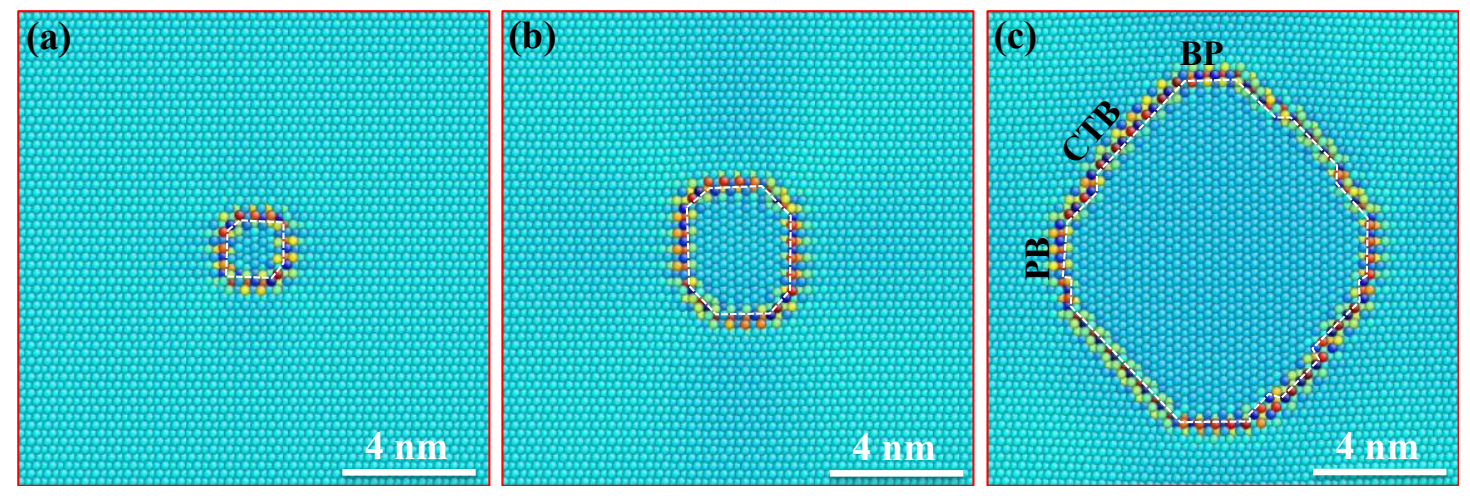

Figure 32. MD simulations of successive stages of growth of an embryo in Mg. Atoms are coloured according to their excess energy.

\subsection{Heterogeneous nucleation}

Heterogeneous nucleation, with emphasis on nanocrystals, is reviewed in [34]. There have been a number of observations of heterogeneous nucleation of TDs from 
grain boundaries, e.g., [34, 39, 134, 135]. There are several variations of heterogeneous nucleation at spiral dislocations, summarized in [62]. In the present context, the resulting twins resemble that in Figure D-1. In the standard manner, heterogeneous nucleation is more likely than homogeneous nucleation. Relevant to the present work, TEM observations of (10 $\overline{1} 2)$ twins in $\mathrm{Cu}, \mathrm{Al}$ and Ni nanocrystals nucleated at a grain boundary [136] revealed that the twins had no, or at most a very small, net Burgers vector. In this simpler case there are no misfit stresses. Nucleation of a single TD, say $\mathbf{\delta A}(\mathrm{d})$ leaves an opposite sign dislocation superposed on the boundary, with its accompanying stress field. This would favor the nucleation of a different partial, say $\delta \mathbf{B}(\mathrm{d})$, on the next plane to reduce the attendant strain energy relative to $\boldsymbol{\delta} \mathbf{A}$. Continued nucleation, including $\mathbf{C} \boldsymbol{\delta}$, of all three TDs in nominally equal numbers, would leave a nearly stress free twin boundary. Dynamic HRTEM studies of $\mathrm{Cu}$ [97] revealed a similar mechanism, where impingement of a lattice dislocation on a boundary led to a repeated nucleation source for TDs.

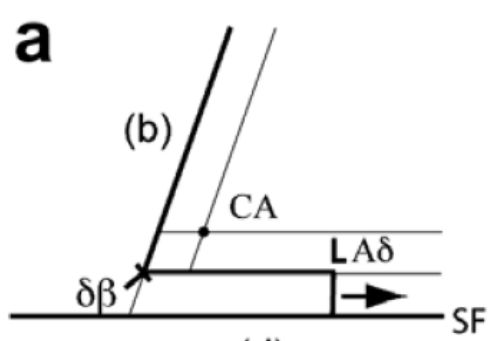

(d)

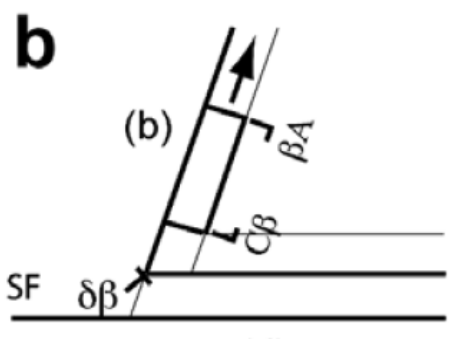

(d)

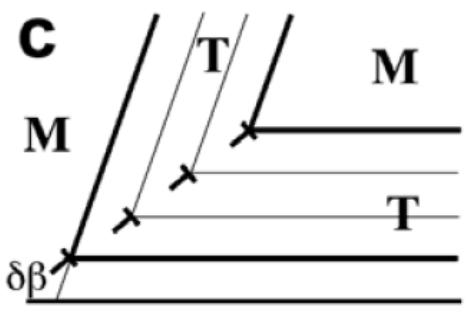

M

Figure 33. Schematics of the nucleation and growth of a V-shaped double deformation twin [137]. (a) Nucleation of a twin on the (d) plane. (b) Nucleation of the second twin on the (b) plane. (c) A V-shaped double twin after some growth. A stair-rod dislocation $\delta \beta$ is at every intersection between the (d) and (b) planes.

Another heterogeneous mechanism is nucleation arising from the impingement of a partial dislocation $\mathbf{C} \boldsymbol{\beta}$ onto a pre-existing stacking fault or twin boundary [137]. The partial splits into a stair rod dislocation $\delta \beta$, a TD with Burgers vector $\mathbf{A} \delta$, and the crossslipped dislocation $\mathbf{C A}$, which dissociates into $\mathbf{C} \boldsymbol{\beta}$ plus $\boldsymbol{\beta A}$, Figure 33. This would not occur without a stress providing a Peach-Koehler force. As seen in Figure 33, the 
process can then continue creating a $\Sigma 3$ twin on both the (b) and (d) planes, in a so-called "V" configuration. A tilt wall of $\boldsymbol{\delta} \boldsymbol{\beta}$ stair-rods is left at the intersection of the two twins. This tilt wall is the physical manifestation of a $\Sigma 9$ twin boundary. A similar mechanism leads to the formation of " $\mathrm{T}$ " and " $\mathrm{X}$ " intersecting twins. All of these have been observed in TEM [137].

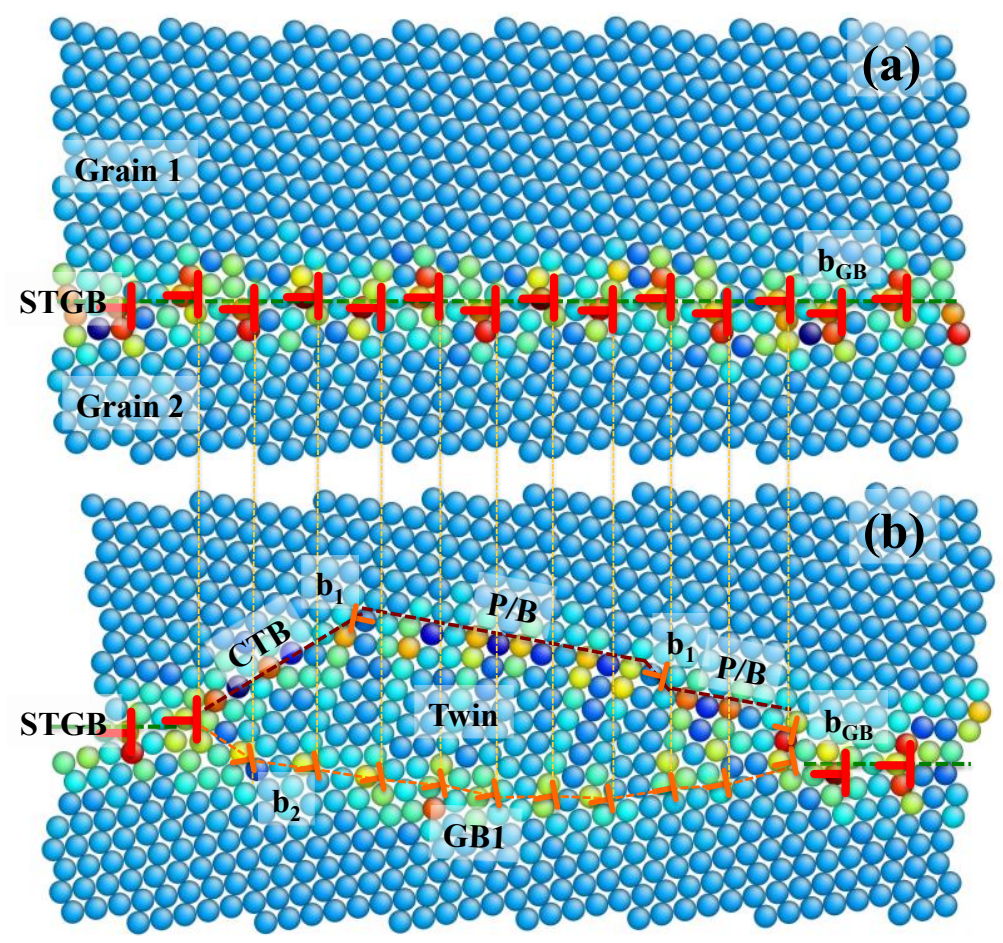

Figure 34. Atomic structures correspond to twin nucleation at a symmetric tilt boundary (STGB) in Mg [138]. Before twinning, grain boundary dislocations, $\boldsymbol{b}_{\boldsymbol{G} \boldsymbol{B}}$, are uniformly distributed along the STGB with an average spacing of $0.7 \mathrm{~nm}$ (including three atomic planes), and the Burgers vector is equal to $(0,0.256,0) \mathrm{nm}$. After twinning, a twin nucleus is outlined by the new boundaries (GB1), P/B and (1012) coherent twin boundary (CTB), respectively.

A twin nucleus with a different feature was found for a simulated (1012) twin in Mg [100, 138]. Under a shear stress the critical-sized nucleus had $8 h(2)$ TDs and one opposite sign [1011] dislocation with $\boldsymbol{b}=-0.4675[\overline{1} 011]$ for $\mathrm{Mg}$. The latter formed to compensate an emissary dislocation $\boldsymbol{b}=0.4675[10 \overline{1} 1]$ that formed and retreated through the twin embryo. As a result, the net Burgers vector of the emitted disconnection was 
halved. The emissary motion is opposite to that in the usual concept for emissary dislocations $[95,96]$.

These heterogeneous nucleation models entailed the motion of TDs with a dislocation component. A heterogeneous nucleation mechanism analogous to that in Figure 34 was observed in a MD study of $\mathrm{Mg}$ [39, 138-140]. Mechanisms of this type have been postulated [128] but had not been observed prior to that in [138]. The observed nucleation involved a pure shuffle mechanism with the same shuffle vectors as in Figure 30.

(a)
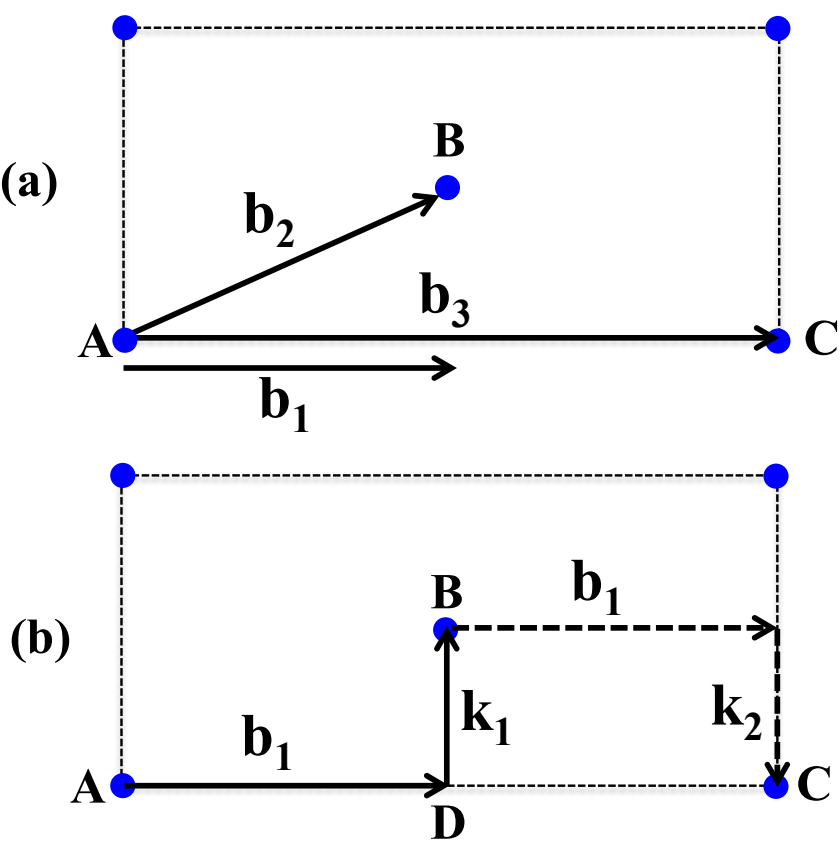

Figure 35. (a) A (100) plane in a bct crystal. Perfect Burgers vectors $\boldsymbol{b}_{2}$ and $\boldsymbol{b}_{3}$ and the dislocation component $\boldsymbol{b}_{1}$ of a Kronberg defect are shown. (b) Kronberg vectors $\boldsymbol{k}_{1}$ and $\boldsymbol{k}_{2}$ are depicted.

\section{Synchroshear}

Kronberg [141] proposed a synchroshear model as a possible low-activation energy process for slip and twinning in alumina. A simple example is shown in Figure 35 for a body-centered tetragonal crystal. One imagines a dislocation line present at A. For the ordinary perfect dislocations, the sheared by $\boldsymbol{b}$ to positions $\mathrm{B}$ or $\mathrm{C}$ and the process continues. For the synchroshear case in the first stage, the Burgers vector is an edge $\boldsymbol{b}_{k}$, but the extra plane is sheared to position D. In the second stage, the Burgers vector is 
again $\boldsymbol{b}_{\boldsymbol{k}}$, and the extra plane is sheared to position C. We call the shifts in the y-direction Kronberg vectors $\boldsymbol{k}$. The synchroshear defects are perfect, but are accompanied by alternating shifts by the $\boldsymbol{k}$ vectors. The pair of \pm shifts is somewhat analogous to shuffles, but in synchroshear entire planes shift rather than rows of atoms in the shuffles considered here. One could call these defects veers, since the defect displacements veer from being linear. At an initially planar free surface normal to $y$, the surface would become rumpled on one side of the glide plane after the defect had passed. The motion of the synchroshear is more complex than a simple dislocation and probably would require a larger activation energy. However, because the Burgers vector is smaller than those for the perfect dislocations, its elastic energy (line tension) would be less. Synchroshear is a candidate process whenever the perfect dislocation Burgers vectors are large as in this example.

Another example is prism glide in an $h c p$ crystal. A perfect dislocation with Burgers vector $\boldsymbol{b}=[0001]$ on a $(10 \overline{1} 0)$ plane has a large Burgers vector with a corresponding large elastic energy. The dislocation can be replaced by a perfect synchroshear defect with half the Burgers vector and Kronberg shift character, see Fig. 11-11 in [62]. In this case the $\boldsymbol{k}$ vector has a component normal to the glide plane, and the defect would be imperfect and have an associated stacking fault since the Burgers vector equivalent to $\boldsymbol{b}_{1}$ in Figure 36 would shift atoms from A to B basis sets or vice versa. In this case, a second imperfect defect with the same $\boldsymbol{b}_{1}$ would be needed to remove the stacking fault.

Twinning in Laves phases [142, 143], where synchroshear is described in detail, again entails large Burgers vectors. There is an analog for $h c p$ crystals [121]. We refer to the original papers for details in this more complex case. Synchroshear is a possibility For TDs on P/B planes as mentioned in Sect. 4.5. A more complicated possibility is shown as the displacement vectors in Figure 16(b). These displacements have $\boldsymbol{b}, \boldsymbol{s}$, and $\boldsymbol{k}$ components. A simulation where synchroshear is observed is for the glide of an interface dislocation on the incoherent $\{112\}$ plane for a $\Sigma 3$ twin in $f c c$ [144]. 

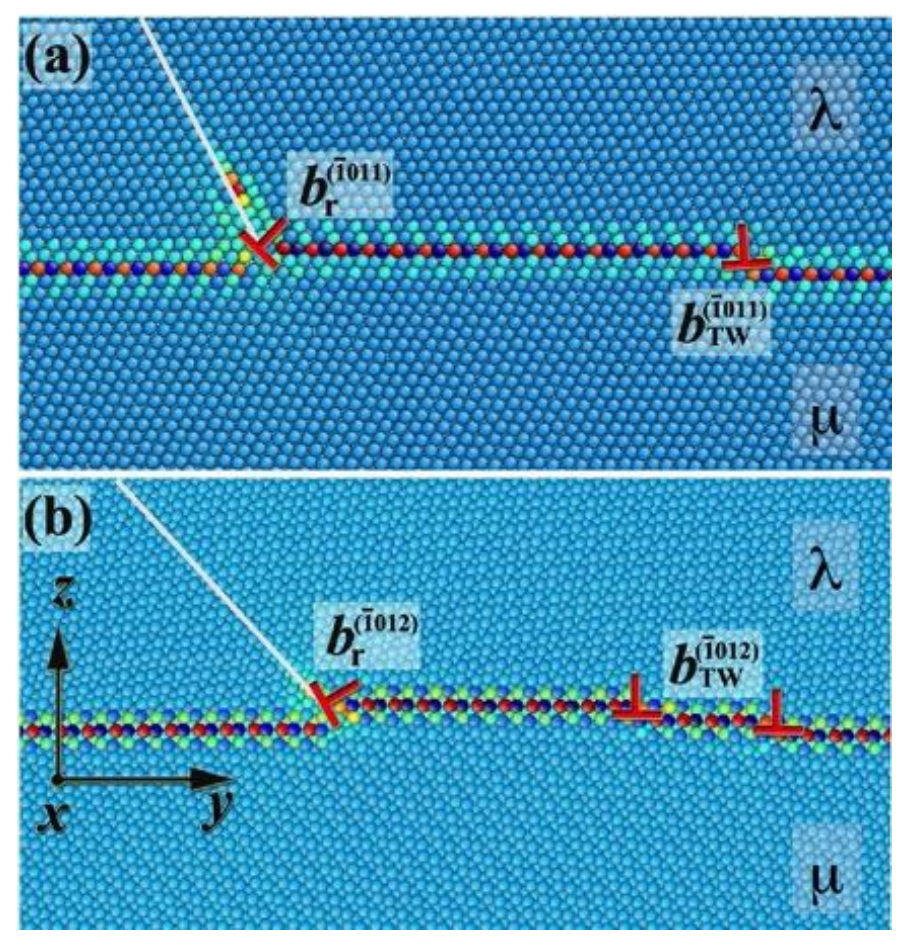

Figure 36. Lattice dislocations $a$ on a (0001) plane impinging on twin boundaries and reacting to form TDs and a residual disconnection $\boldsymbol{b}_{\mathrm{r}}$ [39]. Atoms are coloured according to their excess energy.

\section{Interaction of matrix dislocation and twin terrace}

The concept that the impingement of a lattice dislocation on a stacking fault or twin boundary can lead to local dissociation reactions and the creation of gbds or TDs is well known [145-148] and has received recent attention [39, 149, 150]. Results from the latter work are shown in Figure 36. An impinging 1/3[2 $\overline{1} \overline{1} 0]$ (0001) lattice dislocation reacts at the twin boundary to form one or two TDs with approximate Burgers vectors $\boldsymbol{b} \cong 1$ / 15 [10 $\overline{1} 1]$ and a sessile residual dislocation, $\boldsymbol{b}_{\mathrm{r}}$. For case (b) the impinging dislocation has a larger $b_{\mathrm{y}}$ component, sufficient to form two TDs [39]. This process has also been observed in HRTEM in $\mathrm{Cu}$ [97]. Continued intersections [149] or cross slip of the sessile dislocation [97] lead to propagation of the twin.

Twins can form to accommodate stress concentrations at pileups, disclinations, or as secondary twins to relieve stresses at a twin tip. An example of the former [150] is shown in Figure 37. 


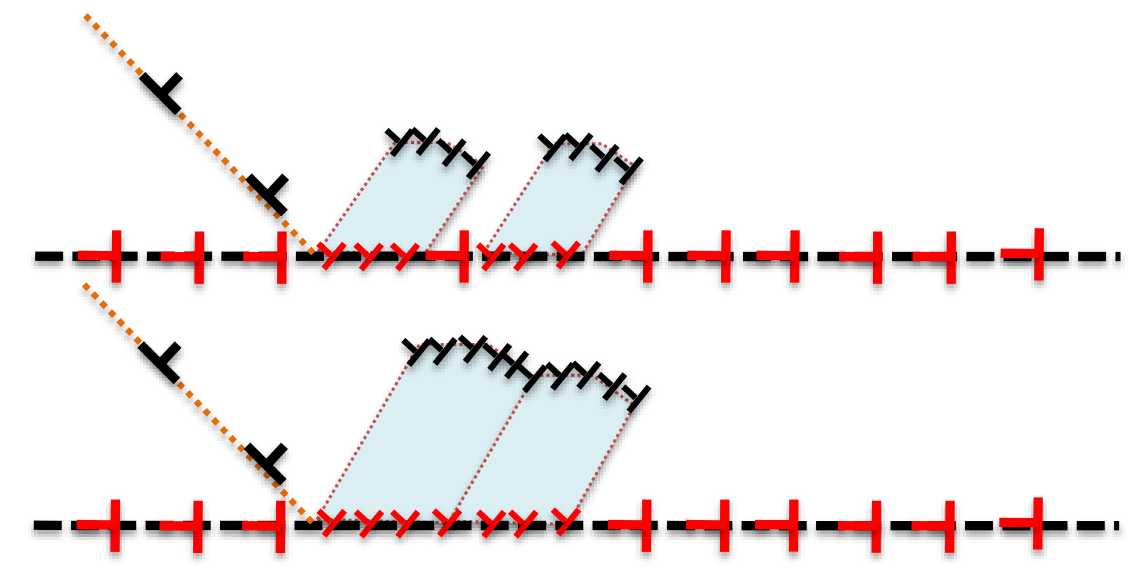

Figure 37. Accommodation twins formed in $h c p \mathrm{Mg}$ [150]. Here a dislocations pileup on a (0001) glide plane at a grain boundary barrier, leading to twin formation.

\section{Incoherent boundaries and the plane of shear}

There are two types of boundary other than the commensurate $K_{1}$-type twin plane that are of interest. We first consider "incoherent" boundaries: $I$ nominally normal to the Burgers vector $\boldsymbol{b}$ of the TD and $S$ in the plane of shear $S$ normal to both $K_{1}$ and $\boldsymbol{b}$. Because diffraction effects differ when viewing the $S$ plane in TEM, it has been called the "dark-side" plane [151]. That is, when the twin boundaries are relaxed as shown in Figure 38, a beam aligned with $x$ or $y$ would produce twin diffraction spots but no spreading of the spots since no long-range strains are present. The $I$ face viewed in HRTEM revels the offsets associated with $\boldsymbol{b}$. However, since $\boldsymbol{b}$ is parallel to y, the offset displacements are not visible when for a beam parallel to $y$.

The stressed configuration of Figure D-1 is relaxed by accommodation slip (or secondary twinning). Simple accommodation, often observed [32, 34], entails one emissary slip system [95, 96]. For a twin spanning a thin crystal, the twin interface can be accommodated instead by rotation (see Figure 47), but incompatibility constraints prevent this in the usual situation. Figure 38 illustrates the emission process for a (111) $f c c$ twin with $\mathbf{A} \boldsymbol{\delta}$ TDs. Traditionally, the dislocations emanate into the matrix. As in Figure 38(c), loops of edge lattice dislocations $\mathbf{A B}$ and $\mathbf{A C}$, each on every third plane, are emitted, leaving opposite sign dislocations in the boundary. These dislocations react as 
$\mathbf{A C}+\mathbf{A} \boldsymbol{\delta}=\mathbf{C} \boldsymbol{\delta}$, another TD variant. The result is a stress-free $\{112\}$ twin boundary, Figure 38(c). The emitted dislocations wrap around to the $S$ plane and create a stress free $\{110\}$ screw wall, Figure 38(e). Alternatively, the dislocations can emit into the twin, Figure 38(d) [146]. This should be a more likely process, since it also creates a stress free arrangement at the source. Another possibility, observed in [152] is that loops of lattice dislocations $\mathbf{A B}$ and $\mathbf{A C}$ are emitted on the $S$ plane, and wrap around to create the incoherent boundary in Figure 38(b). More generally several slip systems can become operative and up to four slip systems may be needed [153] to achieve accommodation.

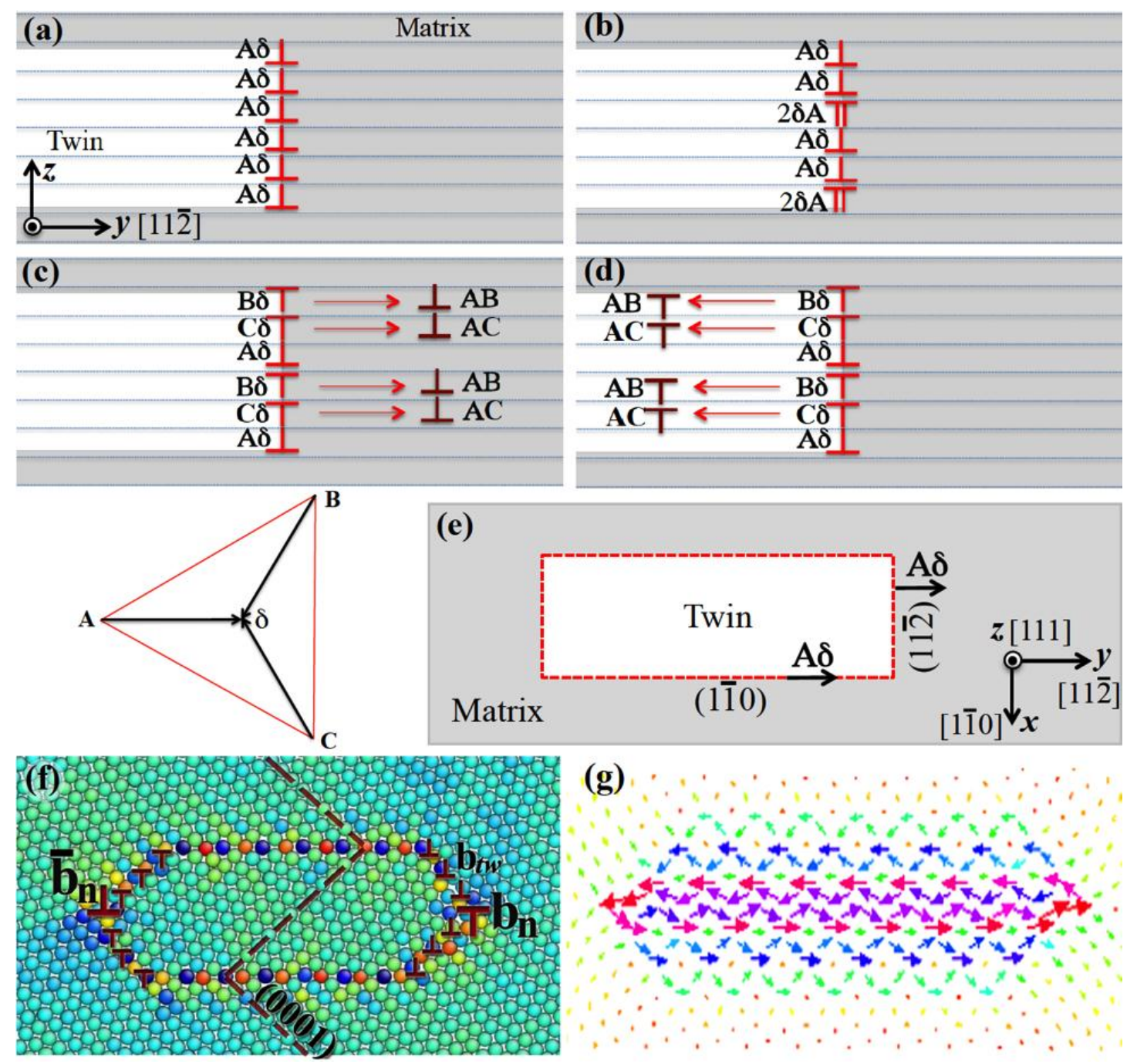

Figure 38. (a) Incoherent twin boundary $I$ in $f c c$. (b) A second possible $\{112\} I$ structure. (c) Resultant stress-free incoherent $\{112\}$ twin boundary formed by emission of lattice dislocations into the matrix shown, and (d) alternative in forming (c) by emission of lattice 
dislocations into the twin. (e) Top-view of a twin domain. The red dashed line indicates typical TD loops. The TD is edge type on the [112] I plane and screw type on the [11̄0] $S$ plane. (f) $I$ planes of a near zero shear (1012) twin in hcp Mg, containing 6 twinning dislocations that has Burgers vector of $0.05 \mathrm{~nm}$ and one partial dislocation that has Burgers vector of $-0.35 \mathrm{~nm}$. Atoms are coloured according to their excess energy [139]. (g) Displacements of atoms in the twin domain in (f).

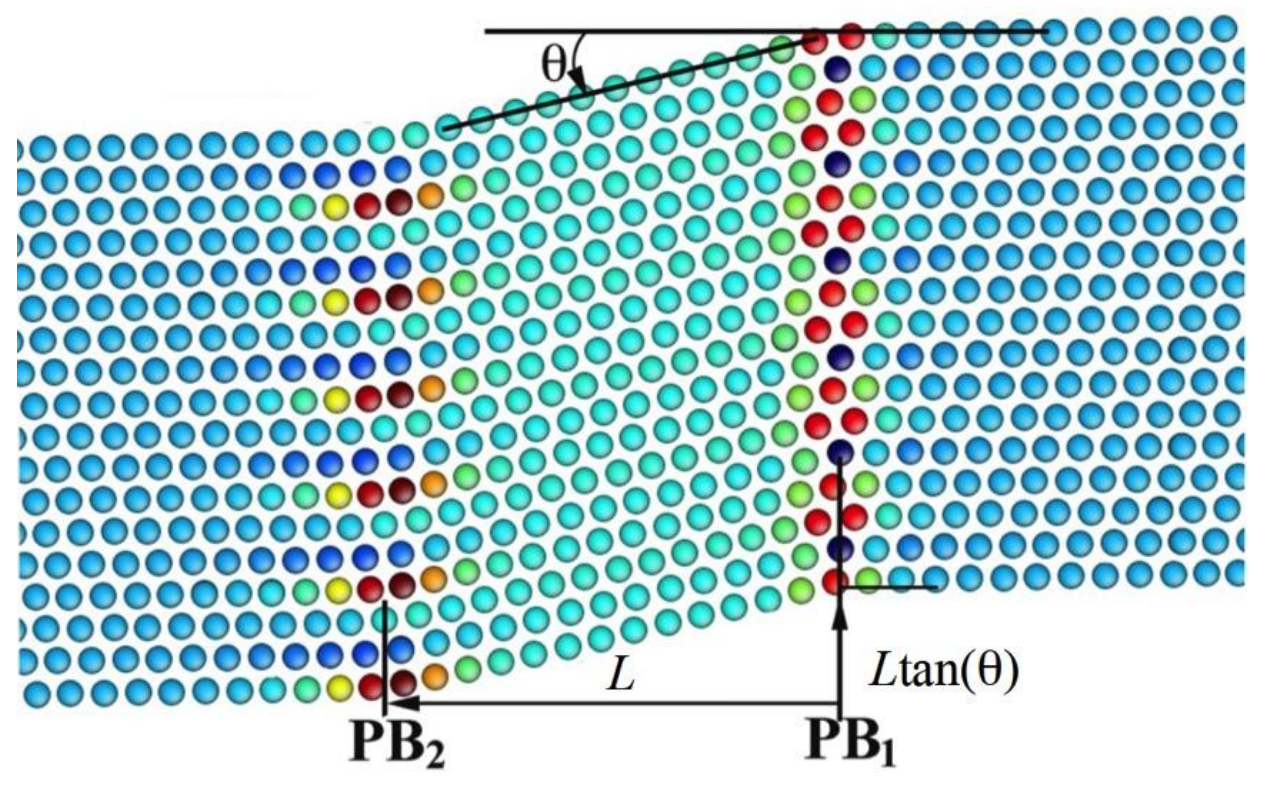

Figure 39. Boundary in Fig. 38c dissociated under stress to create a region of 9R structure that is bounded by two new boundaries: PB1 and PB2. The stacking sequence is $\boldsymbol{B C B C A C A B A}$. Atoms are coloured according to their excess energy [144].

Simulations and HRTEM reveal that the structure in Figure 38(c) is stable, but that the possible structure in Figure 38(b) is metastable [154] and, consistent with earlier simulation results [155]. A simulation of a $\{112\}$ twin boundary in $\mathrm{Cu}$ [153] showed that the boundary, Figure 38(c), dissociated by $\sim 1 \mathrm{~nm}$ into a 9R structure, in agreement with earlier work [156]. A MD simulation for $\mathrm{Cu}$ [144] showed that the dissociation increases under an applied stress to thicknesses of $\sim 5 \mathrm{~nm}$, Figure 39, creating a bulk region of the $9 \mathrm{R}$ structure. This is of interest as an example of a bulk phase transformation stable only under stress. Subsequent HRTEM studies verified this vestigial dissociation [155-160]. 
Another possibility, analyzed in [161] is that the $S$ plane in $f c c$ reconstructs into two $\{112\}$ planes, so that a view along [111] in an idealized case would have a hexagonal cross-section with all three $\{112\}$ planes with the same [1 $\overline{1} 0]$ zone axis present. Simulations of the Gibbs boundary energy as a function of rotation about a $\langle 111\rangle$ zone axis showed that the reconstruction was energetically favorable in accord with the GibbsWulff plot in Figure 40. In the idealized case one then would expect a wrap-around array of mixed dislocations on the two reconstructed $S$ planes.

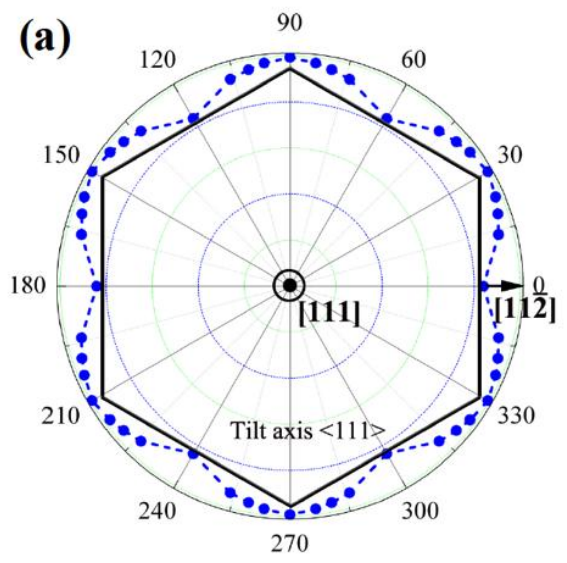

(b)

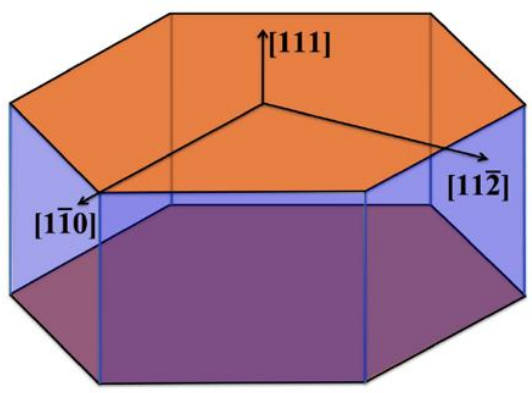

Figure 40. Gibbs-Wulff plot predicting the equilibrium shape in (b) [161].

For a long, thin $K_{1}$ type twin, dislocations emitted at the incoherent boundary are unlikely to wrap around. This leaves incompatibility on the $S$ plane equivalent to a twist disclination. This incompatibility can be removed by the emission of screw dislocations removing the elastic strain and producing a partitioned rotation [162]. The resultant structure in $f c c$ would be an orthogonal array of screws, one set left-handed and the other right handed $[162,163]$. In lower symmetry crystals, the screw network can relax to a mixed dislocation array to minimize line energy [62].

The configuration on the $S$ plane can differ markedly in other structures. An example is the $h c p$ twin where the $S$ plane was studied by HRTEM and by simulation [151]. For the $(0 \overline{1} 12)$ twin in hcp the relaxed $I$ boundary is that shown in Figure 38(f). The residual $\boldsymbol{b}$ created by the emission of a lattice dislocation [1 $\overline{100}$ ] is approximately six times larger than the TD Burgers vectors [100], and the repeat unit in the $\mathrm{z}$ direction is 7. This has the consequences depicted in Figure 41. The idealized twin in Figure 41(a) is 
viewed along the $-x$ direction, normal to the TD $\boldsymbol{b}$. The TDs on this $I$ plane would be edges with horizontal line directions and those on the $S$ plane, normal to $x$, would be screws with line directions toward the viewer. The actual structure is indicated in Figure 41(b). There the $S$ plane has developed facets with the $I$ plane structure. An HRTEM view of the $S$ plane is shown in Figure 41(c). The TDs are not visible because the Burgers vectors are so small and because they are so closely spaced. However, the large misfit dislocations are discernable as shown. The Burgers vectors agree with that of the large dislocation in Figure 38(f).

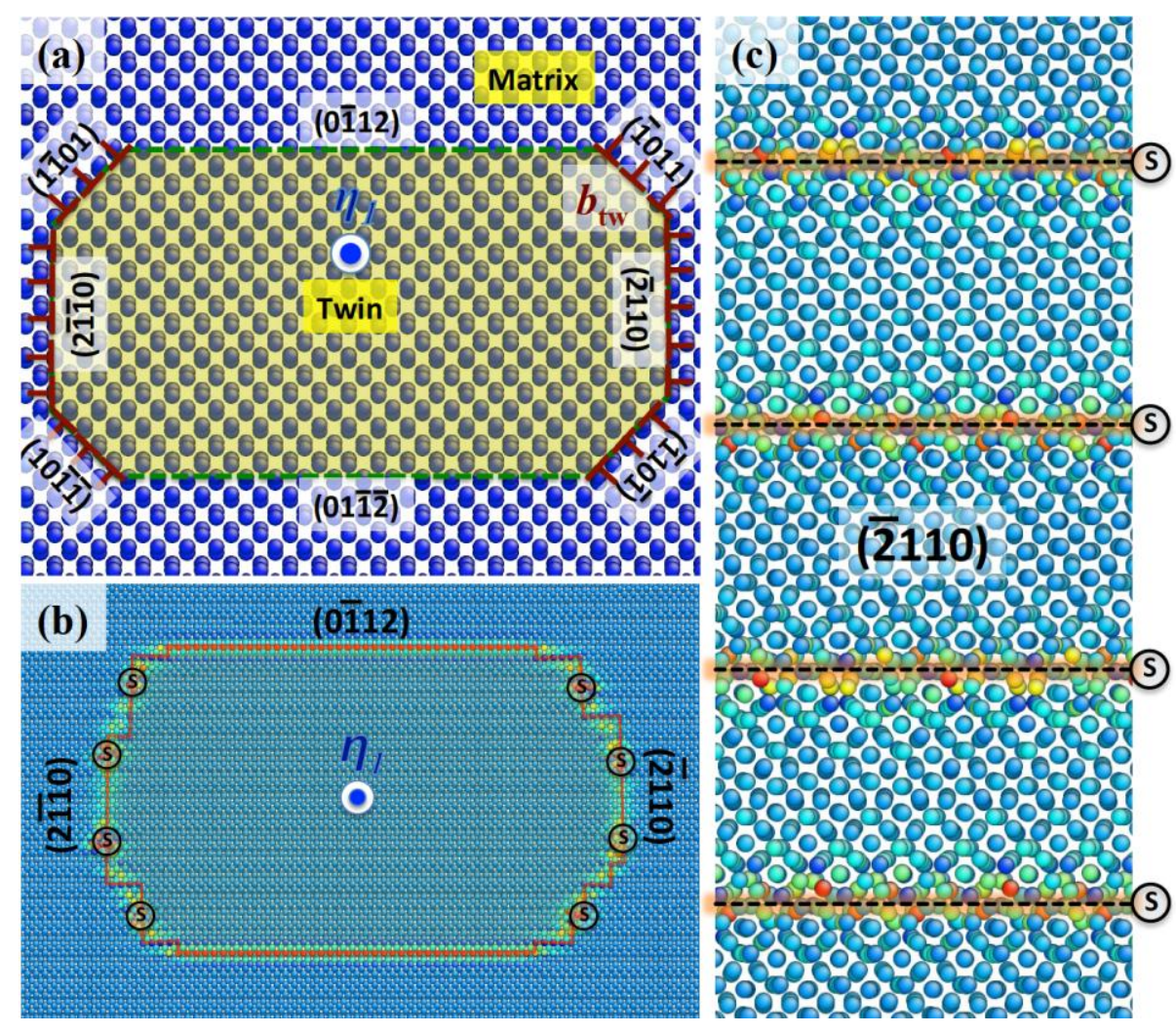

Figure 41. (a) An ideal twin structure viewed along the twinning shear direction [151], (b) The relaxed twin structure of the structure (a). (c) Plan-view of the (110) S plane, showing misfit screw dislocations. The patches between the large misfit screw dislocations contain the tiny, opposite sign, TD screws. Atoms are coloured according to their excess energy.

\section{Twin intersections and duplex twinning}


Twin intersections are a commonly observed topographical feature. The analysis of the defect configurations at such intersections is of interest in determining the stability of the intersections and their role in hardening and reversion. This Section presents results from our work using electron back-scattered imaging [164-165]. The first obvious example of twin-twin interaction is the accommodation of the stress field of a single lenticularly shaped deformation twin by a second twin forming near the tip where the stresses are largest. Observations include effects of twin growth, but the twins in $\mathrm{Mg}$ in Figure 42 indicate some correlation of this type $[164,165]$. The lack of penetration of a twin by a second twin, despite the stress-concentration/pileup nature of the tip of the impinging tip, shows that the first twin boundary is a strong barrier for the second twin. This effect is marked in $f c c$ crystals where the minimum angle between twin planes across the first twin is $39^{\circ}$, so the $\boldsymbol{b}_{\mathrm{g}}$ left in the interface after intersection is large. Also some crossings would require that the crossed TD is an $h(2)$ TD. Both factors make crossing more difficult. The strong barrier nature is reflected in Hall-Petch hardening in $\mathrm{Cu}$ [166], where $\{111\}$ twin boundaries were equally effective barriers as were grain boundaries.

Secondary twinning was also observed [165] as shown in Figure 43. An extreme example of accommodation by secondary twinning of this type is a uranium alloy where multiple twins, including multiple internal twins within a given twin, are observed [167]. These are analyzed as having twinning strains that roughly sum to the overall twinning strain. The five-fold twin in Figure 12 is another example. The twins depicted in Figure 37 formed by successive disconnection nucleation [150], but the same configuration can be formed by twin intersection, forming $\mathrm{V}, \mathrm{T}$, and $\mathrm{X}$-shaped twin intersections [149]. "Picture frame" boundaries are also possible [168]. Because if the high symmetry, the twin intersection boundaries and the domain line dislocation structures of the joint boundaries are simple: $\Sigma 9$ and $\alpha \delta$ or $\boldsymbol{\alpha} \delta / \mathbf{C D}$ stair-rods. For a higher order intersection in a deformed crystal, $\Sigma 27$ boundaries could be present. 


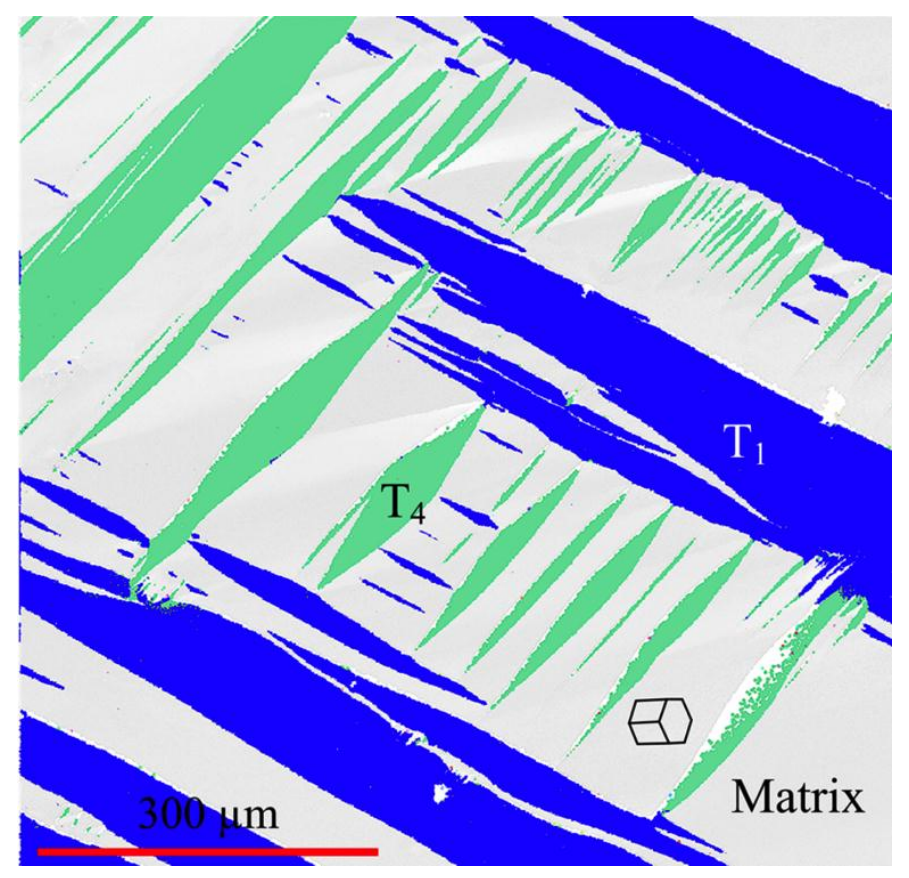

Figure 42. EBSD analysis shows quilted-looking twin-twin intersections observed in the specimen of Mg single crystal loaded along the [1010] direction [165].

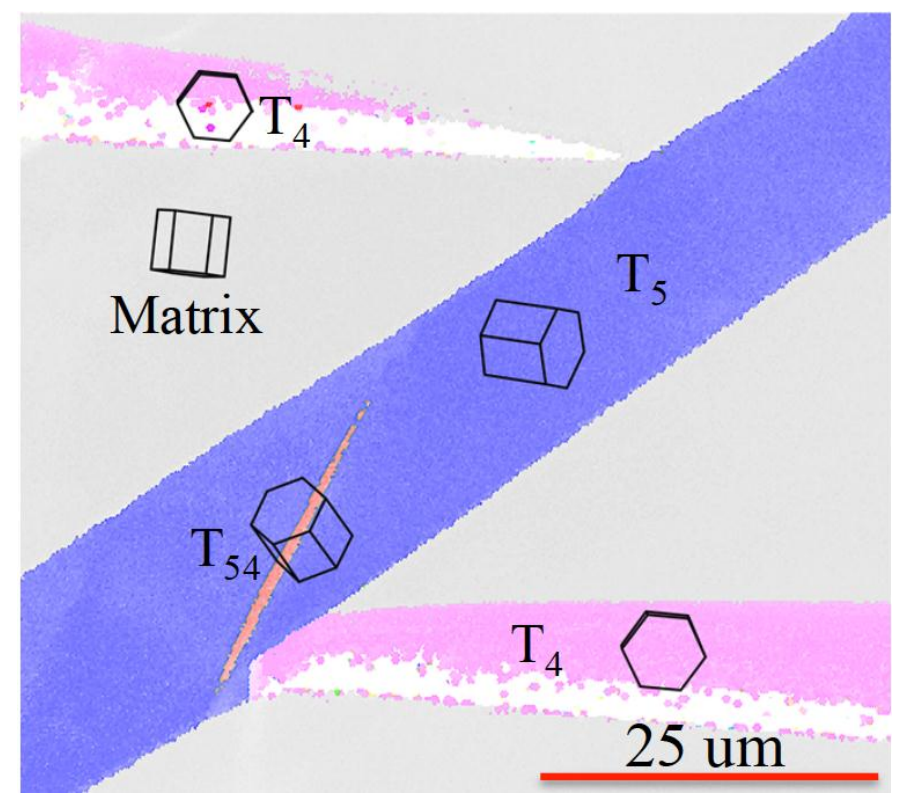

Figure 43. EBSD analysis shows double tension twin structures [165] in the specimen loaded along the [0001] direction in Mg. Secondary twin does not connect to an incoming twin, where a secondary twin $\mathrm{T}_{54}$ nucleated inside a primary twin $\mathrm{T}_{5}$. 
Recent simulations [164] and observations [165] of twin intersections have also been obtained for hcp Mg. The situation is not as simple as that in $f c c$ because of the lower symmetry and because a TD with a given Burgers vector is glissile in one direction but not in the reverse direction (the compression-tension asymmetry discussed in Sect 4.7). $V, T$ and $X$ intersection configurations were observed for intersections of $\{10 \overline{1} 2\}$ planes with the same zone axis. The Burgers vectors at the joint domain boundary are the sums of those for the TDs in the two twins but unlike $f c c$, they are irrational. Other intersections entail different combinations of $\{10 \overline{1} 2\}$ planes as shown in Figure 44. For the X configurations, which show "apparent" crossing", the twins do not actually cross: instead there is a region of differing orientation at the intersection. There are a number of possibilities that are discussed in [165], and for illustrative purposes we consider two of these.
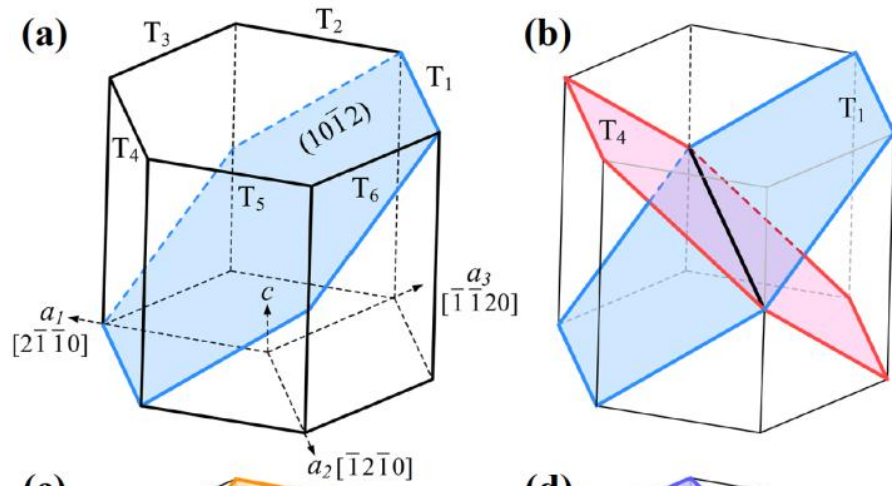

(c)

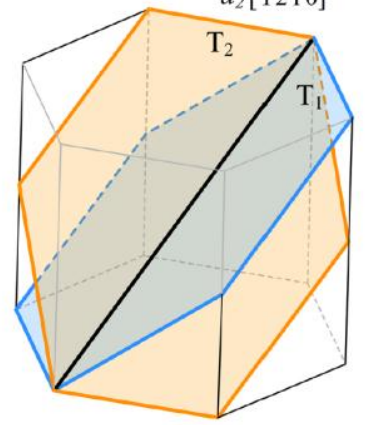

(d)

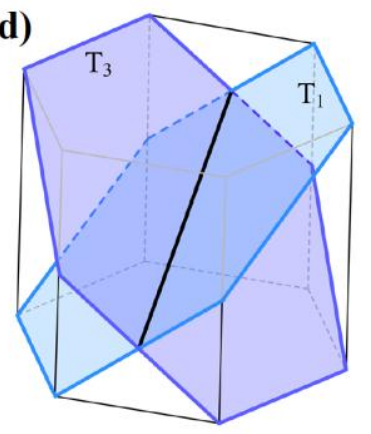

Figure 44. (a) The six $\{10 \overline{1} 2\}<\overline{1} 011>$ twin variants $\mathrm{T}_{\mathrm{i}}(\mathrm{i}=1$ to 6 ) in a hexagonal-closepacked $(h c p)$ structure can form three crystallographically different types of twin-twin interactions [165]; (b) Type I twin-twin pair $\mathrm{T}_{4} \leftrightarrow \mathrm{T}_{1}$ with the intersection line along [1리이, (c) Type II(a) twin-twin interaction $\mathrm{T}_{2} \leftrightarrow \mathrm{T}_{1}$ with the intersection along [ $\overline{2} \overline{2} 43$ ] and (d) Type II(b) twin-twin interaction $\mathrm{T}_{3} \leftrightarrow \mathrm{T}_{1}$ with the intersection line along [0 $\left.\overline{2} 21\right]$. 


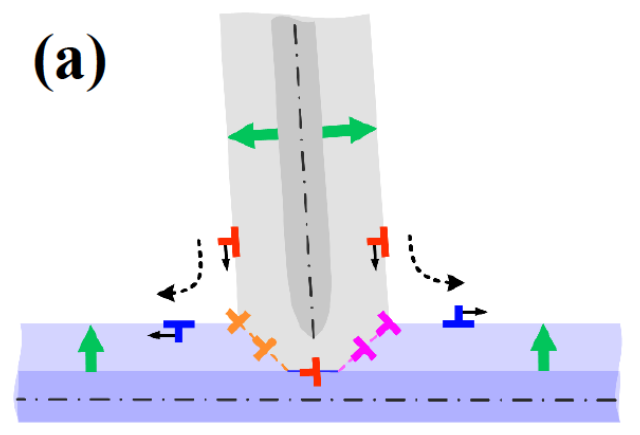

Dissociating
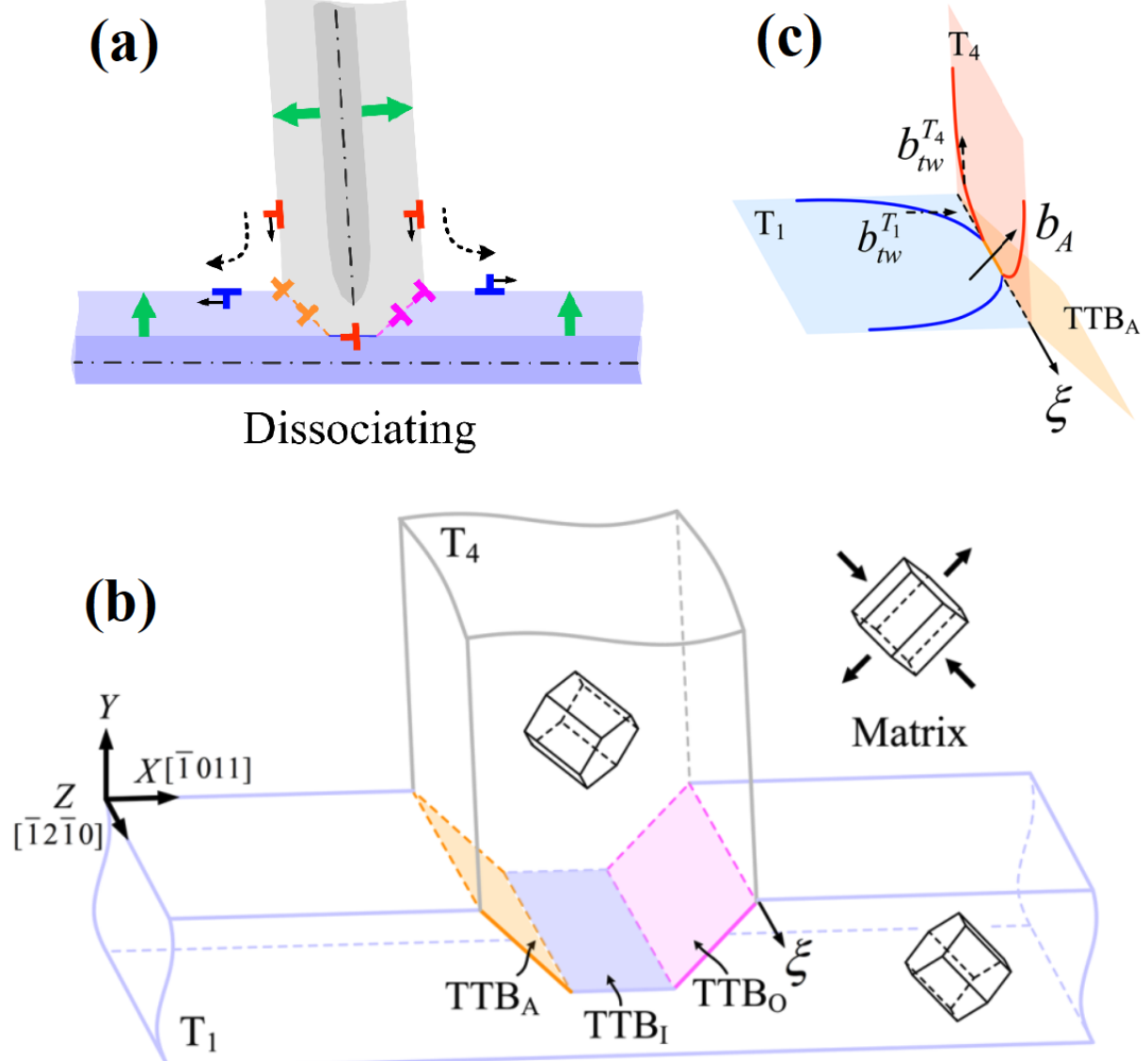

Figure 45. Formation mechanisms of twin-twin boundaries as a twin $\mathrm{T}_{\mathrm{i}}$ is approaching the boundary of a pre-existing twin $\mathrm{T}_{\mathrm{j}}(\mathrm{i} \neq \mathrm{j})$ [165]. (a) The dissociating mechanism where a TD dissociates into a TD associated with a second twin, leaving one junction. The light grey and light blue domains correspond to the $\mathrm{T}_{\mathrm{i}}$ and $\mathrm{T}_{\mathrm{j}}$ twins, respectively. The TDs are drawn in red $\left(\mathrm{T}_{\mathrm{i}}\right)$ and blue $\left(\mathrm{T}_{\mathrm{j}}\right)$ colours. Orange and pink colors are used for resultant junctions (or twintwin boundary dislocations) resulting from TDs reactions. (b) One twin-twin boundary is referred to as $\mathrm{TTB}_{\mathrm{A}}$ (acute angle), and the other is referred to as $\mathrm{TTB}_{0}$ (obtuse angle). (c) These boundaries are formed by the reactions of TDs.

Figure 45(a) shows a $\mathrm{T}$ configuration formed when two twins $\mathrm{T}_{1}$ and $\mathrm{T}_{4}$ with the same zone axis, type A, intersect. Figure 45(b) shows the analysis of the structure. The relative orientations are such that the joint intersection plane is near (0001) in each crystal. The actual angle between the (0001) planes is $7.4^{\circ}$. Hence, the domain line defects form a partitioned $7.4^{\circ}$ tilt wall. Once formed, the twins at the intersection can continue to grow by nucleation of TDs at the intersection or by impingement and reaction 
of a TD [165]. The tilt walls in the joint twin interface are equivalent to $7.4^{\circ}$ disclination dipoles. Hence they have a large elastic field and a large self-energy. They must relax on unloading by the formation of emissary accommodation dislocations in the twin or matrix. Reversion of the twins would be impeded by such a relaxation as well as by the energetically unfavorable requisite dissociation of the tilt dislocations. A second example is shown in Figure 46. The two twin systems do not share a zone axis, type B. Here the intersection of a $T_{1}$ and $T_{2}$ twin produces a variant of the $T_{2}$ twin rotated by $6.2^{\circ}$. In such examples the different joint intersection interfaces can have different tilt angles and the associated tilt dislocations can have screw components. For type A pairs, the original twin plane is a strong barrier as shown in Figure 42, analogous to the $f c c$ case. For type $\mathrm{B}$, the twin planes align within $7^{\circ}$ and hence are not as strong barriers $[169,170]$. Thus, while direct crossing of a twin by another twin does not occur, the shear is propagated through the first twin by successive TDs moving through the third twin present at the intersection.

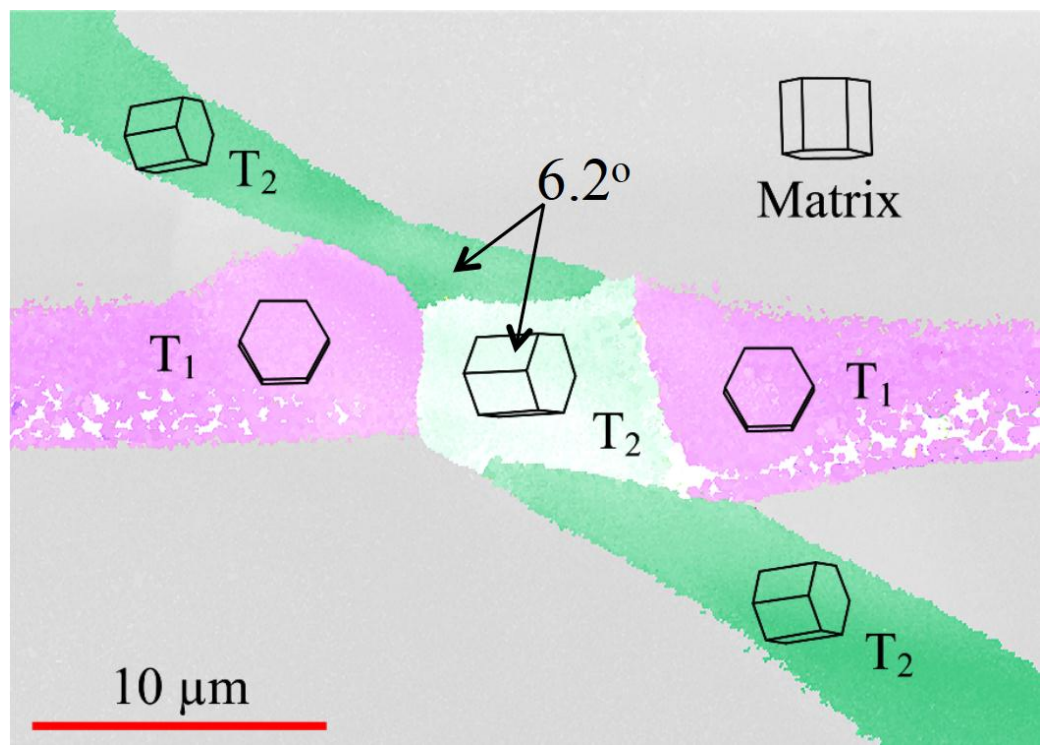

Figure 46. EBSD analysis shows 'Apparent crossing' twin structures [165] in the specimen loaded along the [0001] direction in $\mathrm{Mg}$, where two $\mathrm{T}_{1}$ twins and one $\mathrm{T}_{2}$ twin intersect. The intersected domain rotates about 6.2 degrees with respect to the original twin $T_{2}$. 


\section{Type II twinning}

If the system is unconstrained, the TD mechanism can form perfect type I twins as well as compound twins if a $K_{2}$ plane is available. The essential reason for this is that the engineering shear plane is parallel to the terrace twinning plane. For certain high symmetry cases, a compound twin with a perfect $K_{1}$ plane can also form by a "type II mechanism". That is, the twin is formed by TDs gliding on slant planes. This has been inferred in a number of descriptions, e.g., Fig. 23-3 in [62]. Here we provide a detailed proof that this is possible for (111) twinning in $f c c$. When the twins form by TD motion on $K_{2}$, the shear plane is inclined to the interface with consequences which we describe.

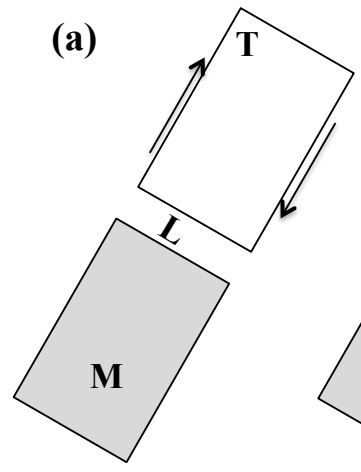

(e)

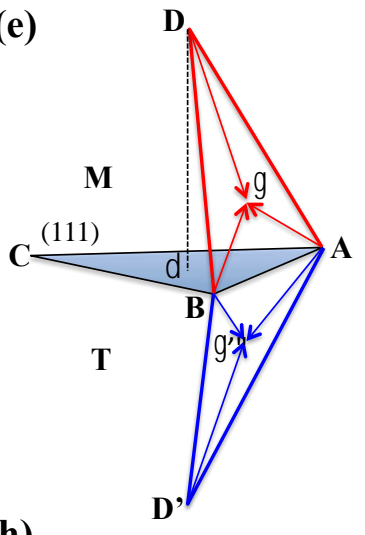

(h)

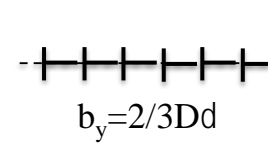

(b)

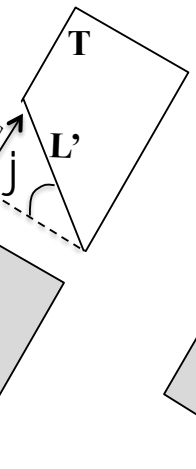

(f)

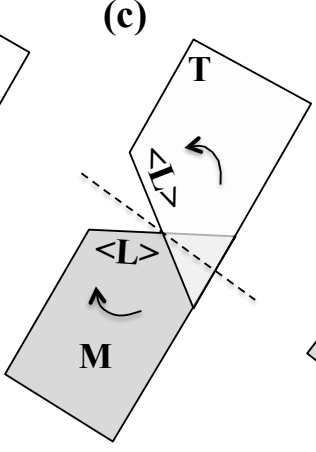

(d)

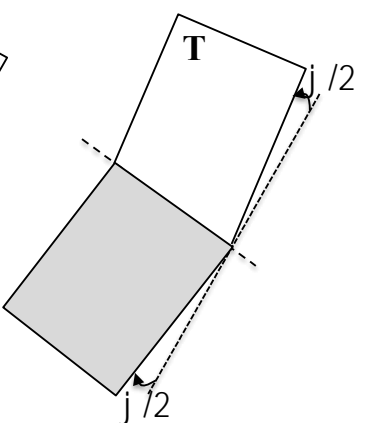

(g)
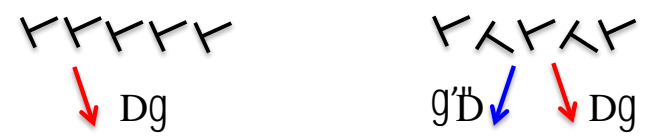

(i)

(j)

Figure 47. (a)-(d) Cut and distort operations to produce a twin. (e) Double Thompson tetrahedron. (f)-(h) Different representations of twin boundary. (i) Rotation angles producing interface (j). 
One can envision the creation of the twin as in Figure 47. The first step is to make a cut, Figure 47(a). The portion T, to become a twin, is then offset by an engineering shear strain $\gamma_{\mathrm{yz}}$, Figure 47(b). If the twin is simply rotated by $\varphi$, as in Figure 47(b) (the PTMC procedure), there is an incompatibility since $\mathrm{L} \neq \mathrm{L}$ '. The incompatibility is removed by the counter rotations $\mathbf{R}$ and $-\mathbf{R}$. This emphasizes the importance of partitioning the rotation. Analogous to the partitioning of strain at a coherent interface, any relative rotation between the adjoining crystals must also be partitioned, a major feature of the TM [171]. This is obvious if one considers a symmetric tilt boundary, formed by the same type of mechanism [52]. If the interface rearranges and partitions the rotations to $\varphi / 2$ in each crystal, the two lengths $\langle\mathrm{L}\rangle$ are the same, the twins bond without misfit and a twin is formed. In the anisotropic case or with differences in thickness of the two crystals, the partitioning is not equal [5].

The same result follows in a dislocation model. Figure 47(e) shows the double Thompson tetrahedron for this case and shows that the (111) type (c) and (c') planes are inclined at $2 \theta^{0}=140.64^{\circ}$ for a compound twin. Next, twinning dislocations, $\gamma \mathbf{D}$, glide on every (c) plane in the matrix, Figure 47(f). The rearrangement and rotation [5] creates the dislocation arrangement in Fig.46g, which could have been formed by alternating TDS $\boldsymbol{\gamma} \mathbf{D}^{\prime}$ and $\boldsymbol{\gamma}^{\prime} \mathbf{D}^{\prime}$ moving into the interface from opposite directions. The $\pm \mathbf{C} \boldsymbol{\delta}$ in-plane components cancel, creating a spacing defect [86] that extends the interface. The remaining normal vectors then form the tilt wall in Figure 47(g), with grain-boundarydislocation $(\mathrm{GBD})$ vectors $\boldsymbol{b}_{\mathrm{g}}=2 / 3 \mathbf{D} \boldsymbol{\delta}=2 / 9$ [1 $\left.\overline{1} \overline{1} \overline{1}\right]$. There are many forms of Frank's equation $[18,62]$, and for the present tilt wall, it is

$\varphi=2 \tan ^{-1}(b / 2 L)=2 \sin ^{-1}(b / 2 d)$

Here, $b$ is the magnitude of the TD Burgers vector, here $\gamma \mathbf{D}, \mathrm{d}$ is the interplanar spacing of $\{111\}$ planes and $L=d / \cos (\varphi / 2)$ is the spacing of GBDs in the interface. With these specific properties, the tilt wall of GBDs produces a tilt angle $\varphi / 2=70.32^{\circ}$. However, as shown in Figure 47, this is exactly the angle $\varphi^{0}$ between $\{111\}$ planes. Thus the twin, although formed by a "type II" mechanism is a compound twin. This result holds as well whenever the conjugate system $K_{2}$ is a variant of $K_{l}:$ e.g. (1012) and (1012) 
in $h c p$. The general condition for a compound twin are that either $\varphi=\theta^{0}$ as in the $f c c$ case, or, when $\varphi$ is relatively small, $\varphi=\varphi^{0}=90^{\circ}-\theta^{0}$ as in the $h c p$ case, Figure 48.

The procedure leading to Figure 47(f) is equivalent to that in the PTMC [25], when it is applied to twins [8]. There has been controversy about the relation of the TM and the PTMC [172, 173]. As stated a number of times [3, 7, 171], the PTMC is incomplete if there is a tilt component to interface dislocations because partitioning is not taken into account. If the PTMC angle $\theta^{\prime}$ is small, one can simply rotate the PTMC interface by $\theta^{\prime} / 2$ to obtain the equilibrium result: for small angles, the difference between $\theta^{\prime}$ and $\theta$ is negligible [7]. However, when $\theta^{\prime}$ is larger, the difference from $\theta$ is significant, $45^{\circ}$ versus $39^{\circ}$ in Figure 47. Hence, for larger angles, the full TM procedure must be used in partitioning.

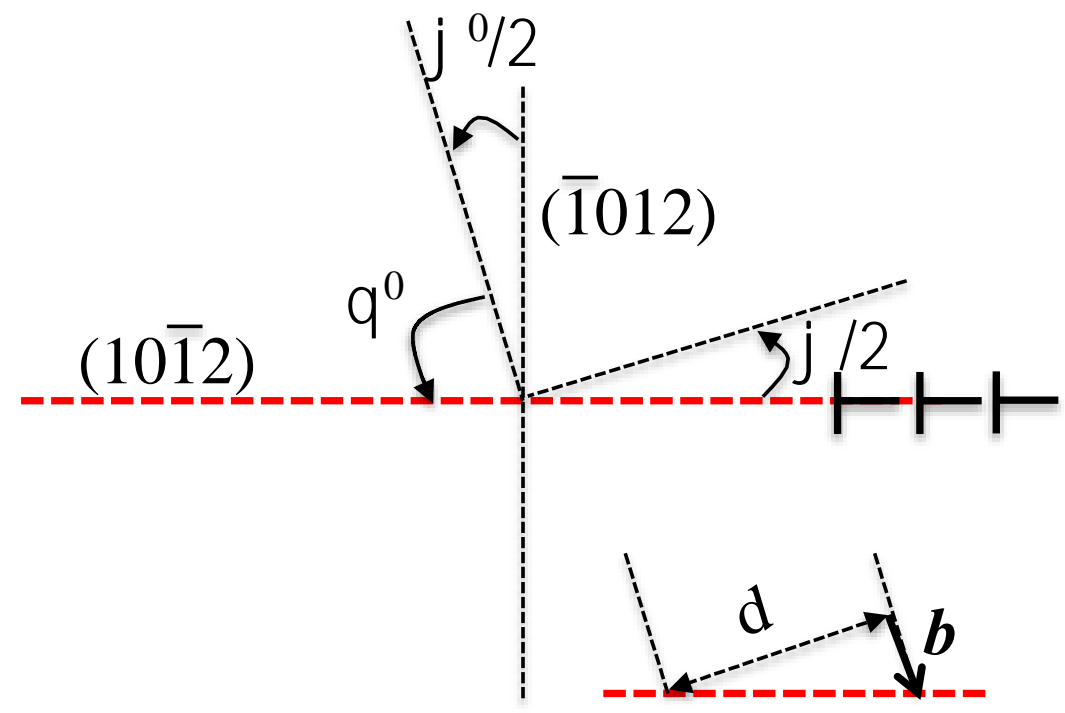

Figure 48. Rotation $\varphi$ produced by tilt wall of dislocations $\boldsymbol{b}$ with spacing $d$ and corresponding rotation of $(\overline{1} 012)$ plane.

There are other cases where the same physical model, TDs moving on a $K_{2}$ plane, does not produce a rational interface. This occurs when the conjugate $K_{2}$ plane, while having the same zone axis, is not a variant of the $K_{1}$ plane. An example is (10 $\left.\overline{1} 1\right)$ for $K_{1}$ and (1012) for $K_{2}$. The angle $\varphi$ for the type I mechanism is the same as for a $(10 \overline{1} 2)$

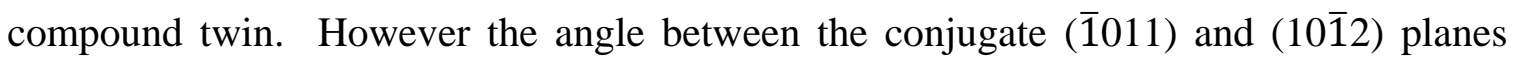


differs as shown in Table 2 for nine metals and for an ideal c/a. Hence, this conjugate system does not yield a compound twin. This is close to an extreme example of deviations between the two angles. The difference would be smaller for planes inclined at an angle close to $0^{\circ}$ or $90^{\circ}$. The classical statement for a compound twin is that all four twinning elements be rational. However, we see that an added proviso is: $K_{2}$ is a variant of $K_{1}$. Bevis and Crocker [108] have indicated that a condition for compound twin formation is that the shape deformation is a state of pure shear. Mechanistically, this is a necessary but not sufficient condition. In the type I limit, the Bevis-Crocker condition is automatically satisfied, since the engineering shear plane (distortion) plane is parallel to the low-index terrace. For a compound twin formed by slant dislocations, only deviatoric distortions are allowed, and in coordinate such that the deviatoric distortions are pure shears, but the above restriction is also necessary. Thus, in addition to the deviations from perfect classical twinning for type I twinning, Appendix A, there are deviations for type II twinning as well.

Another feature unique to slant TD mechanisms is as follows [174]. The same type II twin can often be formed by the motion of perfect dislocations instead of TDs on the $K_{2}$ plane. For the $f c c$ example in Figure 47, the motion of alternating perfect dislocations with Burgers vectors $\mathbf{A C}$ and $\mathbf{B C}$ on two of every three planes produces the same engineering shear and hence the same twin as three $\gamma \mathbf{D}$ TDs on the same 3 planes. For bcc, one perfect $1 / 2[111]$ dislocation on one of every three planes produces the same shear and hence the same twin as three 1/6[111] TDs.

Type II twins are rare, with a few early results for CuAlNi [175] and NiTi [176]. A later TEM study of NiTi [177] showed that the interface, while irrational, was near (111) with facets providing most of the rotation to the irrational plane. This is what would be expected with a large rotation $\varphi / 2$ as described above. In a study of type II twins in triclinic devitrite [178], the twin interface was also irrational but near (010). This would be consistent with the small rotation $\varphi / 2$ case. Some of these observations are for annealing twins. While the emphasis here is on deformation twins, the mechanism would apply for annealing twins, albeit formed with different rates of growth. 
Possibly, as described in [56] and [179] for transformations, the type II observations are related to the relative rates of nucleation $\dot{N}$ and growth $\dot{G}$ of pairs of TDs on a growing twin interface. If nucleation of TD pairs (loops) on the $K_{1}$ planes is difficult relative to the lateral velocity of the TDs, the spacing of TDs is relatively large. The TDs of like sign repel and the standard lenticular twin should form, Figure 49(a), with the aspect ratio increasing with an increase in the ratio $\dot{G} / \dot{N}$. The extensive terrace planes are $K_{1}$. For simple crystals such as metals, where e-cells have relatively small step heights, this is the expected case. Contrariwise, if growth were difficult, then the shape would resemble that in Figure 49(b). After partitioning, the terraces could form as in Figure 47, but the TDs move a relatively short distance compared to that in Figure 49(a). Now, the aspect ratio would increase with a decrease in the ratio $\dot{G} / \dot{N}$. In support of this concept, the structures where some type II twinning occurs are complex [178, 180], with correspondingly large t-cells and multiple shuffles, all of which should curtail $\dot{G}$.
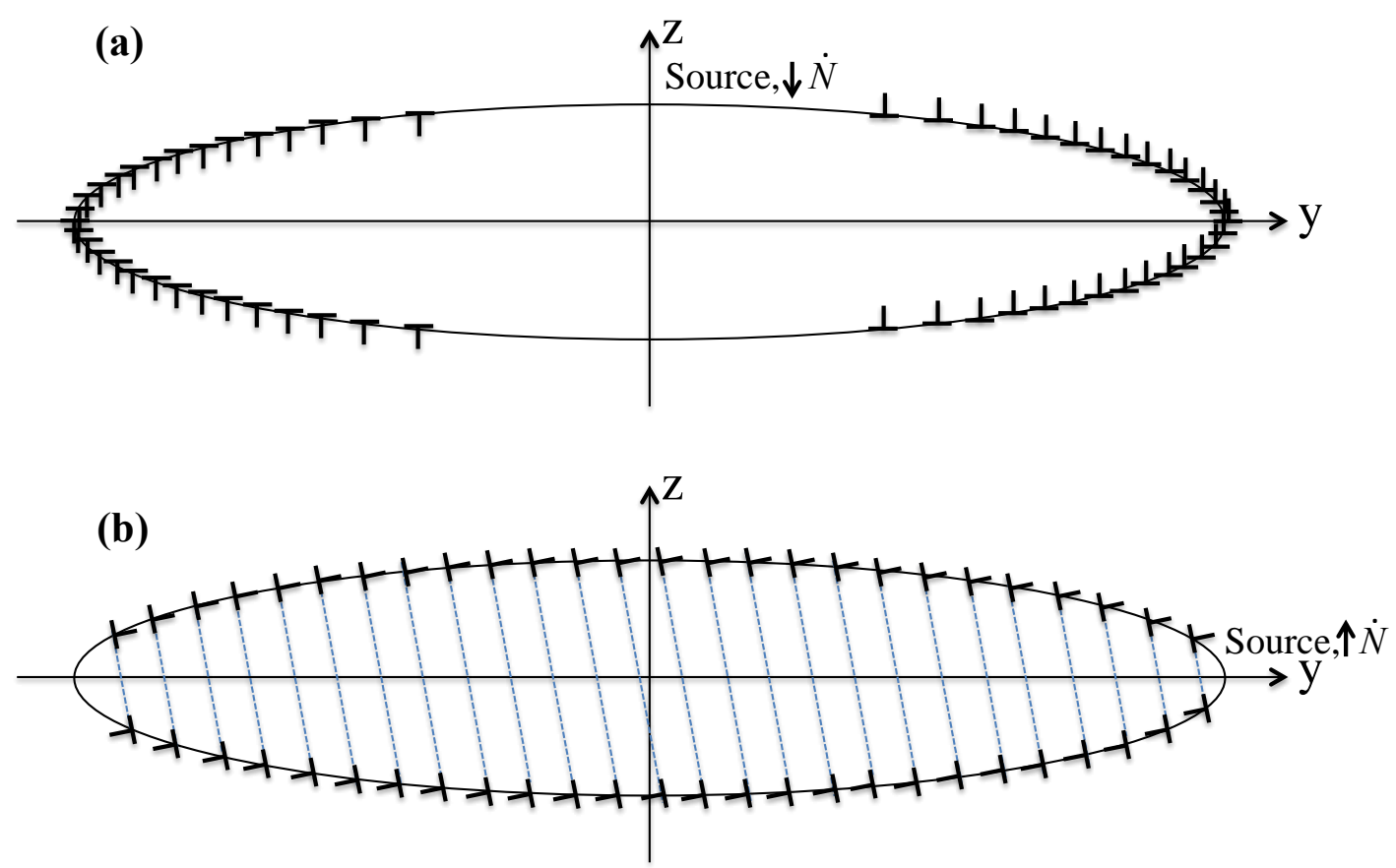

Figure 49. (a) Configuration with large nucleation rate $\dot{N}$ and low Growth rate $\dot{G}$ of TDs. (b) Low $\dot{N}$ and high $\dot{G}$. 
Table 1. Values of $\varphi$ and $\varphi^{0}$ for several $h c p$ metals. Here (1011) would be $K_{1}$ for a type I twin and $(\overline{1} 012)$ is the slant plane.

\begin{tabular}{|l|l|l|l|l|l|}
\hline Type & $c / a$ & $\theta^{0}$ & $\varphi^{0} / 2$ & $\square \square \square \square$ & $\square / 2$ \\
\hline $\mathrm{Cd}$ & 1.8859 & 72.10 & 8.95 & 0.170 & 4.90 \\
\hline $\mathrm{Zn}$ & 1.8563 & 71.99 & 9.00 & 0.139 & 3.98 \\
\hline $\mathrm{Co}$ & 1.6230 & 71.22 & 9.39 & 0.130 & 3.70 \\
\hline $\mathrm{Mg}$ & 1.6230 & 71.22 & 9.39 & 0.130 & 3.70 \\
\hline $\mathrm{Re}$ & 1.6150 & 71.19 & 9.40 & 0.140 & 4.03 \\
\hline $\mathrm{Zr}$ & 1.5930 & 71.14 & 9.43 & 0.168 & 4.83 \\
\hline $\mathrm{Ti}$ & 1.5873 & 71.12 & 9.44 & 0.175 & 5.03 \\
\hline $\mathrm{Hf}$ & 1.5810 & 71.10 & 9.45 & 0.183 & 5.27 \\
\hline Be & 1.5680 & 71.07 & 9.47 & 0.199 & 5.75 \\
\hline Ideal & 1.732 & 71.56 & 9.22 & 0 & 0 \\
\hline
\end{tabular}

\section{Multiscale modelling of deformation twinning in hcp metals}

The $h c p$ materials such as $\mathrm{Mg}, \mathrm{Zr}, \mathrm{Ti}$, and $\mathrm{Be}$ are important technologically [18183]. As a consequence it is relevant to optimize their mechanical and forming properties for specific applications. Hence, we need to understand the plasticity mechanisms at the nano and micrometer scale, as discussed in the previous sections, and employ these to develop physically based multi-scale models at the meso and macro scales [184-186].

\subsection{Plastic deformation in $h c p$}

The basic $h c p$ plasticity mechanisms are slip and twinning. The easiest dislocation slip is along the $\langle 11 \overline{2} 0\rangle$ close-packed directions on the (0001) basal or $\{10 \overline{1} 0\}$ prismatic planes $[187,188]$. These dislocations, however, do not generate deformation along the caxis and the alternative shear mechanisms available for doing so are $\langle\mathrm{c}+\mathrm{a}\rangle$ slip on $\{10 \overline{1} l\}$ planes and twinning. The $\langle\mathrm{c}+\mathrm{a}>$ dislocations have a large Burgers vector and a non-planar core that make their motion difficult. Tensile or compressive twins, on the other hand, are more easily activated, especially at intermediate or low temperature, and 
invariably at high deformation rates. Twinning in $h c p$ can take place on $\{10 \overline{1} 2\},\{10 \overline{1} 1\}$, $\{11 \overline{2} 2\}$ and $\{11 \overline{2} 1\}$ planes with TD's along $\langle 10 \overline{1} \overline{1}\rangle,\langle 10 \overline{1} \overline{2}\rangle,\langle 11 \overline{2} \overline{3}\rangle$ and $\langle\overline{1} \overline{1} 26\rangle$, respectively [187]. Which of these systems is preferentially active depends on the specific material, temperature, rate and - since twinning is directional with a unique sense of shear - upon the loading direction. The $\{10 \overline{1} 2\}$ tensile twin is prevalent in many $h c p$ metals.

Twin activation results in the formation of a localized-shear domain, where a large scale crystal reorientation takes place. As a consequence, several unique characteristics are associated with plasticity of $h c p$ materials. For example, rolled $h c p$ metals exhibit a characteristic texture component with the crystal c-axes (basal poles) preferentially aligned along the normal direction of the plate (Figure 50(a)). The flow stress evolution shows a strong anisotropy between the in-plane and through-thickness compression directions. In addition, the in-plane flow stress is very different in tension from that in compression (Figure 50(b)). Accompanying the reorientation of most grains as a consequence of twinning, the aggregate shows an increase in hardening rate and continuous evolution of grain microstructure with deformation (Figure 50(c)).

Several hardening models associated with slips have been proposed in the literature for $h c p$ metals [189-191]. Hardening models associated with twinning, however, are more challenging because of the complexity of twinning-related deformation events, which include nucleation and propagation of twins [37,100,138,139,192], detwinning [193-195], dislocation-twin interactions [196,197], twin-grain boundary interaction [198-102], and twin-twin interactions [165, 203-206]. The development of physics-based hardening models requires comprehensive understanding of mechanisms associated with the characteristics of twin-sheared domains, and structure and mobility of twinning-associated interfaces as discussed in previous Sections. Current model developments focus on describing nucleation and propagation of twins as two different mechanisms: while twin propagation is driven by long range stress states across grains, nucleation in $h c p$ is driven by local stress states and local atomistic configurations at grain boundaries. Corresponding to the stochastic nature of twinning associated with fluctuation of both internal stresses and local defect structure 
and content, we introduce statistics in twin modelling with the focus on twin nucleation. Here, we identify the available or missing computational procedures that allow us to introduce information about disconnections and defects associated with twin interfaces into constitutive models.

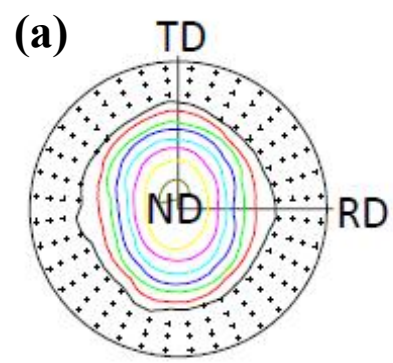

(0002) poles (b)

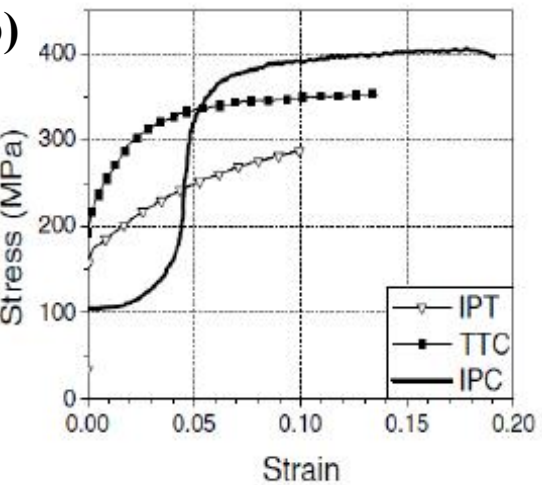

(c)

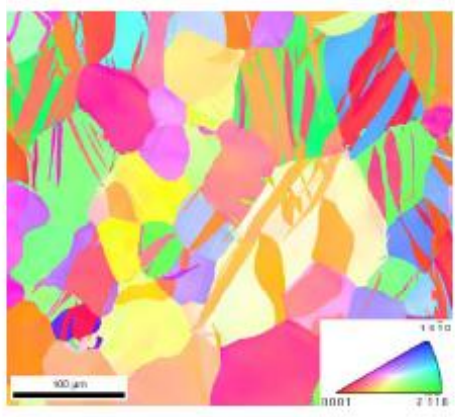

Figure 50. Mg AZ31 deformed at $300 \mathrm{~K}$ and $10^{-3} \mathrm{~s}^{-1}$. (a) Basal pole figure: distribution of the initial rolling texture showing texture component around the Normal Direction to the plate; (b) stress-strain response of samples deformed by In-Plane Tension, In-Plane Compression and Through Thickness Compression; (c) EBSD (electron back scattering diffraction) micrograph showing $\{10 \overline{1} 2\}$ tensile deformation twins.

\subsection{Modelling twinning in crystal plasticity models}

The ultimate aim in modelling plastic deformation of polycrystalline aggregates is to capture, at a macroscopic scale, the texture evolution (that determines anisotropy) and the associated hardening. A common feature of crystal plasticity models is that they represent the aggregate as a collection of variably oriented individual grains, each with an assigned volume fraction chosen to reproduce the measured initial texture. Deformation is accommodated by crystallographic slip and twin shear rates $\dot{\gamma}^{s}$ inside the grains. The total strain rate of the grain or a subdomain in it is given by the sum of shear rates contributed by all active slip and twinning systems (kinematic equation). Usually the shear rates are given by a visco-plastic power law of the resolved shear stress on the system.

$$
\dot{\varepsilon}_{i j}=\sum_{s} m_{i j}^{s} \dot{\gamma}^{s}=\dot{\gamma}_{0} \sum_{s} m_{i j}^{s}\left(\frac{m_{k l}^{s} \sigma_{k l}}{\tau^{s}}\right)^{n}
$$


Here $m_{i j}^{S}=0.5\left(n_{i}^{s} b_{j}^{S}+n_{j}^{S} b_{i}^{S}\right)$ is the geometric Schmid tensor for slip system $s, \sigma_{k l}$ is the average stress tensor inside the grain or material element, and $\tau^{S}$ is the threshold stress for activating the system. The variation of $\tau^{S}$ with strain determines the macroscopic hardening, and the distribution of $\dot{\gamma}^{s}$ determines the texture evolution of the aggregate. The threshold stress $\tau^{S}$ may include a rate and temperature dependence [189, 207].

Twinning localizes shear deformation in a volume fraction of the grain $\Delta f^{t w}=$ $\Delta \gamma^{t w} / S^{t w}$ ( $\Delta \gamma^{t w}$ is the shear strain contributed by the twinning system in the grain and $S^{t w}$ is the characteristic twin shear), instead of being homogeneously distributed as is the case with slip. In addition, the twinned volume is reoriented (with mirror symmetry across twin plane) with respect to the initial matrix. Correspondingly, most models treat the newly formed twin as having a new orientation independent of the parent and disregard the local interaction between the twin and its parent [208-211]. As the focus of modeling twinning was extended from reproducing observed deformation textures to also reproducing the stress-strain response of the aggregates, twinning induced hardening was introduced via latent hardening of slip due to a directional Hall-Petch effect arising from the (evolving) mean separation between twins inside the grain grains and the relative orientation of slip and twin planes [212,213], latent hardening of twinning dislocations caused by slip-created dislocations [189] and the transformation of glissile dislocations into sessile dislocations following the passage of the twin [214]. Twin-twin interaction induced hardening was not taken into account in these models.

The different approaches described above rely on the concept of some empirical threshold stresses that the local stress at the twin must meet in order for nucleation and propagation to take place. These processes have been treated phenomenologically, but the understanding of fundamental processes of twin nucleation, growth and interaction with other defects has reached the point where predictive input into constitutive modelling is possible, previous Sections, [189, 207-214]. We illustrate this in the ensuing Section.

12.3 Modelling nucleation and propagation of twins 
Proust et al [213] and Beyerlein and Tomé [189] recognize the need for assuming a nucleation stage for twinning in order to reconcile predictions with the experimental fact that twins are activated in a gradual fashion during deformation. Proust et al [213] introduce nucleation effects in $\mathrm{Mg}$ via an empirical law that delays nucleation until a certain amount of basal slip takes place. Beyerlein and Tomé [189] take a similar approach for $\mathrm{Zr}$, based on experimental characterization of $\{10 \overline{1} 2\}$ tensile twin evolution in $\mathrm{Zr}$ [215] which suggests that propagation is not feasible unless there is a previous accumulation of prismatic dislocations. Beyerlein and Tomé [189] assume a threshold stress $\tau_{n u c l}$ for nucleation higher than a threshold $\tau_{\text {prop }}$ for propagation and propose an empirical form of the probability $P\left(\sigma>\sigma_{\text {nucl }}\right)=1-\exp \left(-\rho^{\text {prism }} / \rho_{\text {saturat }}\right)$ for capturing the experimental evidence.

Reversal of the $\{10 \overline{1} 2\}$ twin (detwinning) plays an important role during strain path changes, such as those associated with strain cycling. Several aspects of twinning have been accounted for when modelling detwinning: Proust et al [213] speculate that there is no nucleation process associated with detwinning and that the threshold stress for detwinning is lower than the one for twin propagation. Aydiner et al [216] and Arul Kumar et al [217] document the existence of a back-stress associated with twin transformation, which would help in activating detwinning. Wang et al. [218, 219] rely on this experimental evidence to propose a model for twinning-detwinning and implement it in an Elasto-Visco-Plastic (E-VPSC) formalism [211]. Each and all of the above modification provide a more robust representation of the response of $h c p$ materials subjected to reloads. In all of these models nucleation and growth of the twin are characterized by a threshold stress and driven by the mean stress in either the parent or the twin domain.

\subsection{Models incorporating statistical variation}

The constitutive laws presented above for describing twinning do not account for specific twin nucleation mechanisms or for the statistical nature of twinning. Instead, the onset of twinning is modelled by a deterministic resolved shear stress criterion that tends to favour the twin variant with the highest Schmid factor in the grain. 
Twin nucleation, however, is energetically unlikely to occur inside the grain because it involves a zonal dislocation with multiple atomic layers according to molecular dynamics simulations [100, 138, 139]. Correspondingly, twin nucleation exhibits dependence on grain boundaries. Extensive experimental electron back scattering diffraction (EBSD) supports the notion that $h c p$ twins always start at grain boundaries [220-222]. Automated-image-analysis characterization of twins present in EBSD images was reported by [202, 223] for $\mathrm{Zr}$ and $\mathrm{Mg}$, respectively. A suite of twin statistics was produced which provides essential correlations between presence of twins, number of twins, twin thickness, twin type, twin variant and grain orientation, grain size, neighboring grains. Those twinning statistics provide crucial information (an example of which is shown in Figure 51) that guides the development of twin nucleation models and link microscale with mesoscale features.

In what follows we assume that nucleation always takes place at grain boundaries, and that it is statistically linked to local atomic structure and local stress concentrations. The statistical model and the procedure used for characterizing stress fluctuations at the grain boundaries are described in the next two sections.
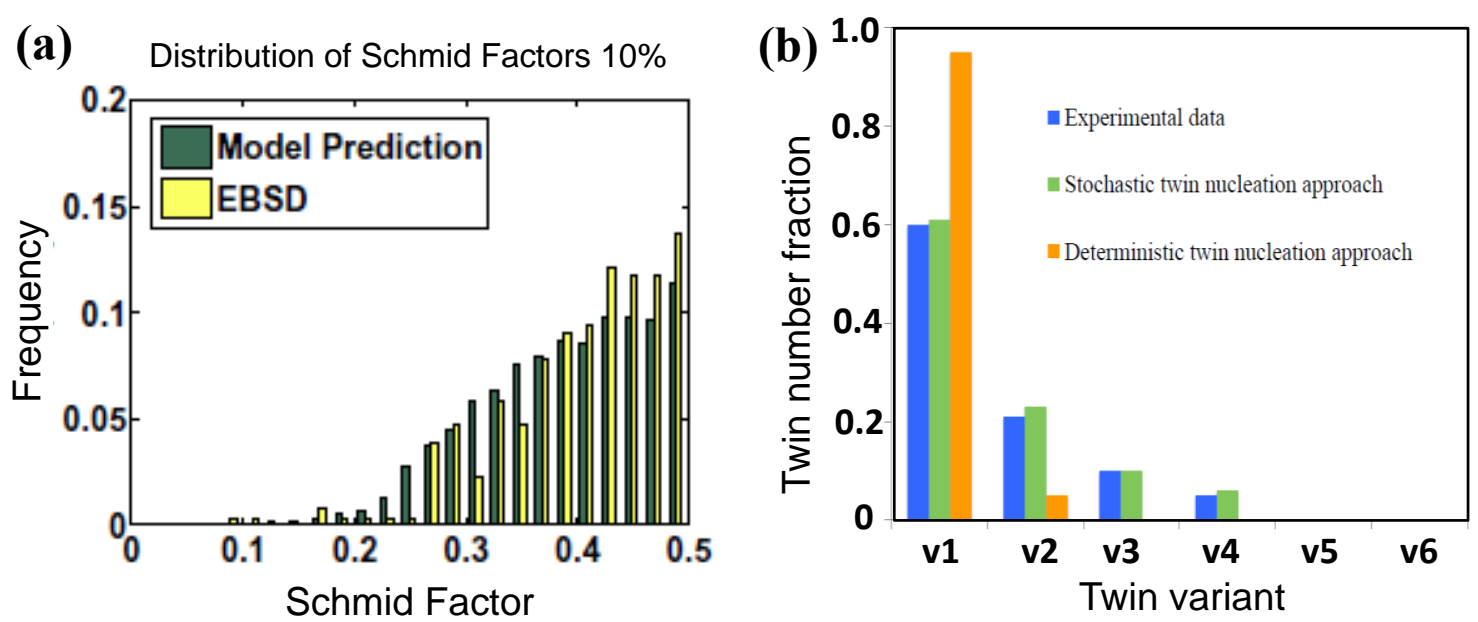

Figure 51. Twin statistics based on EBSD data of rolled Zr compressed $10 \%$ along the In Plane direction at 76K [202]. Comparison of experimental measurements and model predictions. (a) Distribution of twins as a function of their Schmid factors (SF); Frequency of twin variants as a function of their SF: v1 is the variant with the highest SF in a grain, and v6 the one with the lowest SF. 


\subsubsection{Stochastic model for twin nucleation}

The stochastic model for twin nucleation at grain boundaries is described in detail in $[224,225]$ and only the main concepts are reviewed here. Experimental studies provide evidence that in polycrystalline metals deformation twins predominantly nucleate from grain boundaries and interfaces [220, 221, 226]. As discussed in Section 6, grain boundaries are high defect density regions in a grain structure, containing misfit dislocations, ledges, stacking faults, and nano-voids, and thus providing numerous possible sources for twin nuclei. In addition, boundaries tend to develop relatively high local stresses [225, 227], which would be required to convert the grain boundaries defects into twin nuclei.

Molecular dynamics simulation studies of several grain boundary configurations [228, 229] suggest that high local stress induced by small pile-ups causes atoms at and in the vicinity of the dislocation core to shuffle into twin nuclei. Other nuclei formed within a characteristic region or area $a_{\mathrm{c}}$ of the boundary that has the same structure have the same orientation. Hence the growing nanoscale twins can coalesce, forming a larger twin. That is, one characteristic grain boundary area $a_{\mathrm{c}}$ produces one large crystalline twin, formed from many tinier grain boundary twin nuclei. The underlying assumptions adopted in this model are that twin nucleation occurs at grain boundaries and is controlled by the local defect configurations of grain boundaries (a material variable) and by the stress concentrations at grain boundaries (a mechanical variable) when the polycrystal is deformed. Both can vary statistically, giving rise to the heterogeneity in twinning observed experimentally.

Concerning the material component, in the stochastic model proposed for twin nucleation the critical stress for nucleation events follows a probability distribution that is influenced by grain boundary structure. According to the stochastic model, the probability $\mathrm{P}(\mathrm{S}<\tau)$ that the critical shear stress to nucleate a twin in $a_{\mathrm{c}}$ is less than or equal to $\tau \square$ is given by the following Weibull distribution: $P(S<\tau)=1-$ $\exp \left(-\left(\tau / \tau_{c}\right)^{\alpha}\right)$. The parameter $a_{\mathrm{c}}$ is an important model length scale, wherein all dissociation events in $a_{\mathrm{c}}$ lead to one twin. It sets the minimum twin spacing and the maximum number of twin lamellae that can form from a grain boundary of area $A_{k}$ as $n^{*}$ $=\mathrm{A}_{\mathrm{k}} / a_{\mathrm{c}}$. The material parameter $\square$ governs the dispersion in $\mathrm{S}$ and is linked to the type 
of defects in the grain boundary. To implement the above model into computational mechanics simulations, each grain is assigned $k$ nearest neighbors $(k=1 \ldots \mathrm{nf})$ and a shared grain boundary facet of area $A_{k}$ with each of them. The boundary facets are divided into areas of size $a_{\mathrm{c}}$ and each area is probed probabilistically to decide whether a twin nucleates, of which variant, and when in the deformation process.

As for the mechanical contribution, the average stress in each grain is given by the effective medium VPSC scheme, and is determined by the crystallographic orientation, the grain shape and orientation, hardening by slip and twinning within the grain, and interactions with the surrounding homogeneous medium. The stress state calculated, $\sigma^{g}$, is homogeneous throughout the grain. At the micro scale, however, there are spatial fluctuations in the stress $\Delta \sigma(x)$ that deviate from the 'average' value $\sigma^{g}$ and more so near the grain boundary. These fluctuations are particularly important for twinning. The local stress $\sigma^{l o c}=\sigma^{g} \pm \Delta \sigma(x)$ present in the vicinity of the grain boundary can impact when and where twins nucleate and which twin variants are selected. These stresses arise from numerous factors, such as interactions between dislocations and twins at grain boundaries, local defects, triple and quadruple junctions, alloying elements, etc., and are much harder to predict.

Fluctuations need to be treated as a statistical entity. To calculate the variance of the stress within a grain and the stress concentrations present at the grain boundary [227] recurred to the full-field three dimensional fast fourier transform (FFT) to calculate local stress fields [230, 231]. This method, based on representing the aggregate by means of a three dimensional Voronoi tessellation defining cells of crystal orientations, gives the spatially resolved stress fields. We process these to generate the stress distribution that develops near grain boundaries for a $\mathrm{Zr}$ polycrystal with rolling texture. We express the fluctuation of each stress component at the grain boundaries as a quasi-Gaussian function (shown in Figure 52). The stress fluctuations $\Delta \sigma$ turn out to be independent of texture, but increase with plastic deformation.

The simulation strategy that we use is the following. We use VPSC to calculate the grain stress $\sigma_{i j}^{g}$ and we randomly probe the statistical distribution to assign a fluctuation $\Delta \sigma_{i j}$ to each elementary area of each facet of the grain. Next, we calculate the 
local resolved shear in each twin variant $\tau^{l o c}(v)=m_{i j}^{v} \sigma_{i j}^{l o c}=\tau^{g}(v) \pm \Delta \tau(v)$ and assume that the variant most like to nucleate, denoted as $v_{\max }$, will be the one with the highest local value of resolved shear. We tag the latter twin variant and make it available for propagation during subsequent deformation steps, provided that the growth conditions are met, as we discuss in the next Section.

We foresee using the same philosophy and a similar approach for characterizing intragranular stress fluctuations which may drive the mechanisms of twin growth discussed in Sections 4, 5 and 8. Alternatively, Discrete Dislocation Dynamics may provide such distributions with an even sharper scale resolution and increased dispersion than the FFT approach [232].

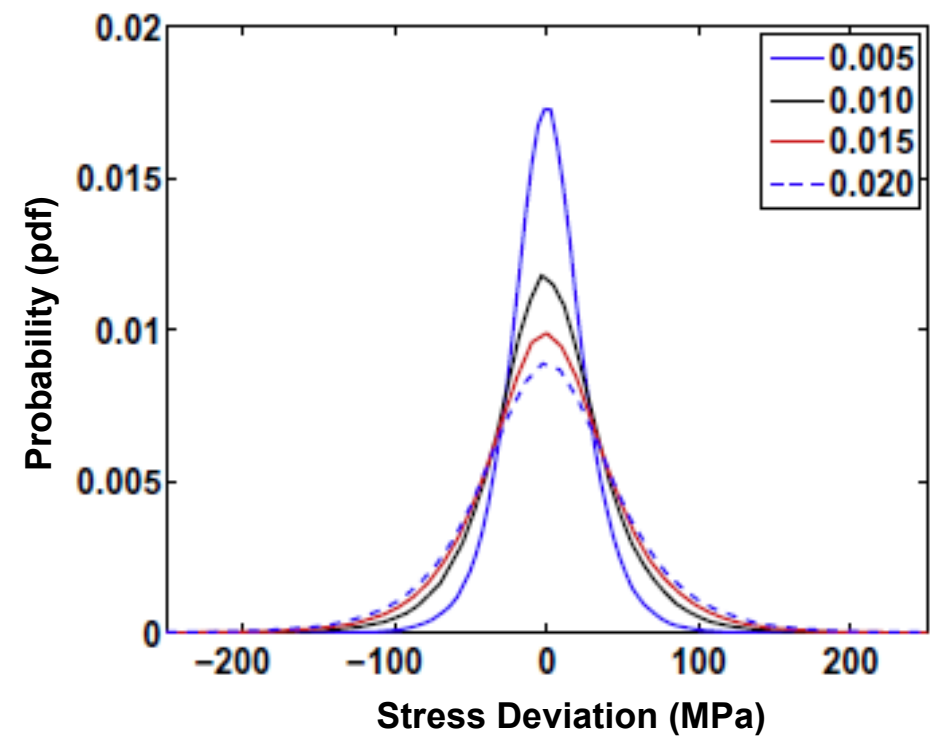

Figure 52. Distribution of the local stress component $\sigma_{11}$ calculated for $\mathrm{Zr}$ using the FFT scheme [232]. Different distributions correspond to macroscopic strains of 0.5, 1.0, 1.5 and $2.0 \%$.

\subsubsection{Stress associated with twin growth}

Twin variants that are nucleated by the probability model are allowed to extend across the grain and grow or, more specifically, expand in thickness and accommodate shear strain. From the discussion in Sections 4, 5, and 8, it is apparent that the models for twin propagation should include a coupling with the dislocation model. The reason is that 
dislocations reactions at grain boundaries and twin boundaries drives twin nucleation and propagation, respectively.

The stress state that drives growth is considered to be the long-range average stress state calculated by VPSC. Specifically, the grain level resolved shear stress is used to drive twin propagation of the twin variant statistically nucleated. Mimicking the approach used for slip, it is usual to consider a threshold stress that the resolved shear stress needs to overcome in order to activate twinning, and to express the shear rate as a power law of the resolved shear.

Because twin growth is governed by the long-range stresses in the grain and twin nucleation by the short-range stress states at the grain boundaries, the crystallographic orientation of the grain affects twin growth more strongly than twin nucleation. One important issue concerns the grain level resolved shear stress to use in the model for activating twin growth. As we discuss in Section 12.1, the stress that activates twinning dislocations is the one acting at the twin interface [218, 219]. Such stress exhibits different levels of complexity, usually buried under a single empirical value for the grain level resolved shear stress. First, the back-stress induced by the twinning transformation modifies the stress field in the vicinity of the twin and should be taken into account, as demonstrated by $[217,233]$. Second, incompatibility at grain boundaries imposes the need for plastic accommodation of the localized twin shear, which also induces a reaction stress upon the twin [217, 234]. Clearly, at the smaller length scale, local stress fields associated with dislocations will also produce fluctuations on the twin interface, and will locally affect the reaction processes.

As an example, we reproduce some results reported previously by [225] for rolled Zr. These results are meant to demonstrate that when the probability model for twin nucleation is implemented into crystal plasticity models the multi-scale framework predicts specific features of the macroscopic behaviour that would not be captured otherwise. In the simulations that follow the stress tensor of the parent was used for driving twin growth, and back stress, neighbour or grain size effects upon twinning are not included. 
We examine the deformation response of rolled pure $\mathrm{Zr}$ when compressed along the Rolling Direction. Figure 53(a) compares the predicted stress-strain curves at $76 \mathrm{~K}$, $150 \mathrm{~K}$ and $300 \mathrm{~K}$ with the experimental curves for $\mathrm{Zr}$. The initial plateau in hardening rate at $76 \mathrm{~K}$ and $150 \mathrm{~K}$ is the signature of twinning being activated. The subsequent increase in hardening rate comes from barriers posed by twins to dislocation slip, and from hard-to-deform twin reorientated domains. In addition, the predicted total twin fractions in Figure 53(b) are consistent with EBSD measurements of twins.
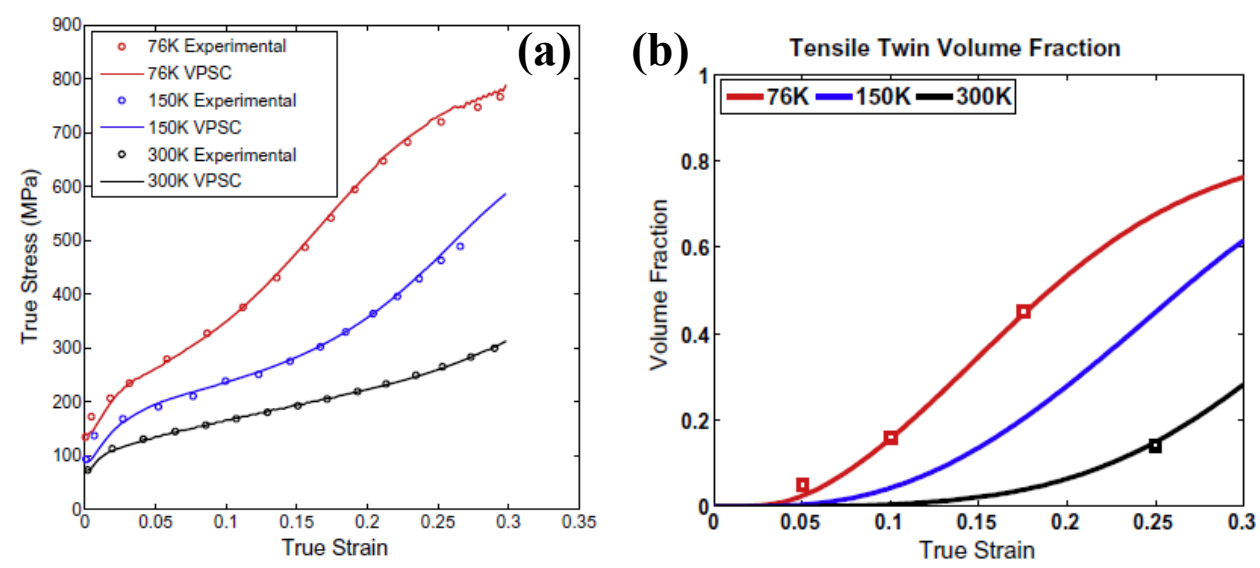

Figure 53. High purity Zirconium. (a) Comparison of model predictions with experimental stress-strain curves for in-plane compression at 76, 150, 300K; (b) comparison of $\{10 \overline{1} 2\}$ twin volume fraction predicted by the model with measurements made by EBSD [225].

Predicting the right proportion of twin variants proves crucial to capturing the details of the hardening and texture evolution. Figure 51(b) shows that the stochastic twin nucleation model correctly predicts the observed proportion of twin variants. A nonstochastic approach based on a Schmid criterion for nucleation and propagation overwhelmingly favours variant 1 , while a stochastic nucleation approach shows that variant 2 to 4 twins provide a non-negligible contribution to deformation (Figure 51(b)). As a consequence, texture evolution in hcp metals takes place in a more gradual and diffuse manner. Texture evolution in $h c p$ metals is sensitive to the volume fraction of twinned material and the twin variants selected. As strain increases, the volume fraction of twinned material increases. Within the twinned regions, the basal poles are reoriented by $\sim 86^{\circ}$ towards the direction of loading. The corresponding measured and predicted deformation textures for $\mathrm{Zr}$ are shown in Figure 54 [224]. Measurement and model 
consistently show that reorientation of the basal poles along the compression axis as a result of $\{10 \overline{1} 2\}$ twinning is not apparent at $4 \%$, starts being present at $9 \%$ and becomes significant at $14 \%$ strain and higher. Because slip modes are harder to activate at low temperatures the twinning contribution to deformation increases at low temperatures.

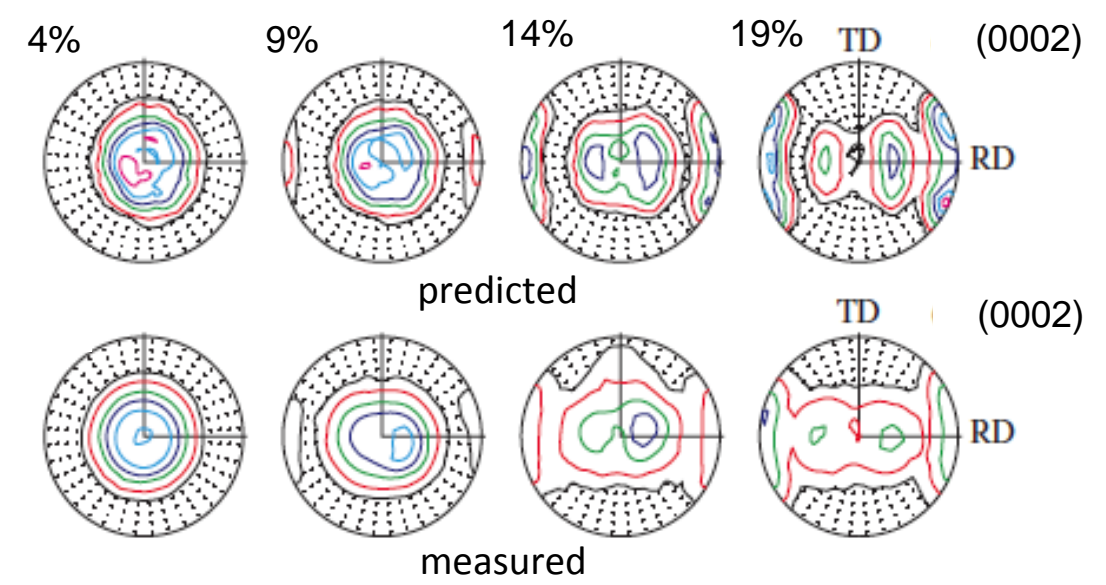

Figure 54. Comparison of predicted and measured basal pole figures for $\mathrm{Zr}$ deformed at $76 \mathrm{~K}$ at different amounts of strain for in-plane compression along the TD [224]. Contour lines are $0.5,1,2,3,4,5,6,8$ m.r.o. Notice the large twin-related reorientation of the c-axis.

An important observation is that twins nucleate long before they start making a substantial impact on the stress-strain response and texture, a feature that is captured by the stochastic approach. Model predictions show that at 5\% strain, twins have already nucleated in $\sim 60 \%$ of the grains, although the influence of twinning on bulk properties does not become apparent until the macroscopic flow stress increases to the values required for growing the less favourable twin variants.

The models described in this Section and the results presented are meant to show how the physical micro-scale mechanisms of twinning discussed in this work may in the future be implemented in constitutive models and how, although being microscopic, they may have a clear and measurable effect on the macroscopic response of aggregates.

\section{Discussion}

As discussed in [3], a number of early works treated step-type defects. The formal definition of such defects and disconnections in the TM formalized these concepts and 
provided precise definitions of $\boldsymbol{b}$ and $h$, with emphasis on CDPs. The TM linked the topological theory [1] with mechanics descriptions and circuit concepts [2-5]. Analogously, there were early works that related to definitions of shuffles $[32,58,108]$. Here we formalize these in terms of e-cells [104], which relate to a formal description of shuffle vectors $\boldsymbol{s}$. As shown here, the e-cells are useful in the description of a variety of twinning defects, including disconnections, facets, incoherent interfaces, disclinations, synchroshear defects and domain defects. These descriptions are consistent with the trend in the topological theory of starting with a CDC of maximum symmetry and introducing a series of symmetry-breaking operations to produce a given interface $[1,130]$. There have been other innovative applications of the TM to describe disclinations and other related defects $[116,117]$ that agree qualitatively with the present work. These used NDCs instead of CDCs and unnecessarily introduced a trichromatic complex, and hence do not agree quantitatively or conceptually with the TM as described here [2-7].

Also, agreeing with other works [104, 120, 235, 236], the descriptions here differ from the "shuffle-dominated" models in [237-239]. The situation is clearly demonstrated in $[104,120]$. In the shuffle-dominated models, either terraces move normal to themselves by pure shuffle, or the interface has no terraces and moves by pure shuffle. The former is refuted, because all observations and simulations of interface motion, e.g. $[104,235]$ show that the mechanism is one of disconnections moving on a terrace plane and that there is an accompanying deformation defined by $\boldsymbol{b}$. The second model would apply to some massive transformations with incoherent interfaces [240-242] which would move by pure shuffle displacements $[62,236]$ : we know of no such mechanism for twinning. Also, defects moving by pure steps, discussed in Sect. 3-5, would move by pure shuffle, in most cases glide-shuffle [62,108,236]. However, as specifically demonstrated in HRTEM [97], the mechanism is still one of a pure-step disconnection moving on a terrace. Finally, the result in [97] showing a nucleus formed by pure shuffle has been cited as supporting the shuffle-dominated model [239]. This is not so, since the nucleus is coherent as is standard in nucleation theory, Sect 6, and subsequently grows by disconnection transit. 
The treatment of incoherent interfaces is consistent with general observations [11, 32-34, 242-244]. The formation and structure of this interface focuses on disconnection mechanisms and the relation to facets. The new concept discussed here is the possibility of the injection of emissary dislocations into the twin. There has been little consideration of the structure of the plane of shear $S$, and that relates to the wrap-around model [152]. Here, the alternative of twist disclination accommodation is presented. In simulation studies [151], the observed interface is faceted and contains facets of both types of model.

The terraces in the TM correspond to type I classical interfaces. When residual disconnections or facets remain on the boundary, as for a typical lenticularly-shaped deformation twin, the mean interface obviously deviates from the $K_{1}$ plane. Twin boundaries formed by slant TDs agrees with the classical picture only in having a $K_{2}$ plane in general. Only in special cases such as for $\{111) f c c$ twins, are all four twinning elements rational: a compound twin. The TM predicts in the general case that the $K_{1}$ plane should have or be near a low index orientation, consistent with experimental observations [32-34]. A key feature in this analysis is that the rotation accompanying the type II twin shear is partitioned to the two crystals.

The last comment relates to a general difference between the TM and either the PTMC [23-25] or O-lattice models [57, 245], as discussed in [172, 173]. For mode I type transformations, the rotation accompanying the associated engineering shear is included in all of these models. However, if there is a $b_{\mathrm{z}}$ component to the disconnection Burgers vector, there is an added rotation (tilt wall) $[92,93]$. The added rotation associated with the tilt wall is partitioned in the TM but not in the other models, so the latter do not precisely describe $\boldsymbol{b}$ or the interface structure. For type-I twins, there is no $b_{z}$ so all three models agree. However for many type II twins and for facets such as P/B facets, the partitioning must be considered. There is a way to unify these models. If the O-lattice and PTMC models were applied to a doubly coherent crystal, that is one where the difference in interplanar $d$ spacings parallel to the terrace plane, the models would again agree. Such a procedure is essentially that in the near-O lattice method [246]. Then one could consider separately the rotation accompanying the tilt rotation produced by the relaxation of coherency normal to the terrace plane and partition it, and the other models 
would still agree with the TM. In a small angle approximation usually accurate, one can just take the orientation relationship angle determined in the other models and partition half of it to the parent crystal. When rotations are larger, the more exact partitioning procedure in the TM is necessary. There are other advantages of the TM: coherence in the z-direction is unnecessary; exact vector quantities are given in the CDP, CDC, and, when rotation is involved, in the RCDP and RCDC; and defects associated with symmetry operations other than translation are directly determined.

The smearing of discrete disconnections into a continuous infinitesimal array produces the ideal Bilby bicrystal. Through the e-cells this provides atomic scale results for the displacements accompanying twinning as manifested by $\boldsymbol{b}, \boldsymbol{s}$ and $\boldsymbol{p}$. This is an analog of the more general application to phase transformations [27, 28, 247]. This smearing, when applied to deformation twins or their pileup representations as in Figure D-1, provides a link to the macroscopic level in multiscale modeling. Thus the constitutive models in Sect. 12 link back to disconnections and other structures at the nanoscale level.

There are other thermodynamic or kinematic effects that relate to the defects discussed here, but are either peripheral to the present description of structure, or remain difficult to analyze energetically. The former includes solute adsorption to interfaces and individual defects; solute drag; and pinning effects. A notable example of the first effect is the observation of core solute adsorption to disconnections in Mg alloys [248]. An example of the drag effect is the formation energy, activation energy for motion, and general kinetics of kink nucleation and propagation at disconnections. Kinks and kink motion have been observed on disconnections [46, 249]. However the 3D, non-periodic nature of kinks makes the calculation of their energetics difficult.

\section{Summary}

We have provided an analysis of twinning in terms of the topological model, describing twinning disconnections in terms of step heights h, Burgers vectors $\boldsymbol{b}$, shuffle vectors $\boldsymbol{s}$ and rigid displacements of crystals $\boldsymbol{p}$ relative to one another at the interface. A convenient reference for these disconnection properties is the exchange-cell derived from 
the coherent dichromatic complex. Some new concepts are introduced concerning the relation to classical twin descriptors, to emissary dislocations, to synchroshear, to all three types of twin interfaces, and to type II twinning. A constitutive model relates these microscopic properties to the macroscale.

\section{Acknowledgments}

The authors were fully supported by the Office of Basic Energy Sciences, Project FWP 06SCPE401, under US DOE Contract No W-7405-ENG-36. The authors are indebted to R.C. Pond for helpful discussion and contributions to this work. J.W. Christian, R.G. Hoagland, J. M. Howe, S. Mahajan, and Y.T. Zhu also provided helpful discussions and comments on this topic.

\section{References}

1. Pond RC. Line defects in interfaces. In: Nabarro FRN, editor. Dislocations in Solids, 1989;8:5-66. Amsterdam: Elsevier.

2. Pond RC, Hirth JP. Defects at surfaces and interfaces. Solid State Phys 1994;47:287-365.

3. Howe JM, Pond RC, Hirth JP. The role of disconnections in phase transformations. Prog. Mater. Sci. 2009;54:792-838.

4. Hirth JP, Pond RC. Compatibility and accommodation in displacive phase transformations. Prog. Mater. Sci. 2011;56:586-636.

5. Hirth JP, Pond RC, Hoagland RG, Liu XY, Wang J. Interface defects, reference spaces and the Frank-Bilby equation. Prog. Mater. Sci. 2013;58:749-823.

6. Hirth JP, Pond RC. Steps, dislocations and disconnections as defects relating to structure and phase transformations. Acta Mater. 1996;44:4749-4763.

7. Pond RC, Ma X, Chai YW, Hirth JP. Topological modelling of martensitic transformations. In: Nabarro FRN, Hirth JP, editors. Dislocations in solids 2007;13:225-262. Amsterdam: Elsevier.

8. Hirth JP. Dislocations, steps and disconnections at interfaces. J. Phys. Chem. Solids 1994;55:985-989.

9. Lehmann O, Flussige Kristalle. Leipzig, 1904.

10. Friedel G. Les états mésomorphes de la matière. Ann. Phys. (Paris) 1922;18:273-473.

11. Frank FC. Crystal dislocations-Elementary concepts \& definitions. Philos Mag 1951;42:809-819. 
12. De Wit R. Theory of disclinations: II. Continuous and discrete disclinations in anisotropic elasticity. J Res Nat Bur Stand: Phys Chem A 1973;77:49-100.

13. Nabarro FRN. In: Simmons JA, deWit R, Bullough R, edtors. Fundamental Aspects of Dislocation Theory, Spec pub 317. Washington DC, Nat Bur Stand; 1970. P. 710.

14. Serra A, Bacon DJ. A new model for $\{10 \overline{1} 2\}$ twin growth in hcp metals. Philos Mag A 1996;73:333-343.

15. Li YJ, Chen YJ, Walmsley JC, Mathinsen RH, Dumoulin S, Roven HJ. Faceted interfacial structure of $\{1011\}$ twins in Ti formed during equal channel angular pressing. Scripta Mater 2010;62:443-446.

16. Kim I, Kim J, Shin DH, Liao XZ, Zhu YT. Deformation twins in pure titanium processed by equal channel angular pressing. Scripta Mater 2003;48:813-817.

17. Frank FC, Van der Merwe JH. One-dimensional dislocations. I. Static theory, Proc. R. Soc. London A 1949;198:205-216.

18. Frank FC. In: Report of the symposium on the plastic deformation of crystalline solids. Pittsburgh: Carnegie Institute of Technology; 1950. p. 150-154.

19. Olson GB. Interphase Kinematics and the Roles of Structure and Composition in Solid-State Transformations. Scripta Metall 1987;21:1023-1028.

20. Howe JM and Smith DA. Comparison between the invariant line and structural ledge theories for predicting the habit plane, orientation relationship and interphase boundary structure of plate-shaped precipitates. Acta Metall Mater 1992;40:2343-2350.

21. Aaronson HI, Howe JM, Hall MG, Furuhara T and Hirth JP. Mobility of structural ledges. Scripta Mater 1997;37:1301-1307.

22. Dahmen U. Surface relief and the mechanism of a phase transformation. Scripta Metall 1987;21:1029-1034.

23. Wechsler MS, Lieberman DS and Read TA. On the theory of the forma of martensite, Trans. AIME, 1953;197:1503-1529.

24. Bowles JS, Mackenzie JK. The crystallography of martensite transformations, part I. Acta Metall 1954;2:129-137.

25. Wayman CM. Introduction to the crystallography of martensitic transformations. New York: Macmillan; 1964.

26. Khater HA, Serra A, Pond RC, Hirth JP. The disconnection mechanism of coupled migration and shear at grain boundaries. Acta Mater 2012;60:2007-2020.

27. Bilby BA. In: Report of the conference on defects in crystalline solids. London: Physical Soc; 1955. p. 124.

28. Bullough R, Bilby BA. Continuous distributions of dislocations: surface dislocations and the crystallography of martensitic transformations. Proc Phys Soc Lond B 1956;69:1276-1286. 
29. Frank FC. Martensite. Acta Metall 1953;1:15-21.

30. Geisler AH. Crystallography of phase transformations. Acta Metall 1953;1:260281.

31. Bilby BA. On the mutual transformation of lattices. Philos Mag 1953;44:782-785.

32. Christian JW, Mahajan S. Deformation Twinning. Prog Mater Sci 1995;39:1-157.

33. Knowles KM. In: Kelly A, Groves GW, Kidd P editors. Crystallography and Crystal defects. New York:Wiley;2000, p. 315

34. Zhu YT, Liao XZ, Wu XL. Deformation twinning in nanocrystalline materials. Prog Mater Sci 2012;57:1-62.

35. Pond RC, Vlachavas DS. Bicrystallography. Proc Roy Soc London A 1983;386:95-143.

36. Pond RC, Serra A, Bacon DJ. Dislocations in interfaces in the hep metals-II. Mechanisms of defect mobility under stress. Acta mater 1999;47:1441-1453.

37. Wang J, Liu L, Tomé CN, Mao SX, Gong SK. Twinning and de-twinning via glide and climb of twinning dislocations along serrated coherent twin boundaries in hexagonal-close-packed metals. Mater Res Lett 2013;1:81-88.

38. Serra A, Pond RC, Bacon DJ. Computer-simulation of the structure and mobility of twinning dislocations in hcp metals. Acta Metall Mater 1991;39:1469-1480.

39. Wang J, Beyerlein IJ, Hirth JP. Nucleation of elementary $\{\overline{1} 011\}$ and $\{\overline{1} 013\}$ twinning dislocations at a twin boundary in hexagonal close-packed crystals. Model Simu Mater Sci Eng 2012;20:024001.

40. Ostapovets A, Serra A. Characterization of the matrix-twin interface of a (1012) twin during growth. Philos Mag 2014;94:2827-2839.

41. Narayan J. Recent progress in thin film epitaxy across the misfit scale. Acta Mater 2013;61:2703-2724.

42. Christian JW, Crocker AG. Dislocations and lattice transformations. In: Nabarro FRN, editor. Dislocations in Solids 1980;3:165-250. Amsterdam: Elsevier.

43. Narayan J, Larson BC. Domain epitaxy: A unified paradigm for thin film growth. J Appl Phys 2003;93:278-285.

44. Young TD, Kioseoglou J, Dimitrakopulos GP, Dłuzewski P, Komninou P. 3D modelling of misfit networks in the interface region of heterostructures. J Phys D: Appl Phys 2007;40:4084-4091.

45. Olson GB, Cohen M. Dislocation theory of martensitic transformations. In: Nabarro FRN, editor. Dislocations in Solids 1986;7:295-403. Amsterdam: Elsevier.

46. Howe JM, Dahmen U, Gronsky R. Atomic mechanisms of precipitate plate growth. Philos Mag A 1987; 56:31-61. 
47. Mahon GJ, Howe JM, Mahajan S. HRTEM investigation of the $\{225\}$ Austenite/Martensite interface in an Fe-8Cr-1C Alloy. Philos Mag Lett 1989;59:273-279.

48. Howe JM, Benson WE. Determination of the Atomic Structure, Mechanisms and Kinetics of Precipitate Growth in Solids by In Situ Hot-Stage High-Resolution Transmission Electron Micrsocopy. Interface Sci 1995;2:347-363.

49. Pond RC, Sarrazit F. Diffusive fluxes associated with interfacial defect motion and interaction. Interface Sci 1996;4:99-118.

50. Chen IW, Chiao YH. Theory and experiment of martensitic nucleation in ceramics and ferrous alloys. Acta Metall 1985;33:1827-1845.

51. Aaronson HI. In: Zackay VF, Aaronson HI, editors. Decomposition of austenite by diffusional processes. New York: Wiley Interscience; 1962. p. 387.

52. Read WT Jr. Dislocations in Crystals. New York: McGraw-Hill;1953.

53. Pond RC, Medlin DL, Serra A. A study of the accommodation of coherency strain by interfacial defects at a grain boundary in gold. Philos Mag 2006;86:4667-4684.

54. Ashby MF, Spaepen F, Williams S. The structure of grain boundaries described as a packing of polyhedral. Acta Metall 1978;26:1647-1663.

55. Klassen-Neklyudova MV. Mechanical twinning of crystals. New York: Consultants Bureau; 1964.

56. Bollmann W. Crystal defects and crystalline interfaces. Berlin: Springer-Verlag; 1970.

57. Zhang WZ. Decomposition of the transformation displacement field. Philos Mag 1998;78:913-933.

58. Bilby BA, Crocker AG. The theory of the crystallography of deformation twinning. Proc Roy Soc Lond A 1965;288:240-255.

59. Kurtz RJ, Hoagland RG, Hirth JP. Computer simulation of extrinsic grainboundary defects in the Sigma $11,<101>\{131\}$ symmetric tilt boundary. Philos Mag 1999;79:683-703.

60. Merkle KL, Thompson LJ, Phillipp F. Thermally activated step motion observed by high-resolution electron microscopy at a (113) symmetric tilt grain-boundary in aluminium. Philos Mag Lett 2002;82:589-597.

61. Gibbs JW. Thermodynamics. New York: Dover: 1961.

62. Hirth JP, Lothe J. Theory of Dislocations. 2nd ed. Melbourne Fl: Krieger; 1992.

63. Pond RC. The measurement of excess volume at grain boundaries using transmission electron microscopy. J Microscopy 1979;116:105-114.

64. Kumar A, Wang J, Tomé CN. First-principles study of energy and atomic solubility of twinning-associated boundaries in hexagonal metals. Acta Mater 2015;85:144-154.

65. Howe JM. Interfaces in materials, New York: Wiley; 1997. 
66. Freund LB, Suresh S. Thin film materials. Cambridge: Cambridge University Press; 2003.

67. Hirth JP, Pond RC. Strains and rotations in thin deposited films. Philos Mag 2010; 90:3129-3147.

68. Pond RC, Vitek V. Periodic Grain Boundary Structures in Aluminium. I. A Combined Experimental and Theoretical Investigation of Coincidence Grain Boundary Structure in Aluminium. Proc Roy Soc Lond A 1977;357:453-470.

69. Cai W, Bulatov VV, Chang JP, Li J, Yip S. Dislocation core effects on mobility. In: Nabarro FRN, Hirth JP, Eds. Dislocations in Solid 2004:12(64):1-80. Amsterdam: Elsevier.

70. Medlin DL, Carter CB, Angelo JE, Mills Mj. Climb and glide of $a / 3\langle 111\rangle$ dislocations in an aluminium $\Sigma=3$ boundary. Philos Mag 1997;75A:733-747.

71. Medlin DL, Foiles SM and Carter CB. Grain boundary dislocation structure and motion in an Aluminum $\sum=3$ [011] Bicrystal. Mater. Res. Soc. Symp. Proc. 1996;466:125-130.

72. Bacmann JJ, Silvestre G, Petit M, Bollmann W. Partial secondary dislocations in germanium grain boundaries. I. Periodic network in a $\Sigma=5$ coincidence boundary. Philos Mag A 1981;43:189-200.

73. Smalc-Koziorowska J, Tsiakatouras, Lotsari A, Georgakilas A, Dimitrakopulos GP. The defect character of GaN growth on r-plane sapphire. J Appl Phys 2010;107:073525.

74. Hirth JP, Wells RG. Disclination Structures in Bloch Wall Lattices in BaFe12O19 and SmCo5. J Appl Phys 1970;41:5250-5254.

75. Hahn T, editor. International Tables for Crystallography. New York: Wiley;2014.

76. Pond RC. On the Characterisation of Interfacial Defects Using High Resolution Electron Microscopy. Interface Sci 1995;2:299-310.

77. Chou YT, Li JCM, Proc. symposium on the mathematical theory of dislocations. New York: ASME;1969. P.116.

78. Laub T, Eshelby JD. The velocity of a wave along a dislocation. Philos Mag 1966;14:1285-1293.

79. Li JCM. Disclination model of high angle grain boundaries. Surf Sci 1972;31:1226.

80. Akarapu S, Zbib HM, Hirth JP. Modeling and analysis of disconnections in tilt walls. Scripta Mater 2008;59:265-267.

81. Romanov A. Disclinations in crystalline solids. In: Nabarro FRN, editor. Dislocations in Solids 1992:9:191-422. Amsterdam: Elsevier.

82. Dimitrakopulous GP, Karakostas T, Pond RC. The defect character of interface junction lines. Interface Sci 1996;4:129-138. 
83. Iijima S. Fine particles of silicon. II. decahedral multiply-twinned particles. Jap J Appl Phys 1987;26:365-372.

84. Dimitrakopulos G, Komninou Ph, Karakostas Th, Pond RC. Junction line disclinations: characterisation and observations. Interface Sci 1999;7:217-229.

85. Bouret A, Lasalmonie A, Naka S. In-situ high resolution observation of hydride precipitation in titanium. Scripta Metall 1986;20:861-866.

86. Hirth JP, Pond RC, Lothe J. Disconnections in tilt walls. Acta Mater 2006;54:4237-4245.

87. Amelinckx S, In: Fisher JC et al, editors. Dislocations and Mechanical Properties of Crystals. New York:Wiley;1957, p. 3.

88. Pond RC, Cherns D. Surface structure and the origin of $1 / 4<111\rangle$ interfacial dislocations in $\mathrm{NiSi}_{2} / \mathrm{Si}$ epitaxial films. Surface Sci 1985;152-153:1197-1202.

89. Komninou P, Dimitrakopulous GP. Interfacial dislocations at the junction lines of $\{211\}$ microfacets of a twin boundary in silicon. Philos Mag 1998;78A:255-272.

90. Wang J, Huang H. Novel deformation mechanism of twinned nanowires. Appl Phys Lett 2006;88:203112.

91. Aaronson HI and Hall MG. A history of the controversy over the roles of shear and diffusion in plate formation above $\mathrm{Md}$ and a comparison of the atomic mechanisms of these processes. Metall Mater Trans A 1994;25:1797-1819.

92. Christian JW. Crystallographic theories, interface structures, and transformation mechanisms. Metall Mater Trans A 1994;25:1821-1839.

93. Hirth JP. Ledges and dislocations in phase transformations. Metall Mater Trans A 1994;25:1885-1894.

94. Enomoto M, Hirth JP. Computer simulation of ledge migration under elastic interaction. Metall Mater Trans A 1996;27:1491-1500.

95. Sleeswyk AW. Emissary dislocations: theory and experiments on the propagation of deformation twins in a-iron. Acta Metall 1962;10:705-725.

96. Wasilewski RJ. Surface distortions in twinned niobium (Columbium) crystals. Metall Trans 1970;1:1617-1622.

97. Li N, Wang J, Misra A, Zhang X, Huang JY, Hirth JP. Twinning dislocation multiplication at a coherent twin boundary. Acta Mater 2011;59:5989-5996.

98. Wang J, Beyerlein IJ, Hirth JP, Tomé CN. Twinning dislocations on $\{\overline{1} 011\}$ and $\{\overline{1} 013\}$ planes in hexagonal close-packed crystals. Acta Mater 2011;59:39904001.

99. Dash S, Brown N. An investigation of the origin and growth of annealing twins. Acta Metall 1963;11:1067-1075.

100. Wang J, Hirth JP, Tomé CN. (1 012$)$ Twinning nucleation mechanisms in hexagonal-close-packed crystals. Acta Mater 2009;57:5521-5530. 
101. Rosenbaum HS. In Reed-Hill RE, Hirth JP, Rogers HC, editors. Deformation Twinning. New York: Gordon and Breach:1964, p. 42.

102. Bristowe PD, Crocker AG. A computer simulation study of the structure of twinning dislocations in body centred cubic metals. Acta Metall 1977;25:13631371.

103. Braisaz T, Ruterana P, Nouet G, Pond RC. Investigation of $\{10 \overline{1} 2\}$ twins in $\mathrm{Zn}$ using high-resolution electron microscopy: Interfacial defects and interactions. Philos Mag A 1997;75:1075-1095.

104. Pond RC, Hirth JP, Serra A, Bacon DJ. Atomic displacements accompanying deformation twinning: shears and shuffles. Mater Res Lett (2016). http://dx.doi.org/10.1080/21663831.2016.1165298

105. Khater HA, Serra A, Pond RC. Atomic shearing and shuffling accompanying the motion of twinning disconnections in Zirconium. Philos Mag 2013;93:1279-1298.

106. Kiho H. The Crystallographic Aspect of the Mechanical Twinning in Ti and $\alpha-\mathrm{U}$. J Phys Soc Japan 1958;13:269-272.

107. Jaswon MA, Dove DB. The crystallography of deformation twinning. Acta Cryst 1960;13:232-240.

108. Bevis M, Crocker AG. Twinning modes in lattices. Proc Roy Soc Lond A 1969;313:509-529.

109. Christian JW. The theory of transformations in metals and alloys. Oxford: Pergamon; 2002.

110. Ackland GJ, Wooding SJ, Bacon DJ. Defect, surface and displacement-threshold properties of alpha-zirconium simulated with a many-body potential. Philos Mag A 1995;71:553-565.

111. Thornton PH. Reply to discussion 'on $\{10.1\}$ twinning in the hep structure'. Acta Metall 1966;14:444-445.

112. Wang J, Li N, Anderoglu O, Zhang X, Misra A, Huang JY, Hirth JP. Detwinning mechanisms for growth twins in face-centered cubic metals. Acta Mater 2010;58:2262-2270.

113. Liu BY, Wang J, Li B, Lu L, Zhang XY, Shan ZW, Li J, Jia CL, Sun J, Ma E. Twinning-like lattice reorientation without a crystallographic twinning plane. Nature Commun 2014;5:3297.

114. Sun Q, Zhang XY, Tu J, Ren Y, Qin H, Liu Q. Characterization of basal-prismatic interface of $\{1012\}$ twin in deformed titanium by high-resolution transmission electron microscopy. Philos Mag Lett 2015;95:145-151.

115. Tu J, Zhang X, Wang J, Sun Q, Liu Q, Tomé CN, HRTEM characterization of $\{10 \overline{1} 2\}$ twin boundaries in cobalt. Appl Phys Lett 2013;103:051903.

116. Barrett CD, EI Kadiri H. Impact of deformation faceting on and embryonic twin nucleation in hexagonal close-packed metals. Acta Mater 2014;70:137-161. 
117. Barrett CD, EI Kadiri $\mathrm{H}$. The roles of grain boundary dislocations and disclinations in the nucleation of $\{10 \overline{1} 2\}$ twinning. Acta Mater 2014;63:1-15.

118. Kohn W, Sham LJ. Self-consistent equations including exchange and correlation effects. Phys Rev 1965;140:A1133-A1138.

119. Fullman RL, Fisher JC. Formation of annealing twins during grain growth. J Appl Phys 1951;22:1350-1355.

120. Ostapovets A, Burgit A, Groger R. Deformation due to migration of faceted $\{10 \overline{1} 2\}$ twin boundaries in magnesium and cobalt. Philos Mag 2015;85:41064117.

121. Le Lan A, Dubertret A. A development of Kronberg's model for $\{10 \overline{1} 2\}$ twins in H.C.P. metals. Extension to $\{11 \overline{2} 2\}$ twins. Phys Stat Sol 1979;51:497-507.

122. Westlake DG. On $\{10.1\}$ twinning in the h.c.p. structure. Acta Metall 1966;14:442-444.

123. Serra A, Bacon DJ, Pond RC. The crystallography and core structures of twinning dislocations in h.c.p. metals. Acta Metall 1988;12:3183-3203.

124. Kamat SV, Hirth JP, Müllner P. The effect of stress on the shape of a blocked deformation twin. Philos Mag A 1996;73:669-680.

125. Sun Q, Zhang XY, Ren Y, Tu J, Liu Q. Interfacial structure of $\{10 \overline{1} 2\}$ twin tip in deformed magnesium alloy. Scripta Mater 2014;90:41-44.

126. Liu BY, Wan L, Wang J, Ma E, Shan Z. Terrace-like morphology of the boundary created through basal-prismatic transformation in magnesium, Scripta Mater 2015;100:86-89.

127. Russell KC, Aaronson HI. Sequences of precipitate nucleation. J Mater Sci 1975;10:1991-1999.

128. Olson GB, Cohen M. A general mechanism of martensitic nucleation: Part I. general concepts and the FCC-HCP transformation. Metall Trans A 1976;7:18971904.

129. Matthews JW, Blakeslee AE. Defects in epitaxial multilayers: II. Dislocation pileups, threading dislocations, slip lines and cracks. J Cryst Growth 1975;32:273280.

130. Sutton AP, Balluffi RW. Interfaces in crystalline materials. Oxford: Oxford University Press; 1995.

131. Pond RC, Bollmann W. The symmetry and interfacial structure of bicrystals. Phil Trans Roy Soc (London) 1979;A292:449-472.

132. Mahajan S, Chin GY. Formation of deformation twins in f.c.c. crystals. Acta Metall 1973;21:1353-1363.

133. Byerlein IJ, Tomé CN. A probabilistic twin nucleation model for HCP polycrystalline metals. Proc Roy Soc Lond A 2010;466:2517-2544. 
134. Tschopp MA, McDowell DL. Grain boundary dislocation sources in nanocrystalline copper. Scripta Mater 2008;58:299-302.

135. Van Swygenhoven H, Derlet PM, Froseth AG. Stacking fault energies and slip in nanocrystalline metals. Nature Mater 2004;3:399-403.

136. Wu XL, Liao XZ, Srinivasan SG, Zhou F, Lavernia EJ, Valiev RZ, Zhu YT. New deformation twinning mechanism generates zero macro-strain in nanocrystalline metals. Phys Rev Lett 2008;100:095701.

137. Zhu YT, Narayan J, Hirth JP, Mahajan S, Wu XL, Liao XZ. Formation mechanisms of single and multiple deformation twins in FCC materials. Acta Mater 2009;57:3763-3770.

138. Wang J, Yadav SK, Hirth JP, Tomé CN, Beyerlein IJ. Pure-shuffle nucleation of deformation twins in Hexagonal-Close-Packed metals. Mater Res Lett 2013;1:126-132.

139. Wang J, Hoagland RG, Hirth JP, Capolungo L, Beyerlein IJ, Tomé CN. Nucleation of a (1012) twin in hexagonal close-packed crystals. Scripta Mater 2009;61:903-906.

140. Wang J, Yu Q, Jiang Y, Beyerlein IJ. Twinning-associated boundaries in hexagonal-close-packed metals. JOM 2014;66(1):95-101.

141. Kronberg ML. Plastic deformation of single crystals of sapphire: Basal slip and twinning. Acta Mater 1957;5:507-524.

142. Hazzledine PM, Pirouz P. Synchroshear transformations in Laves phases. Scripta. Metal 1993;28:1277-1282.

143. Chu F, Pope DP. In: Yoo MH, Wuttig M, editors. Twinning in Advanced Materials. Warrendale, PA: TMS;1984, p. 15.

144. Wang J, Misra A, Hirth JP. Shear response of $\Sigma 3\{112\}$ twin boundaries in facecentered-cubic metals. Phys Rev B 2011;83:064106.

145. Gleiter H, Hornbogen E, Baro G. The mechanism of grain boundary glide. Acta Metall 1968;16:1053-1060.

146. Hirth JP. The influence of grain boundaries on mechanical properties. Metall Trans 1972;3(12):3047-3067.

147. Sleeswyk AW, Verbraak CA. Twin-slip interaction in f.c.c. crystals._Acta Metall 1961;9:917-927.

148. Sleeswyk AW. Emissary dislocations: twin interaction and twin growth. Acta Metall 1964;12(7):669-672.

149. Zhu YT, Wu XL, Liao XZ, Narayan J, Kecskes LJ, Mathaudhu SN. Dislocationtwin interactions in nanocrystalline fcc metals. Acta Mater 2011; 59(2):812-821.

150. Wang J, Beyerlein IJ, Tomé CN. Reactions of lattice dislocations with grain boundaries in $\mathrm{Mg}$ : Implications on the micro scale from atomic-scale calculations. Int J Plasticity 2014;56:156-172. 
151. Liu Y, Li N, Shao S, Gong MY, Wang J, McCabe RJ, Jiang Y, Tomé. Characterizing the boundary lateral to the shear direction of deformation twins in magnesium. Nature Commu 2016;7:11577.

152. Hull D. The initiation of slip at the tip of a deformation twin in $\alpha$-iron. Acta Metall 1961;9:909-912.

153. Wang J, Anderoglu O, Hirth JP, Misra A, Zhang X. Dislocation structures of $\Sigma 3\{112\}$ twin boundaries in face centered cubic metals. Appl Phys Lett 2009;95:021908.

154. Livingston JD, Chalmers B. Multiple slip in bicrystal deformation. Acta Metall 1957;5:322-327

155. Carter CB, Medlin DL, Angelo JE and Mills MJ. The 112 lateral twin boundaries in FCC metals. Mater Sci Forum 1995;207-209 (1):209-212.

156. Campbell GH, Chan DK, Medlin DL, Angelo JE and Carter CB. Dynamic observation of the FCC to $9 \mathrm{R}$ shear transformation in a Copper $\sum=3$ incoherent twin boundary. Scripta Mater. 1996;35(7): 837-842.

157. Wolf U, Ernst F, Muschik T, Finnis MW, Fischmeister HF. The influence of grain boundary inclination on the structure and energy of $\Sigma 3$ grain boundaries in Copper. Philos Mag A 1992;66:991-1016.

158. Hofmann D, Ernst F. Quantitative high-resolution transmission electron microscopy of the incoherent $\Sigma 3(211)$ boundary in $\mathrm{Cu}$. Ultramicroscopy 1994;53:205-221.

159. Rittner JD, Seidman DN, Merkel KL. Grain-boundary dissociation by the emission of stacking faults. Phys Rev B 1996;53:R4241-R4244.

160. Medlin DL, Campbell GH, Carter CB. Stacking defects in the 9R phase at an incoherent twin boundary in copper. Acta Mater 1998;46:5135-5142.

161. Wang J, Li N, Misra A. Structure and stability of $\Sigma 3$ grain boundaries in face centered cubic metals. Philos Mag 2013;93:315-327.

162. Hirth JP. Stabilization of strained multilayers by thin interlayers. J Mater Res 1993;8:1572-1577.

163. Amelinckx A, Dekeyser W. The structure and properties of grain boundaries. Solid State Phys 1959;8:325-499.

164. Yu Q, Wang J, Jiang Y, McCabe RJ, Tomé CN. Co-zone $\{\overline{1} 012\}$ twin interaction in magnesium single crystal. Mater Res Lett 2013;2:82-88.

165. Yu Q, Wang J, Jiang Y, McCabe RJ, Li N, Tomé CN. Twin-twin Interactions in Magnesium. Acta Mater 2014;77:28-42.

166. Babyak WJ, Rhines FN. The relationship between the boundary area and hardness of recrystallized cartridge brass. Trans Amer Inst Min Met Engr 1960; 218:21-23. 
167. Field RD, Brown DW, Thoma DJ. Texture development and deformation mechanisms during uniaxial straining of U-Nb shape-memory alloys. Philos Mag 2005;85:1441-1457.

168. Patterson RL, Wayman CM. The crystallography and growth of partially- twinned martensite plates in Fe-Ni alloys. Acta Metall 1966;14(3):347-369.

169. Clarke WAT, Wagoner RH, Shen ZY, Lee TC, Robertson IM, Birnbaum HK. On the criteria for slip transmission across interfaces in polycrystals. Scripta Metall Mater 1992;26:203-206.

170. Cahn RW. Plastic deformation of a-uranium: twinning and slip. Acta Metall 1953;1:49-70.

171. Hirth JP, Pond RC. Strains and rotations in thin deposited films. Philos Mag 2010;90:3129-3147.

172. Kelly PM. In: Olson GB, Lieberman DS, Saxena A, editors. Proceedings of ICOMAT 2008. Warrendale PA: TMS;2010, p. 119.

173. Hirth JP, Pond RC. In: Olson GB, Lieberman DS, Saxena A, editors. Proceedings of ICOMAT 2008. Warrendale PA: TMS;2010, p. 123.

174. Bullough, R. Deformation twinning in the diamond structure. Proc Roy Soc Lond A 1957;241(1227): 568-577.

175. Ichinose S, Funatsu Y, Otsuka K. Type II Deformation Twinning in g1' Martensite in Cu-Al-Ni Alloys. Acta Metal 1985;33:1613-1620.

176. Knowles KM, Smith DA, The crystallography of the martensitic transformation in equiatomic nickel-titanium. Acta Metall 1981;29:101-110.

177. Xie ZL, Liu Y. HRTEM study of $\langle 011\rangle$ type II twin in NiTi shape memory alloy. Philos Mag 2004;84:3497-3507.

178. Knowles KM, Ramsey CNF. Type II twinning in devitrite $\mathrm{Na}_{2} \mathrm{Ca}_{3} \mathrm{Si}_{6} \mathrm{O}_{16}$. Philos Mag Lett 2012;92:38 $\square 48$.

179. Hirth JP. In Fundamental aspects of dislocation theory. Simmons JA, deWit R, Bullough R. Editors. Nat Bur Stand Spec Pub 317:1970; p.547

180. Li B, Knowles KM, Molecular dynamics simulation of albite twinning and pericline twinning in low albite. Model Simul Mater Sci Eng 2013;21:055012.

181. Mathaudhu SN, Nyberg EA. Magnesium technology: Magnesium alloys in U.S. military applications: Past, Current and future solutions. Essential Readings in Magnesium Technology, 2010. p. 71-76.

182. Ezugwu EO, Wang ZM. Titanium alloys and their machinability - a review. J Mater Proc Tech 1997;68(3): 262-274.

183. Benedict M, Levi H, Pigford T. Nuclear chemical engineering. Nuclear Science and Engineering (United States), 1982. 82(4). 
184. Horstemeyer MF. Integrated Computational Materials Engineering (ICME) for metals: using multiscale modeling to invigorate engineering design with science. John Wiley \& Sons, 2012.

185. Tomé CN, Beyerlein IJ, Wang J, McCabe RJ. A multi-scale statistical study of twinning in magnesium. JOM 2011;63(3):19-23.

186. Wang J, Beyerlein IJ, Tome CN. An atomic and probabilistic perspective on twin nucleation in Mg. Scripta Mater 2010;63(7): 741-746.

187. Yoo MH. Slip, twinning, and fracture in hexagonal close-packed metals. Metall Trans A 1981;12(3): 409-418.

188. Yoo MH, Morris JR, Ho KM, Agnew SR. Nonbasal deformation modes of HCP metals and alloys: role of dislocation source and mobility. Metall Mater Trans A 2002;33(3): 813-822.

189. Beyerlein IJ, Tomé CN. A dislocation-based constitutive law for pure $\mathrm{Zr}$ including temperature effects. Int J Plasticity 2008;24:867-895.

190. Brown DW, Beyerlein IJ, Sisneros TA, Clausen B, Tomé CN. Role of twinning and slip during compressive deformation of beryllium as a function of strain rate. Intl J Plasticity 2012;29:120-135.

191. Tomé CN, Canova GR, Kocks UF, Christodoulou N, Jonas JJ. The relation between macroscopic and microscopic strain hardening in FCC polycrystals. Acta Metall 1984;32:1637-1653.

192. Leclercq L, Capolungo L, Rodney D. Atomic-Scale Comparison Between Twin Growth Mechanisms in Magnesium. Mater Res Lett 2014; 2: 152-159.

193. Wu L, Jain A, Brown DW, Stoica GM, Agnew AR, Clausen B, Fielden DE, Liaw PK. Twinning-detwinning behavior during the strain-controlled low-cycle fatigue testing of a wrought magnesium alloy, ZK60A. Acta Mater 2008;56:688-695.

194. Wang YN, Huang JC. The role of twinning and untwinning in yielding behavior in hot-extruded Mg-Al-Zn alloy. Acta Mater 2007;55:897-905.

195. Yu Q, Zhang J, Jiang Y. Direct observation of twinning-detwinning-retwinning on magnesium single crystal subjected to strain-controlled cyclic tensioncompression in [0001] direction. Philos Mag Lett 2011; 91:757-765.

196. Yin SM, Yang HJ, Li SX, Wu SD, Yang F. Cyclic deformation behavior of asextruded Mg-3\%Al-1\%Zn. Scripta Mater 2008;58:751-754.

197. Serra A, Bacon DJ, Pond RC. Twins as barriers to basal slip in Hexagonal-ClosePacked Metals. Metall Mater Trans A 2002;33:809-812.

198. Xu S, Schuman C, Lecomte J. Accommodative (10-12) twins at high angle grain boundaries inrolled pure titanium. Scripta Mater 2016;116:152-156.

199. Kacher J, Minor AM. Twin boundary interactions with grain boundaries investigated in pure rhenium. Acta Mater 2014:81:1-8.

200. Wang L, Yang Y, Eisenlohr P, Bieler TR, Crimp MA, Mason DE. Twin nucleation by slip transfer across grain boundaries in commercial purity titanium. 
Metall Mater Trans A. 2010:41(2):421-430.

201. Bieler TR, Wang L, Beaudoin AJ, Kenesei P, Lienert U. In situ characterization of twin nucleation in pure Ti using 3D-XRD. Metall Mater Trans A 2014;45:109122.

202. Capolungo L, Marshall PE, McCabe RJ, Beyerlein IJ, Tome CN. Nucleation and growth of twins in Zr: a statistical study. Acta Mater 2009;57(20):6047-6056.

203. Roberts E, Partridge PG. The accommodation around $\{10 \overline{1} 2\}<\overline{1} 011>$ twins in magnesium. Acta Metall 1966;14:513-527.

204. El Kadiri H, Kapil J, Oppedal AL, Hector Jr LG, Angew AR, Cherkaoui M, Vogel SC. The effect of twin-twin interactions on the nucleation and propagation of $\{10 \overline{1} 2\}$ twinning in magnesium. Acta Mater 2013; 6:3549-3563.

205. Beyerlein IJ, Wang J, Barnett MR, Tomé CN. Double twinning mechanisms in magnesium alloys via dissociation of lattice dislocations. Proc Roy Soc Lond A 2012;468:1496-1520.

206. Sun Q, Zhang XY, Ren Y, Tan L, Tu J. Observations on the intersection between $\{10 \overline{1} 2\}$ twin variants sharing the same zone axis in deformed magnesium alloy. Mater Characterization 2015;109:160-163.

207. Follansbee PS, Kocks UF. A constitutive description of the deformation of copper based on the use of the Mechanical Threshold Stress as an internal state variable. Acta Metall 1988;36:81-93.

208. Van Houtte P. Simulation of the rolling and shear texture of Brass by the Taylor theory adapted for mechanical twinning. Acta Metall 1978;26:591-604.

209. Tomé CN, Lebensohn RA, Kocks UF. A model for texture development dominated by deformation twinning: application to zirconium alloys. Acta Metall Mater 1991;39:2667-2680.

210. Kalidindi SR. Incorporation of deformation twinning in crystal plasticity models, J Mech Phys Solids 1998;46:267-290.

211. Wang H, Wu PD, Tomé CN, Huang Y. A finite strain elasto-viscoplastic selfconsistent model for polycrystalline materials. J Mech Phys Solids 2010;58:594612.

212. Proust G, Tomé CN, Kaschner GC. Modeling texture, twinning, and hardening evolution during deformation of hexagonal materials. Acta Mater 2007;55:21372148.

213. Proust G, Tomé CN, Jain A, Agnew SR. Modeling the effect of twinning and detwinning during strain path changes of $\mathrm{Mg}$ alloy AZ31. Int J Plasticity 2009;25:861-880.

214. Oppedal AL, El Kadiri H, Tomé CN, Kaschner GC, Vogel SC, Baird JC, Horstemeyer MF. Effect of dislocation transmutation on modeling hardening mechanisms by twinning in magnesium. Int J Plasticity 2012;30-31:41-61. 
215. McCabe RJ, Cerreta EK, Misra A, Kaschner GC, Tomé CN. Effects of texture, temperature and strain on the deformation modes of zirconium. Philos Mag A 2006;86:3595-3611.

216. Aydiner CC, Bernier JV, Clausen B, Lienert U, Tomé CN, Brown DW. Evolution of stress in individual grains and twins in a magnesium alloy aggregate. Phys Rev B 2009;80:024113.

217. Kumar MA, Kanjarla AK, Niezgoda SR, Lebensohn RA, Tomé CN. Numerical study of the stress state of a deformation twin in magnesium. Acta Mater 2015;84:349-358.

218. Wang H, Wu PD, Wang J, Tomé CN. A crystal plasticity model for HCP crystals including twinning and detwinning mechanisms. Intl J Plasticity 2013;49:36-52.

219. Wang H, Wu PD, Tomé CN, Wang J. A constitutive model of twinning and detwinning for HCP polycrystals. Mats Sci Eng A 2012;555:93-98.

220. Wang L, Eisenlohr P, Yang Y, Bieler TR, Crimp MA. Nucleation of paired twins at grain boundaries in Titanium. Scripta Mater 2010;63:827-830.

221. Wang L, Barabash R, Bieler TR, Liu W, Eisenlohr P. Study of \{11-21\} Twinning in $\alpha$-Ti by EBSD and Laue Microdiffraction. Metall Mater Trans A 2013;44:3664-3674.

222. Nervo L, King A, Fitzner A, Ludwig W, Preuss M. A study of deformation twinning in a titanium alloy by X-ray diffraction contrast tomography. Acta Mater 2016;105:417-428.

223. Beyerlein IJ, Capolungo L, Marshall PE, McCabe RJ, Tomé CN. Statistical analyses of deformation twinning in magnesium. Philos Mag 2010;90:2161-2190.

224. Beyerlein IJ, Tomé CN. A probabilistic twin nucleation model for HCP polycrystalline metals. Proc Roy Soc Lond A 2010;466:2517-2544.

225. Niezgoda SR, Kanjarla AK, Beyerlein IJ, Tomé CN. Stochastic model of twin nucleation in polycrystals: an application to hexagonal close packed metals. Intl $\mathrm{J}$ Plasticity 2014;56:119-138.

226. Adams BL, Fullwood DT, Basinger JA, Hardin T. High Resolution EBSD-Based Dislocation Microscopy. Mater Sci Forum 2012;702-703:11-17.

227. Rollett AD, Lebensohn RA, Groeber M, Choi Y, Li J, Rohrer GS. Stress Hot Spots in Viscoplastic Deformation of Polycrystals. Model Simul Mater Sci Eng 2010;18:074005.

228. Wang J, Beyerlein IJ. Atomic structures of symmetric tilt grain boundaries in hexagonal close packed (hcp) crystals. Model Simul Mater Sci Eng 2012;20: 024002 .

229. Wang J, Beyerlein IJ, Atomic structures of [0110] symmetric tilt grain boundaries in hexagonal close-packed (hcp) crystals. Metall Mater Trans A 2012;43(10):3556-3569. 
230. Lebensohn RA. N-site modeling of a 3D viscoplastic polycrystal using Fast Fourier Transform. Acta Mater 2001;49:2723-2737.

231. Lebensohn RA, Kanjarla AK, Eisenlohr P. An elasto-visco-plastic formulation based on Fast Fourier transforms for the prediction of micromechanical fields in polycrystalline materials. Int J Plasticity 2012;32-33:59-69.

232. Balogh L, Capolungo L, Tomé CN. On the measure of dislocations densities from diffraction line profiles: a comparison with discrete dislocation methods. Acta Mater 2012;60:1467-1477.

233. Clausen B, Tomé CN, Brown DW, Agnew SR. Reorientation and stress relaxation due to twinning: modeling and experimental characterization for $\mathrm{Mg}$. Acta Mater 2008;56:2456-2468.

234. Abdolvand H, Wilkinson AJ. Assessment of residual stress fields at deformation twin tips and the surrounding environments. Acta Mater 2016;105:219-231.

235. Serra A, Bacon DJ, Pond RC. Comment on "Atomic Shuffling Dominated Mechanism For Deformation Twinning In Magnesium”. Phys Rev Lett 2010;104:029603.

236. Ostapovets A, Molnár P. On the relationship between the "shuffling-dominated" and "shear-dominated" mechanisms for $\{10-12\}$ twinning in magnesium. Scripta Mater 2013;69:287-290.

237. Li B, Ma X. Atomic Shuffling Dominated Mechanism for Deformation Twinning in Magnesium. Phys Rev Lett 2009;10:035503

238. Li B, Ma E. Li and Ma Reply. Phys Rev Lett 2010;104:029604

239. Li B, Zhang Y. Global Strain Generated by Shuffling-Dominated. Scripta Mater 2013;71:45-48.

240. Massalski TB. Massive transformations. Mater Sci Eng 1976;25:119-125

241. Aaronson HI. Mechanisms of the massive transformation. Metall Mater Trans A 2002;33A:2285-2297.

242. Massalski TB, Soffa WA, Laughlin, DE. The Nature and Role of Incoherent Interphase Interfaces in Diffusional Solid-Solid Phase Transformations. Metall Mater Eng A 2006;37:825-831.

243. Reed-Hill R, Hirth JP, Rogers HC. Deformation twinning. New York: Gordon and Breach;1964.

244. Yoo MH, Wuttig M. Twinning in advanced materials. TMS, Warrendale, PA, 1994.

245. Zhang WZ, Yang XP. Identification of singular interfaces with $\Delta \mathrm{gs}$ and its basis of the O-lattice. J Mater Sci 2011;46:4135-4156.

246. Miyano V, Ameyama K, Weatherly GC. Three Dimensional Near-Coincidence Site Lattice Modeling of $\alpha / \beta$ Interface Boundary Structure in Two Phase Titanium Alloy. ISIJ International. 2000;40(Suppl):S199-S203 
247. Bilby BA, Bullough R, Smith E. Continuous distributions of dislocations: a new application of the methods of non-riemannian geometry. Proc Roy Soc Lond A 1995;231:263-273.

248. Nie JF, Zhu YM, Liu JZ, Fang XY. Periodic segregation of solute atoms in fully coherent twin boundaries. Science 2013;340:957-960.

249. Wu HC, Kumar A, Wang J, Bi XF, Tomé CN, Zhang Z, Mao SX. Rollinginduced face centered cubic titanium in hexagonal close packed titanium at room temperature. Sci. Rep. 2016;6:24370.

250. Hirth JP, Balluffi RW. On grain boundary dislocations and ledges. Acta Metall 1973;21:929-942.

251. Cahn RW. Twinned crystals. Advan Phys 1954;3:363-445.

252. Klein C, Hurlbut CS. Manual of mineralogy, 20 ${ }^{\text {th }}$ edition. New York:Wiley. 1993.

253. Wang $\mathrm{H}, \mathrm{Wu} \mathrm{PD}$, Wang J. Modeling inelastic behavior of magnesium alloys during cyclic loading-unloading. Int J Plasticity 2013;47:49-64.

254. Mitchell TE, Hirth JP. The shape, configuration and stress field of twins and martensite plates. Acta Metall Mater 1991;39:1711-1717.

255. Eshelby JD. The force on an elastic singularity. Philos Trans Roy Soc Lond A 1951;244:87-112. 


\section{Appendix A. The Pond topological theory of bicrystal crystallography}

Crystal symmetry has important implications for displacive transformations such as twinning, and is applicable at three structural levels: (i) individual interfacial defects, (ii) individual plate-shaped products (variants), and (iii) assemblies of variants. These structural levels are topologically linked through the Principle of Symmetry Compensation [1], and one can enumerate the three categories systematically using the methods developed for Bicrystallography [35]. Formally, one begins with the space groups of the lattices of the matrix (black, $\mu$ ) and product (white, $\lambda$ ) crystals: so their combined crystallography is dichromatic. Much of this initial symmetry is broken when the product phase is embedded in the matrix. Symmetry can increase in forming a coherent pattern or complex from a natural dichromatic pattern (NDP), but then systematically decreases in proceeding from the commensurate/coherent dichromatic pattern (CDP) to the commensurate/coherent dichromatic complex (CDC) to the ideal bicrystal to the real bicrystal, Each stage of dissymmetrization can be compensated by the creation of a characteristic topological discontinuity. The focus here is on individual defects: variants entailing groups of defects are also considered, as treated elsewhere [1, $4,35]$, and as treated via the Frank-Bilby equation in [5].

The topological nature of line defects in continua was first developed by Volterra, who showed that dislocations and disclinations are characterized by translation and rotation operations, respectively [75]. For the case of single crystals, the Volterra operations characterizing perfect defects must be proper symmetry operations of the underlying crystal (even though those symmetries are broken in the defective structure). In terms of the Seitz notation of the International Tables for Crystallography [75], symmetry operations are expressed as $\square=(\boldsymbol{W}, \boldsymbol{w})$, where $\boldsymbol{W}$ represents the symmetry elements of rotation, reflection or inversion and $w$ represents translation. An analogous procedure for defects in bicrystals is the basis of the topological theory [1]. The symmetry of the CDP is the union of the two lattices space groups, extended by any color-reversing elements present interrelating them. Color-reversing symmetry elements are designated with a prime, e.g. $m$ ' instead of $m$ for a mirror plane. 

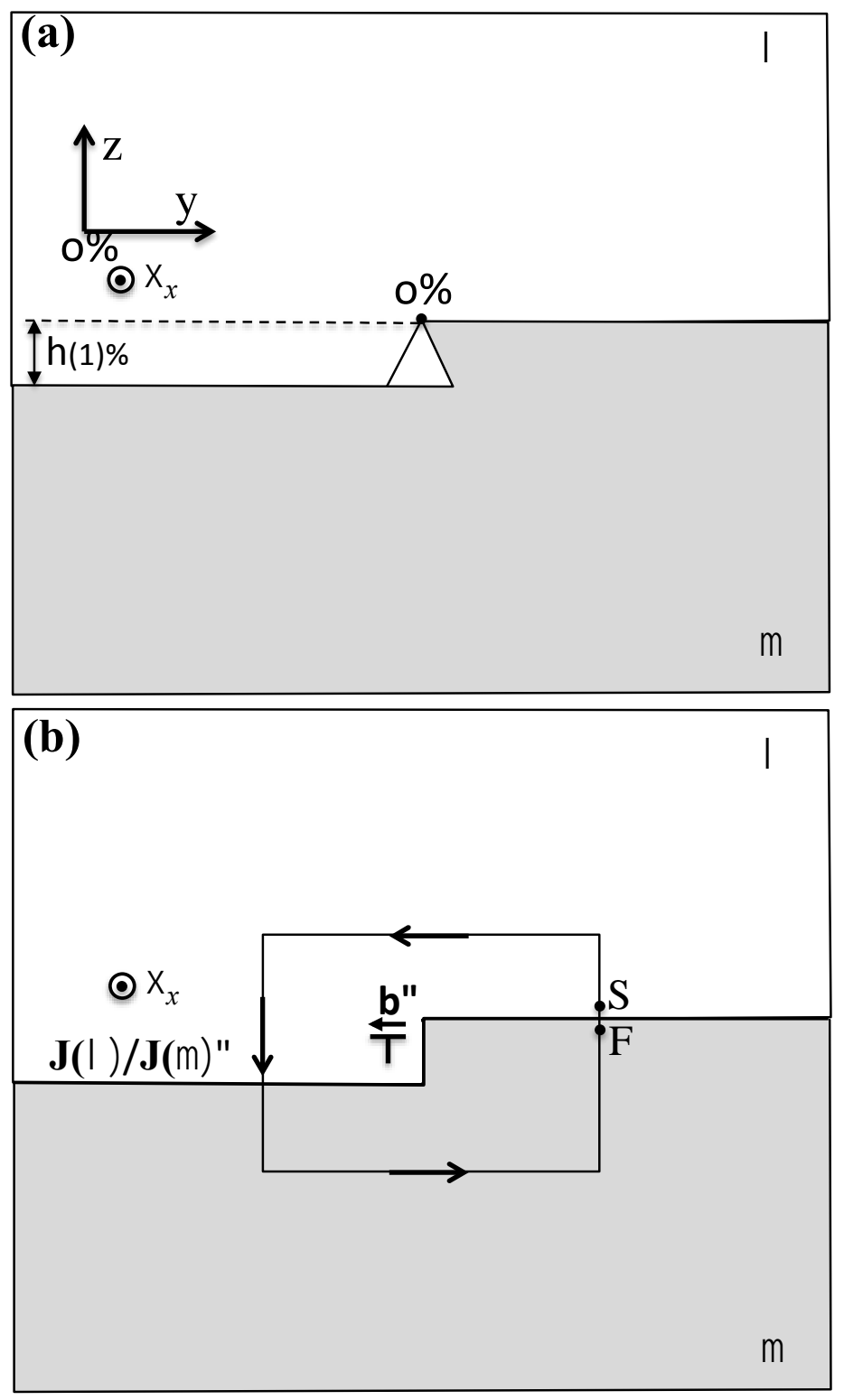

Figure A1. (a) Schematic illustration of matrix $\mu$ and product $\lambda$ crystals. The steps exhibited on each surface are incompatible, and lead to the formation of a disconnection. (b) The crystal surfaces are bonded to form coherent terraces bounding a disconnection. A circuit that is clockwise about the sense vector $\xi$ and closed in the distorted crystal is indicated.

For translational defects, displaced Volterra cuts, e.g., Figure 1 in Ref. [62] map directly to a CDC. The closure of the sides of the cut can be related to a circuit operator $\boldsymbol{C}$, representing in the $\boldsymbol{S} \boldsymbol{F}$ sense the set of operations that close the cut, creating identical 
structures on either side of the incompatibility [1]. An example is shown in Figure A1a. The corresponding closed circuit for a real defect is shown in Figure A1b.

Thus, provided a Volterra operation is carried out at the incompatible steps, the incompatibility becomes a perfect interfacial defect, characterized by the Volterra operation $\left(\boldsymbol{Q}_{\mathrm{ij}}, \boldsymbol{q}_{\mathrm{ij}}\right)$, the product of a white and a black symmetry operation. The Burgers vector of the resulting defect relates to translational symmetry and is given by

$$
\boldsymbol{b}=\boldsymbol{t}(\lambda)_{i}-\boldsymbol{P t}(\mu)_{j}
$$

where $\boldsymbol{P}$ is a coordinate transformation, re-expressing $\mathbf{t}(\mu)_{\mathrm{j}}$ in the $\lambda$-frame. Here, $\boldsymbol{t}(\lambda)_{\boldsymbol{i}}$ and $\boldsymbol{t}(\mu)_{j}$ are translation vectors in the dichromatic complex. In general, if the $\mu$ lattice were rigidly displaced from the $\lambda$ lattice by a vector $\boldsymbol{p}, \boldsymbol{P}$ in Eq. (A1) would be replaced by $(\boldsymbol{P}, \boldsymbol{p})$. This procedure is predictive, an a priori method, and is described in detail in $[1,2,35]$. Here, we emphasize the a posteriori method, deducing relevant defects by circuit mapping as described in Appendix B. These methods have physical connotations. In the first method, a cut is made in a perfect crystal and displacements needed to displace the cut and create a defect are determined. In the second method, displacements about a defect in a real crystal are mapped into a perfect reference space, as described in Appendix B, and the same displacements are deduced. Either method can be mapped either way, so the distinction is mainly in the methodology.

Here, we emphasize the circuit method. A detailed comparison of the two methods is given in Appendices A and B in [5], and not repeated here. In what follows, one could obtain the same result by either method. We outline the treatment for the case of twinning. Positions in the product in the ideal or real bicrystal are represented by $\mu$ to $\lambda$ displacement vectors determined in the CDC or CDP. These vectors are then described as in Appendix B. 


\section{Appendix B. Topological analysis of single defects}

\section{B.1. Burgers vector}

One starts with a closed circuit in the ideal bicrystal and maps it onto the CDC as shown in Figure B-1(a). Also, if one were to construct a closed circuit about the associated defect in a real bicrystal, it would also map into identical atom positions in the CDC. Thus the a posteriori circuit procedure gives identical results to the a priori topological theory of Pond [1]. We introduce the following conventions in order that the results agree with the equations in $[1,5]$ and to connect directly to the e-cells introduced in Sect. 2.

- Select the $\lambda$ phase as white and corresponding to the product (twin, martensite or diffusional plate) phase, with $\mu$ as the matrix.

- Select the unit interface normal vector $\mathbf{n}$ to point to white.

- Construct the circuit as right-handed relative to the sense vector $\xi$. (B1)

- Select the circuit start point $\mathbf{S}$ in the $\boldsymbol{\lambda}$ phase.

- Select $\mathbf{S}$ to start at the interface. This is not necessary, but makes the determination of imperfect defects simpler.

- For the extension to ideal bicrystals and e-cells, select the origin as an atom or center-of-symmetry site in the last $\lambda$ layer at the interface.

This convention is consistent with standard RH/FS Burgers circuits for dislocations in perfect crystals [62]. Only this convention consistently reproduces both Eq. (A1) and the same $\boldsymbol{b}$ in both methods. The final signs of $\boldsymbol{b}$ were correct in all previous TM papers, but the $\boldsymbol{t}$ vectors and white phase were not always assigned according to the convention. For reverse motion (de-twinning or reversion), $\lambda$ transforms to $\mu, \boldsymbol{b}$ is the same but shuffle $\boldsymbol{s}$ vectors are opposite in sign.

The procedure is as follows for the (111) $f c c$ twin in Figure B-1. In the real crystal, an observer traces out a closed clockwise circuit with respect to the defect linedirection, $\boldsymbol{\xi}$, here selected to point toward the reader, and hence the closed circuit is counterclockwise for the reader. The start point $\mathbf{S}$ is selected to always be in the $\lambda$ phase with the above convention. The closed circuit passes through "good" material in the real 
bicrystal, starting at $\mathbf{S}$, proceeding through the $\lambda$-crystal to $\mathbf{J}(\lambda)$, identical to $\mathbf{J}(\mu)$ when $\boldsymbol{p}$ is zero, and thence through the $\mu$-crystal back to $\mathbf{F}$, Figure A-1(b). When the vectors $\mathbf{S} / \mathbf{F}$ and $\mathbf{J}(\lambda) / \mathbf{J}(\mu)$ are mapped to an ideal bicrystal they are identical, and the observer is transported by equal and opposite displacements across the interface at these points. Hence, in combination, they contribute zero to the total circuit, can be disregarded in the CDC. The circuit could begin anywhere, but because the vectors SF and $\mathbf{J}(\lambda) \mathbf{J}(\mu)$ relate to shuffle vectors, the condition is imposed that the origin is selected so that $\mathbf{S}$ starts at the interface so that $\mathbf{S F}$ and $\mathbf{J}(\lambda) \mathbf{J}(\mu)$ span the dividing surface/interface. This is analogous to the procedure suggested by Frank [11, 250] for stacking faults. This circuit is then mapped into the CDP, where it fails to close, Figure B-1(a).

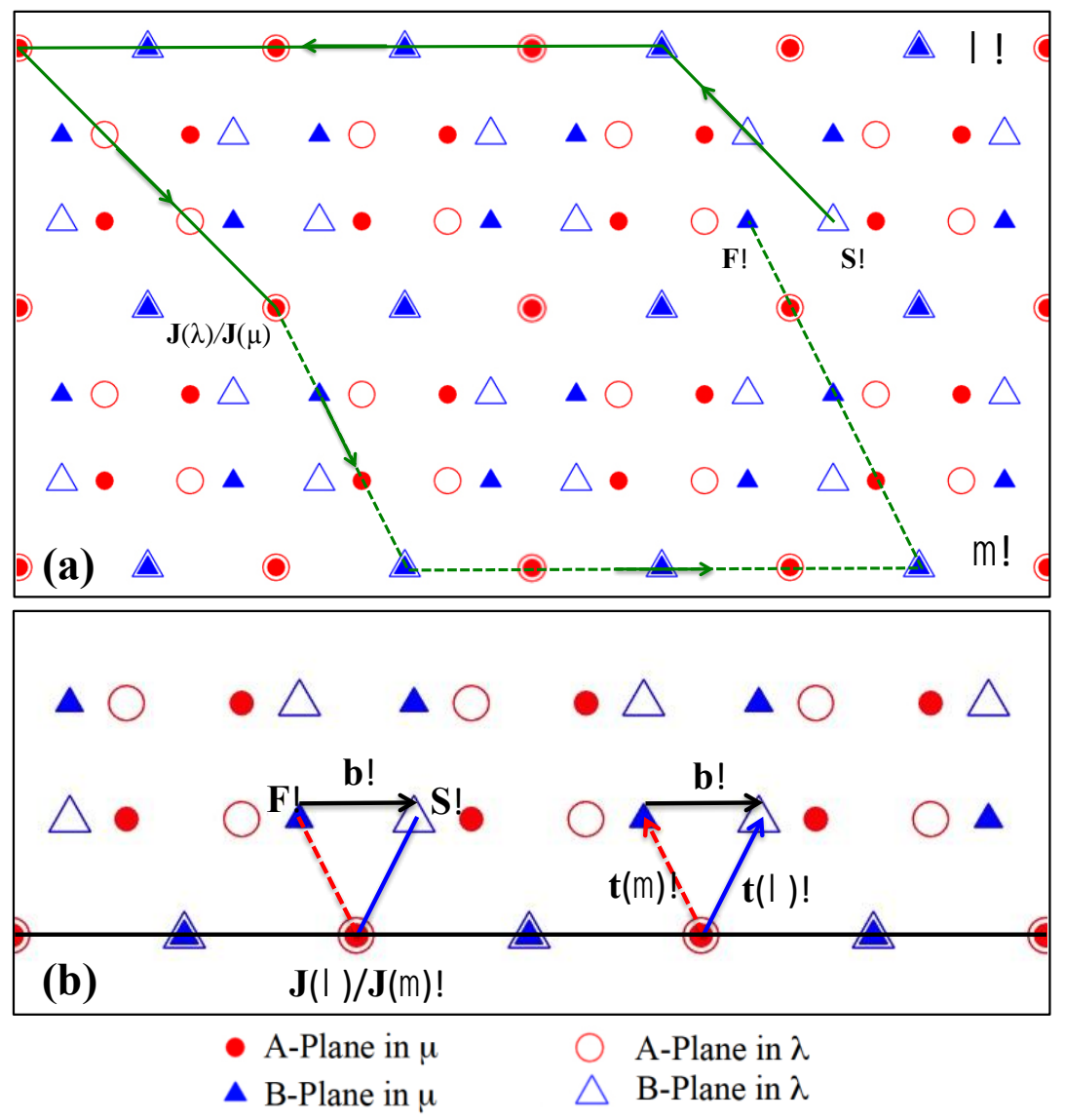

Figure B-1. (a) CDC (reference crystal) with mapped circuit. The sense vector $\xi$ points toward the reader. (b) Irreducible circuit.

One then shrinks the circuit. It is helpful to consider the cancellation of circuit elements in two stages. First, the cancelling terms $\mathbf{S F}$ and $\mathbf{J}(\lambda) \mathbf{J}(\mu)$ are neglected. The $\lambda$ - 
segment reduces to the vector $\mathbf{S} \mathbf{J}(\lambda)$, and the $\mu$-segment to $\mathbf{J}(\mu) \mathbf{F}$. In the Frank-Bilby formalism [38-40], the vector $\mathbf{J}(\lambda) \mathbf{S}$ is familiar as the probe vector $\mathbf{v}^{\text {sym }}$ (after mapping), while $\mathbf{S J}(\lambda)$ corresponds to the probe vector $\mathbf{v}$ in a mechanics descriptions, see [5]. By further cancellation of equal and opposite translations along the interface, i.e., by shrinking the circuits and transposing to the CDC as in Figure B-1(b), one obtains the irreducible description: the circuit in $\lambda$ reduces to $\mathbf{J}(\lambda) \mathbf{S}$ and that in $\mu$ to $\mathbf{J}(\mu) \mathbf{F}$. However, the vector $\mathbf{J}(\lambda) \mathbf{S}$ corresponds to $\mathbf{t}(\lambda)$ and the vector $\mathbf{J}(\mu) \mathbf{F}$ to $\mathbf{t}(\mu)$, The Burgers vector is given by the closure vector FS. Thus

$$
\boldsymbol{b}=\mathbf{J}(\lambda) \mathbf{S}+\mathbf{J}(\mu) \mathbf{F}=\mathbf{J}(\lambda) \mathbf{S}-\mathbf{J}(\mu) \mathbf{F}=\mathbf{t}(\lambda)-\mathbf{t}(\mu)
$$

The subscripts of Eq. (A1) are dropped because we are treating unit disconnections. We emphasize that only the CDP is needed to determine $\boldsymbol{b}$ via the $\boldsymbol{t}$ vectors.

\section{B.2. Steps}

In general, the heights of the two surface steps shown in Figure A-1(a) differ as $h(\lambda)$ and $h(\mu)$. The one with smaller magnitude, designated as the "overlap" then establishes the step height, $h$. In general, with differing terrace plane spacings, m planes of the matrix and q planes of the product, where $\mathrm{m}$ and $\mathrm{q}$ are integers, contribute to the step height and we characterize the step as $h(\mathrm{~m}, \mathrm{q})$ and select its length as the shorter of the $\mu$ or $\lambda$ steps. This designation is necessary for some examples of facets in the text. For disconnections in type I twinning, the step heights of $\mu$ and $\lambda$ are identical and the designation is written as $h(\mathrm{~m})$. One can ascribe a sign convention, defining steps as being positive or negative with respect to a given interface. The steps are categorized as positive when the normal components of the vectors, $\boldsymbol{n} \bullet \mathbf{t}(\lambda)$ and $\boldsymbol{n} \bullet \mathbf{t}(\mu)$, are positive, and negative for the converse, as shown in Figure B-2. The signs of both $\boldsymbol{b}$ and $h(\mathrm{~m}, \mathrm{q})$ or $\mathrm{h}(\mathrm{m})$ change on reversing the line-sense of a disconnection. Here, $\boldsymbol{b}$ is zero, and the CDC is required in order to represent the shuffles in forming or moving a step. Figure B-2 represents a single twin. The four disconnections each differ, but the $\mathbf{t}$ vectors are all plus/minus versions of one another so subscripts are dropped. It could represent four different twins, in which case all vector quantities could vary in different quadrants and the latter would be separated by domain walls. This more general situation, described in 
Figure 22 in Ref. [4] along with symmetry restrictions, rarely occurs for twins. In the general sense, Figure B-2 shows how the $\mathrm{t}_{\mathrm{z}}$ components of $\mathbf{t}$ vectors relate to $\boldsymbol{n}$ and the sign of the step in accord with convention B-1.

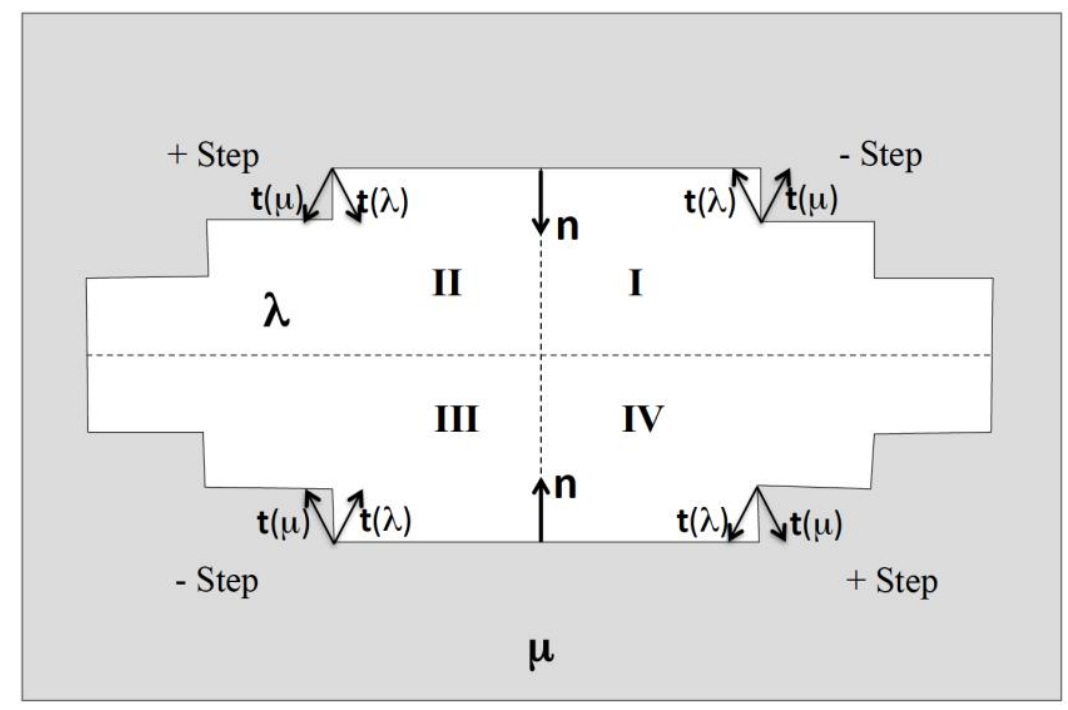

Figure B-2. Schematic view of possible disconnections and associated properties.

B.3. Imperfect and domain defects.

For an imperfect defect, the appropriate vectors are no longer translation vectors in the CDC. However possible imperfect Burgers vectors still relate to an expression resembling the above expression.

$$
\boldsymbol{b}=\boldsymbol{q}(\lambda)-\boldsymbol{q}(\mu)
$$

The vectors $\boldsymbol{q}$, required to define imperfect dislocations or dislocation components of disconnections, are not translation vectors in a CDP but can be represented in a CDC or translated CDC (TCDC), although they may not connect atom sites. The vectors were defined as $\boldsymbol{v}$ in [1], but are changed to $\boldsymbol{q}$ here to avoid confusion with other uses for $\boldsymbol{v}$.

A domain line separates two degenerate terraces that are rotated with respect to one another. The defect Burgers vector for parallel terraces is given by Eq. (B4) in ref. [1].

$$
\boldsymbol{b}=\boldsymbol{w}(\lambda)-\boldsymbol{w}(\mu)
$$




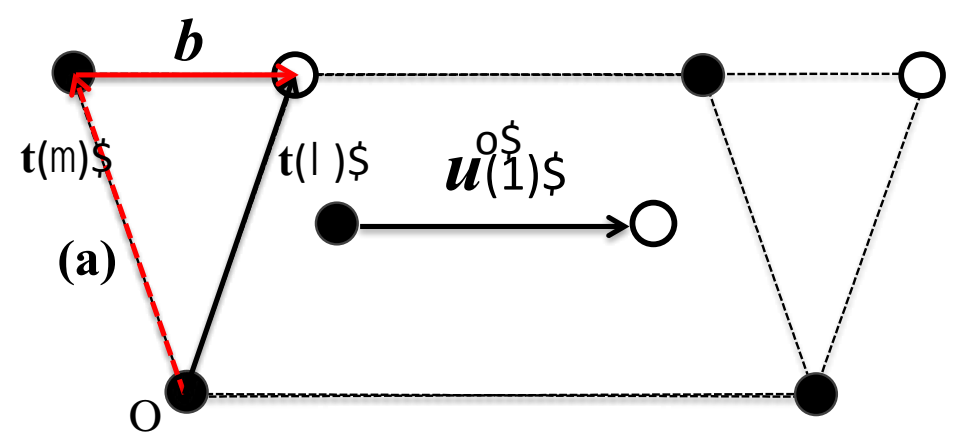

(b)

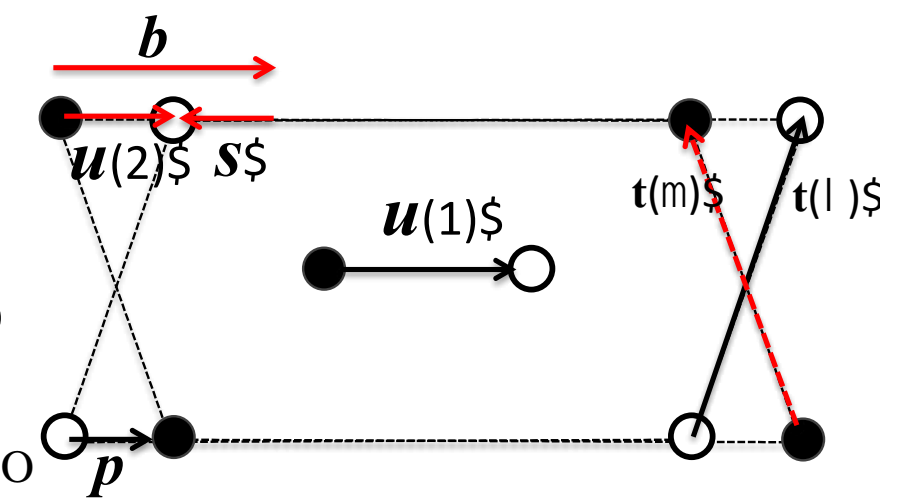

(c)

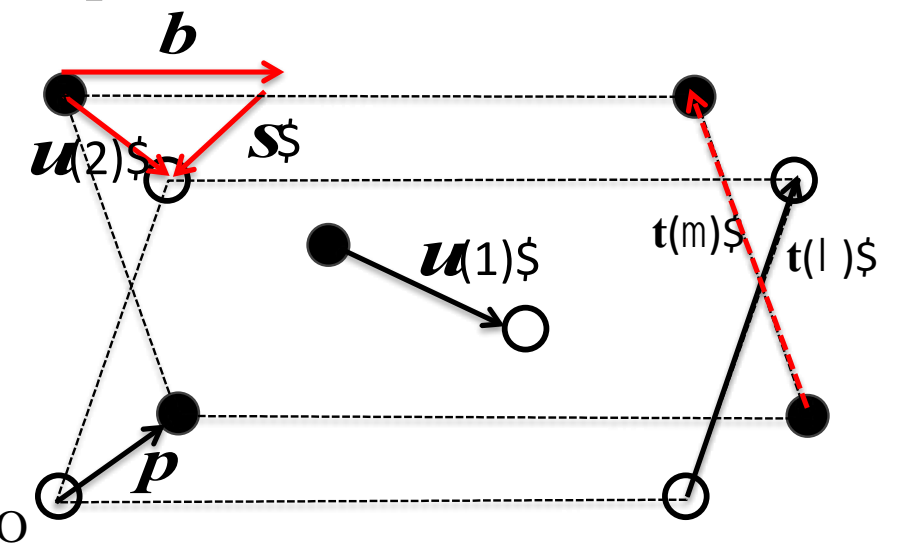

Figure B-3. E-cell for monoclinic twin. (a) With $\boldsymbol{p}=0$. (b) and (c) With $\boldsymbol{p}$ finite.

\section{B.4. Shuffles}

Shuffle vectors represented in e-cells also do not usually correspond to translation vectors in the CDC but correspond to position vectors $\boldsymbol{u}^{\mathbf{0}}$, where the superscript 0 indicates that $\boldsymbol{p}=0$. Position vectors are in general connected by displacement vectors $\boldsymbol{u}$ that can comprise $\boldsymbol{b}, \boldsymbol{s}, \boldsymbol{p}$ or $\boldsymbol{f}$, see below, or a combination of two or more of these. Thus, shuffle vectors are 


$$
\boldsymbol{s}=\boldsymbol{u}^{\mathbf{0}}(\lambda)-\boldsymbol{u}^{\mathbf{0}}(\mu)
$$

For display of shuffles, the CDC or an e-cell is again required. Figure B-3 shows an e-cell for a hypothetical case, where the crystal is simple monoclinic with a basis of 2 . Figure B-3(a) shows the shuffle when $\boldsymbol{p}$, the rigid shift of the $\mu$ lattice relative to $\lambda$, is zero, and Figure B-3(b) shows the shuffle when $\boldsymbol{p}$ is non-zero with $x$ and $y$ components, and Figure B-3(c) with $p_{z}$ components added. For coherent twin boundaries, $p_{z}$ is usually very small. As illustrated, when $\boldsymbol{p}$ is finite, all shuffle vectors are modified to

$$
\boldsymbol{s}=\boldsymbol{u}(\lambda)-\boldsymbol{u}(\mu)=\boldsymbol{u}^{0}(\lambda)-\boldsymbol{u}^{0}(\mu)-\boldsymbol{p}
$$

In particular, note that the top atoms acquire a shuffle $-\boldsymbol{p}$ although the $\boldsymbol{u}^{\mathbf{0}}$ vectors are zero. The operator $\boldsymbol{P}$ in Eq. (A1) is replaced by $(\boldsymbol{P}, \boldsymbol{p})$, the CDC is shifted by $\boldsymbol{p}$ to a TCDC and the e-cell is accordingly displaced as shown in Figure B-3. Hence, the e-cell is useful in depicting displacement vectors and relevant defect vector quantities directly.

\section{B.5. Other defects.}

Other defects such as disclinations with associated Frank vectors $\boldsymbol{f}$ are described in the text.

\section{Appendix C. Twin notation}

In the classical designation shown in Figure C-1 [32, 251, 252], twins with rational values of $K_{1}$ and $\boldsymbol{\eta}_{2}$ are designated Type I, and those with rational values of $K_{2}$ and $\boldsymbol{\eta}_{1}$ are Type II: all four parameters have rational indices for compound twins. The basic requirement for a compound twin is that the plane normal to $\boldsymbol{\eta}_{1}$ is a low-index mirror plane. As discussed in Sect. 11, true compound twins are rare and many cases so designated actually deviate by a small rotation from the true case. This has been recognized in $[32,58]$. They define a twin by the shape change arising from a region formed by the plane strain deformation with only deviatoric components of the strain tensor. In general this would lead to irrational, non-reproducible interfaces. For the TM, with terraces, a modifier for Type $\mathrm{I}$ is that the twin $K_{1}$ plane be a mirror plane in the matrix. For Type II, $\boldsymbol{\eta}_{2}$ must be normal to a mirror plane. This more general description fits well with mechanistic treatments such as the Topological Model [3-5], which are atomistic models unknown when the theory of twinning was initiated. Indeed, this could 
be broadened to a twin being a region formed by the plastic distortion (strain plus rotation) of a region in the matrix, modified as above.

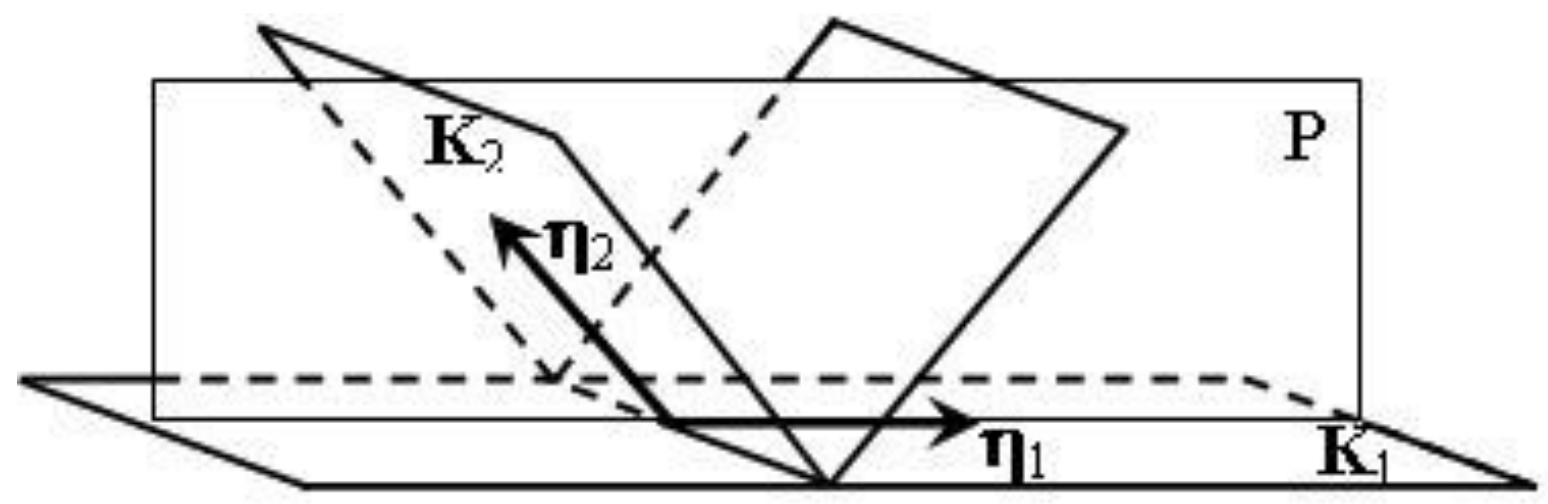

Figure C-1. Classical twinning elements.

Nevertheless, one can associate Type I twinning with the mechanism of twinning dislocations/disconnections (TDs) propagating on a terrace plane. In the TM, the key feature is that twinning is produced by defect motion. In principle, a twin in $f c c$, for example, could be produced by two sets of screw dislocations accumulating and producing a $60^{\circ}$ twist boundary. However this can be ruled out because of the repulsive pinning interactions of intersections of dislocations from the two sets. Thus we are left with the mechanism wherein the twinning is produced by the motion of TDs. The plastic distortion associated with TD motion is an engineering shear, $\gamma_{y z}$, determined by the Burgers (shear) vector, $b_{y}$ on the terrace (glide) plane normal to $\mathrm{z}$, and comprises a tensor strain and a rotation,

$$
\gamma_{y z}=\left(b_{y} / d_{z}\right)
$$

Where $d_{z}$ is the interplanar spacing of the terrace (glide) plane. This engineering shear, with its contained rotation, makes a connection of the TM with both the Phenomenological Theory of Martensite Crystallography (PTMC) [23-25] theory for phase transformation and to mechanics descriptions of the macroscopic strains associated with twinning [209, 217, 253].

When a disconnection has a unique $\boldsymbol{b}$, a translation vector of the lattice must lie in the undistorted $K_{2}$ plane and hence $\eta_{2}$ is rational: type I. If dislocations with several $\boldsymbol{b}$ are 
present, either $K_{2}$ or $\boldsymbol{\eta}_{2}$ or both can be irrational. If after twinning, the extent of each terrace twin plane is large, then the terrace regions near the interface resemble the classical picture. The twinning distortion (shear plus rotation) takes place entirely within the matrix being twinned and the interface is unaffected. The undistorted plane is symmetrically displaced as in the classical twinning picture, Figure A-1. If there is a $K_{2}$ plane with a common zone axis with $K_{1}$ the undistorted plane is $K_{2}$ and the twin is compound. However, for two crystals in twin orientation, there can be many habit planes other than $K_{1}$, and many can be irrational [67]. Indeed, cases where three or four of these parameters can be irrational have been discussed $[32,58,108]$. As one example, if the defects present on a twin or interphase interface are pure steps, there is perfect twin orientation but the mean interface is irrational. One other requirement for the TD case, which follows from simple geometry, is that the TD Burgers vector cannot be that of a perfect dislocation the matrix: it must be a perfect interfacial defect, whose Burgers vector corresponds to that of a partial in a single crystal. Of course the vector is imperfect in either the matrix or the twin, consistent with the classical designation.

In the other TM mechanism, TDs with a Burgers vector $\boldsymbol{b}$ parallel to $\boldsymbol{\eta}_{2}$ glide on a plane $K_{2}$ slanted with respect to the final twin plane, producing a distortion that again has a rotation component. Hence $K_{2}$ is rational. As discussed in Sect. 11, a key feature of the TM is that the rotation must be partitioned to the two crystals. Hence, the Burgers vector component normal to the twinning plane must also be partitioned. Since $\boldsymbol{b}$ is rational the unpartitioned component in the twin plane must be rational, i.e., $\boldsymbol{\eta}_{1}$ would be rational: Type II. After partitioning, the component of $\boldsymbol{b}$ in the $K_{l}$ plane and hence $\boldsymbol{\eta}_{1}$ would be irrational. In Sect. 11, we show that this "type II process" in some special cases leads to a rational $K_{l}$ but in others it leads to an irrational $K_{1}$.

More generally, the classical scheme is expressed in terms of rational/irrational parameters, and this doesn't map into symmetry descriptions directly. For example, in centro-symmetric crystals, the composition plane of compound twins is expected to be a mirror plane classically. However, in cases like the $(11 \overline{2} 2)$ twin, the mirror is broken when the non-symmorphic symmetry of $h c p$ is taken into account, leading to the 
admissibility of two interface structures separated by a domain defect [1]. This mirror breaking is also likely to occur for tilt walls.

Finally we note that for $f c c$ compound twins with $K_{\mathrm{I}}$ as a (111) plane, the conjugate plane is also a $\{111\}$ plane. This is rare and for (103) $f c c$ twins, the conjugate twinning plane can be $\{103\}$, but there are many other conjugate planes with different crystallographic normals, e.g., $\{501\}$. This is true in other crystal systems, such as $h c p$. In many low-symmetry crystals, there are no compound twins.

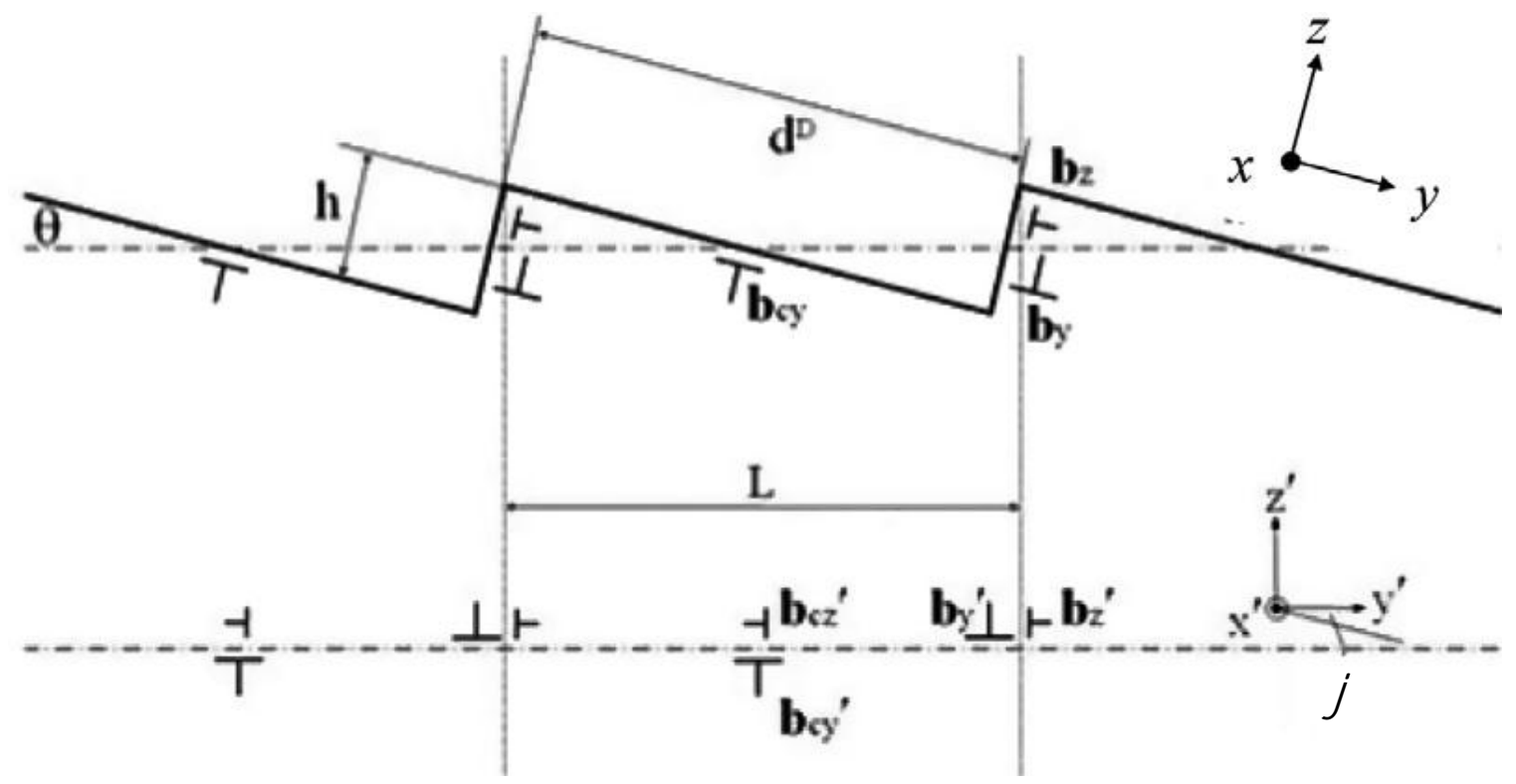

Figure D-1. Disconnection array at an interface indicated by dashed line. Burgers vector components shown in interface coordinates in lower figure.

\section{Appendix D. Arrays of defects}

With the properties of a single defect described as in Appendix B, the remaining part of the TM is to describe arrays of defects as discussed in detail in [4, 7]. The two main features of the TM are depicted in Figures D-1 and D-2. For a single dislocation or disconnection, the distortion field has a rotation component but it is negligible. However, for an array of disconnections, the rotations sum to give a significant tilt as in Figure D-1, true also for a pure dislocation array. Following classical dislocation theory dating back to Read [52], this rotation $\varphi$ must be partitioned to the two grains in order that no coherency strains arise in the interface. With partitioning, the components $\boldsymbol{b}_{z}{ }^{\prime}$ produce a 
proper tilt wall. This important feature is not incorporated in the PTMC theory for phase transformations and accounts for the differences between the PTMC and the TM. If the spacing $\mathrm{L}$ is properly adjusted, all misfit strains are relieved. This is indicated in Figure D-1 by the cancellation of the coherency field, indicated by an equivalent Burgers vector $\boldsymbol{b}_{c}^{\prime}$, by the field of the disconnection Burgers vectors $\boldsymbol{b}^{\prime}$, expressed in interface coordinates $x_{i}{ }^{\prime}$. The latter would be directly represented in a rotated CDC (RCDC) where the $\lambda$ and $\mu$ lattices are rotated by $\pm \theta$ relative to the coherent plane in the CDC.

Figure D-2 illustrates a deformation twin as an array of disconnections: only the Burgers vectors are shown. Once the disconnection properties are defined, the stress field can be determined from a non-planar pileup configuration [254]. There the disconnections are in an elastic equilibrium array with respect to one another but there is a large uncompensated incompatibility stress at the tip. There are $\boldsymbol{b}_{\mathbf{z}}$ components normal to the mean interface, so there are also rotational incompatibilities. At the very tip, the pileup is blunted [124] into a short discontinuous pileup [62], as verified in $\mathrm{Mg}$ in a HRTEM study [125]. If the discrete pileup is smeared into a continuous infinitesimal array, the ellipsoidal representation as a stressed inclusion [255], useful in a macroscopic mechanics description [217], appears.

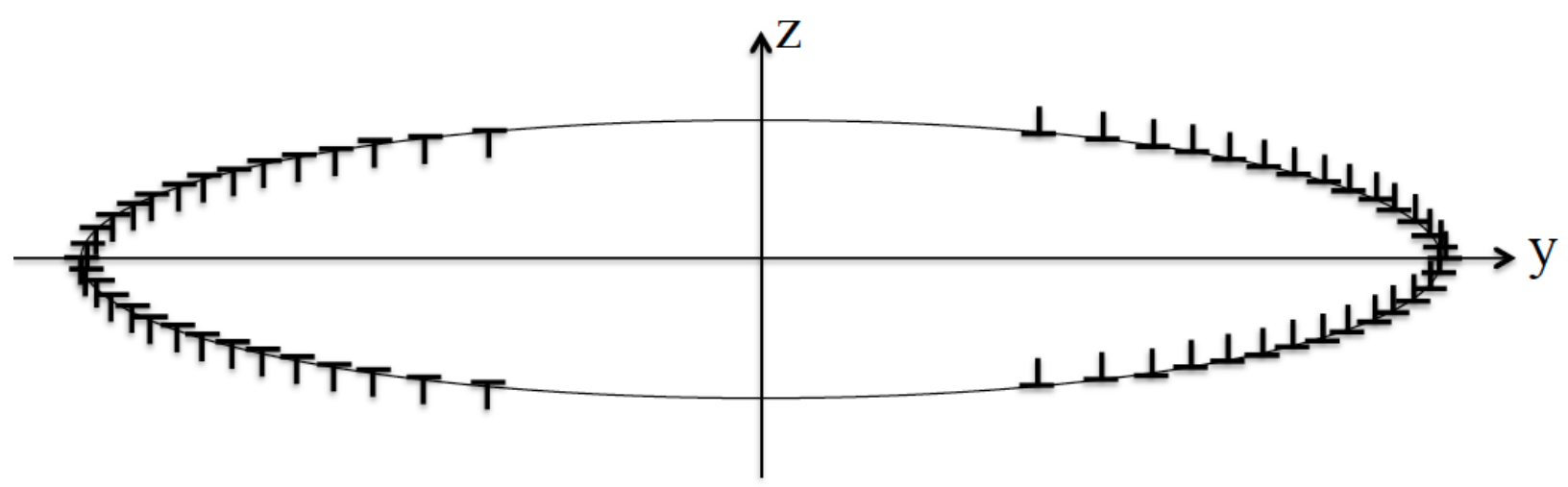

Figure D-2. Burgers vector components of disconnections produce long-range stresses shown for a deformation twin. If these are smeared into a continuous infinitesimal dislocation array, the ellipsoidal representation used in mechanics descriptions is formed. 


\section{Appendix E. List of abbreviations}

CDC: commensurate (coherent) dichromatic complex.

CDP: commensurate (coherent) dichromatic pattern.

COS: center-of-symmetry

DFT: density functional theory.

DSC: displacement shift complete (lattice).

EAM: embedded atom method.

EBSD: electron back scattering diffraction.

FFT: fast Fourier transform.

HRTEM: high-resolution transmission electron microscopy

MD: molecular dynamics.

NDP: natural dichromatic pattern.

$\mathrm{P} / \mathrm{B}$ : prism/basal (interface plane).

PTMC: phenomenological theory of martensite crystallography.

RCDC: rotated commensurate (coherent) dichromatic complex.

RCDP: rotated commensurate (coherent) dichromatic pattern.

TD: twinning disconnection.

TEM: transmission electron microscopy.

TM: topological model.

VPSC: visco-plastic self-consistent model. 